\title{
Inflation forecasting using the New Keynesian Phillips Curve with a time- varying trend
}

Article

Accepted Version

Creative Commons: Attribution-Noncommercial-No Derivative Works 4.0

McKnight, S., Mihailov, A. and Rumler, F. (2020) Inflation forecasting using the New Keynesian Phillips Curve with a time-varying trend. Economic Modelling, 87. pp. 383-393. ISSN 0264-9993 doi:

https://doi.org/10.1016/j.econmod.2019.08.011 Available at https://centaur.reading.ac.uk/85596/

It is advisable to refer to the publisher's version if you intend to cite from the work. See Guidance on citing.

To link to this article DOI: http://dx.doi.org/10.1016/j.econmod.2019.08.011

Publisher: Elsevier

All outputs in CentAUR are protected by Intellectual Property Rights law, including copyright law. Copyright and IPR is retained by the creators or other copyright holders. Terms and conditions for use of this material are defined in the End User Agreement.

www.reading.ac.uk/centaur 
Central Archive at the University of Reading

Reading's research outputs online 


\title{
Inflation Forecasting Using the New Keynesian Phillips Curve with a Time-Varying Trend
}

\author{
Stephen McKnight* Alexander Mihailov ${ }^{\dagger}$ Fabio Rumler ${ }^{\ddagger}$ \\ Accepted (pre-print) version at Economic Modelling on 14 August $2019^{\S}$
}

\begin{abstract}
Does theory aid inflation forecasting? To address this question, we develop a novel forecasting procedure based upon a New Keynesian Phillips Curve that incorporates time-varying trend inflation, to capture shifts in central bank preferences and monetary policy frameworks. We generate theory-implied predictions for both the trend and cyclical components of inflation, and recombine them to obtain an overall inflation forecast. Using quarterly data for the Euro Area and the United States that cover almost half a century, we compare our inflation forecasting procedure against the most popular time series models. We find that our theorybased forecasts outperform these benchmarks that previous studies found difficult to beat. Our results are shown to be robust to structural breaks, geographic areas, and variants of the econometric specification. Our findings suggest that the skepticism concerning the use of theory in forecasting is unwarranted, and theory should continue to play an important role in policymaking.
\end{abstract}

Keywords: inflation forecasting; predictive accuracy; New Keynesian Phillips Curve; time-varying trend

JEL classification: C53; D43; E31; E37; F41; F47

\footnotetext{
*Centro de Estudios Económicos, El Colegio de México, Carretera Picacho Ajusco No. 20, Col. Ampliación Fuentes del Pedregal, Delegación Tlalpan, Mexico City, C.P. 10740, Mexico; mcknight@colmex.mx

${ }^{\dagger}$ Corresponding author: Department of Economics, University of Reading, Whiteknights, Reading RG6 6AA, United Kingdom; a.mihailov@reading.ac.uk

${ }_{\ddagger}^{\ddagger}$ Oesterreichische Nationalbank, Economic Analysis Division, Otto Wagner Platz 3, A-1090 Vienna, Austria; fabio.rumler@oenb.at

$\S$ We would like to thank the editor, Sushanta Mallick, and two anonymous referees of this journal for helpful comments and suggestions. We are also grateful for the input of our co-author in earlier drafts, Kerry Patterson, who retired. We acknowledge feedback from participants at the workshop on Inflation Modelling and Forecasting: Recent Approaches (University of Reading, 2012), the 2013 EEA-ESEM meeting, the 2013 LACEA-LAMES meeting, and seminars at the University of the West of England and Webster University Vienna. Mihailov gratefully acknowledges funding from a British Academy Small Research Grant (Reference SG100243) at the start-up phase of this project. The usual disclaimer applies.
} 


\section{HIGHLIGHTS}

- A challenge in inflation forecasting has been the skepticism regarding theory-based models.

- We derive a New Keynesian Phillips Curve with a time-varying trend.

- We then incorporate it into a novel procedure to forecast inflation.

- These modifications improve forecasting performance in the Euro Area and the United States.

- Our approach can complement other methods used at central banks over policyrelevant horizons. 


\section{Introduction}

"Without resort to theory [...] conclusions relevant to the guidance of policies cannot be drawn." Koopmans (1947: p. 167)

Given its policy importance, there exists a substantial literature on inflation dynamics and the associated problem of inflation forecasting. ${ }^{1}$. While one strand of the literature is broadly agnostic about the underlying macroeconomic model that generates the inflation path, ${ }^{2}$ another strand has adopted a structural approach, with explicit links to macroeconomic models with microeconomic foundations. A key component of the microfounded approach to inflation dynamics is the estimation of the so-called New Keynesian Phillips Curve (NKPC), an aggregate inflation equation arising in dynamic stochastic general equilibrium (DSGE) models with price adjustment frictions. ${ }^{3}$ However, the inflation forecasts generated from single-equation (also known as limited information) methods involving the NKPC have not been able to compete against simple agnostic time-series models. This paper proposes two important modifications to help improve the predictive performance of the NKPC. First, in order to capture shifts in central bank preferences and monetary policy frameworks, we introduce variation in trend inflation. Second, our NKPC with a time-varying trend is incorporated into a novel inflation forecasting procedure that is able to generate reliable theory-based inflation predictions at policy-relevant horizons. The novelty is that theory-implied laws of motion inform the modelling, and subsequent forecasting, of the trend and cyclical components of observed inflation.

There is growing skepticism in the existing literature with regard to the benefits of using the NKPC approach to forecast inflation. Empirical findings from a number of studies have shown that Phillips curve-based inflation forecasts perform poorly in 'pseudoout-of-sample' predictive evaluation against a good univariate benchmark (e.g., Stock and Watson, 2009). Notably, Atkeson and Ohanian (2001), henceforth AO, found that since 1984 Phillips curve forecasts for inflation in the United States (US) were inferior to a naïve forecast of 12-month inflation by its average rate over the previous 12 months. The AO finding has proven difficult to overturn in inflation forecasting (e.g., Stock and Watson,

\footnotetext{
${ }^{1}$ For comprehensive surveys, see Stock and Watson (1999, 2003, 2007, 2009); Edge and Gürkaynak (2010); Rossi and Sekhposyan (2010, 2014); Ball and Mazumder (2011); Faust and Wright (2013); Dotsey et al. (2018).

${ }^{2}$ This approach applies variants of popular time-series analysis techniques that impose minimal theoretical restrictions, e.g., Engle and Granger (1987), Lütkepohl (1987), Harvey (1989) and Johansen (1996). For a completely agnostic approach - known as Singular Spectrum Analysis - centred on the learning from pure data structures (without any a priori economic theory modeling), see, e.g., Golyandina et al. (2001), Hassani et al. (2009) and Patterson et al. (2010).

${ }^{3}$ Since Galí and Gertler (1999), the most popular approach to estimate the NKPC is via limitedinformation single-equation methods, which use the generalized method of moments (GMM) proposed by Hansen (1982) to operationalize the expectational terms under the rational expectations hypothesis. See, e.g., Galí et al. (2001, 2003, 2005); Sbordone (2002, 2005); Leith and Malley (2007); Rumler (2007); Mihailov et al. (2011 a, b); King and Watson (2012).
} 
2007, 2009; Faust and Wright, 2013). Faust and Wright (2013) evaluate the predictive performance of a set of 16 commonly applied inflation forecasting methods and three judgemental forecasts of inflation based on private sector surveys and the Greenbook. They conclude that judgmental forecasts are remarkably hard to beat, and also find that very simple methods, which limit or avoid parameter estimation, tend to predict inflation relatively well. Again, the AO 'pseudo' or 'average' random walk (RW) forecast comes out among the best-performing forecasting methods in terms of predictive accuracy, not only in inflation forecasts for the US, but also for Canada, Germany, Japan, and the United Kingdom.

However, the existing literature that forecasts inflation using the NKPC has ignored time-varying trend inflation from the analysis, by assuming that inflation is either zero or constant in the steady state. This paper addresses this gap in the forecasting literature. While several papers have incorporated variations in trend inflation to model inflation dynamics, e.g., Adolfson et al. (2007) in conducting a Bayesian estimation for Sweden using a small open-economy DSGE model, Cogley and Sbordone (2008) with a single-equation estimation focus on US data, and Yazgan and Yilmazkuday (2007) and Yilmazkuday (2007) in estimating monetary policy rules, this is the first study to examine their implications for inflation forecasting using the limited information NKPC approach.

As in Adolfson et al. (2007), we introduce drifting trend inflation by assuming price indexation to both last-period actual inflation and current-period trend inflation. ${ }^{4}$ From an empirical and policymaking perspective, such an indexation scheme by the private sector is important as it captures both inflation persistence and, notably, shifts in monetary policy preferences and inflation targets. Since central bank mandates and policymaking frameworks evolve over time in response to changes in the macroeconomic and institutional environment, there should be potential gains, especially in medium- and longerhorizon inflation forecasting, from capturing such shifts. Moreover, these assumptions result in inflation dynamics that are broadly consistent with the time-series properties of our data set.

Armed with our NKPC that incorporates shifts in trend inflation, we propose a novel 'time-varying trend New Keynesian Phillips Curve (TVT-NKPC)' procedure to forecast inflation. It highlights the predictive value of allowing for the pervasive drifting trends in observed macrovariables derived from the microfoundations of price setting and the resulting aggregate NKPC equation. Our inflation forecasting procedure consists of first applying a one-sided Hodrick-Prescott (1997) filter to separate trend from cyclical components in each fixed-length rolling window or augmenting-length recursive window for re-estimation and prediction that resembles real-time forecasting in our pseudo-out-of-

\footnotetext{
${ }^{4}$ Cogley and Sbordone (2008) incorporate drifting trend inflation by assuming price indexation to lagged inflation only. They show, using Bayesian time-varying coefficient VAR methods, that a purely forward-looking version of the NKPC fits US quarterly data well for the period 1960:1-2003:4.
} 
sample exercise. ${ }^{5}$ Then, the TVT-NKPC equation for the cyclical component of inflation generates forecasts for this component via a corresponding theory-implied auxiliary vector autoregression (VAR). ${ }^{6}$ Following Galí and Gertler (1999), unobservable real marginal cost (RMC) is proxied by real unit labor cost (RULC). Alternatively, we also construct a calibrated proxy for RMC based on the open-economy monetary model of McKnight and Mihailov (2015). Our TVT-NKPC theory also generates predictions for trend inflation, which are employed in the forecasting of the trend component of inflation as a stochastic $\mathrm{AR}(1)$ process that incorporates a unit root. We build on the predictive accuracy comparisons in Stock and Watson (2007) and Faust and Wright (2013), by selecting the agnostic AO inflation forecast and driftless RW as the main benchmarks to evaluate the predictive performance of our TVT-NKPC inflation forecasting procedure. ${ }^{7}$

Predictive accuracy is assessed pseudo-out-of-sample via the commonly adopted criterion of root mean squared forecast error at the policy-relevant horizons of 1, 4 ('short run'), 8, 12 ('medium run'), 16 and 20 ('long run') quarters using quarterly time series for the Euro Area (EA) and the US that cover almost half a century, 1970:1-2015:4 (184 quarters), and a forecasting evaluation period that constitutes, roughly, one-third of the sample, 2000:1-2015:4 (64 quarters). In contrast to the skepticism of the existing literature, the results from the inflation predictions generated from our theory-based NKPC method are positive. We find that the TVT-NKPC forecasting procedure outperforms the conventional random walk benchmark at all horizons, up to a margin of 20-25 percentage points in the medium- and long-run inflation forecasts (significantly at 1 and 8 quarters in the EA and US data and also at 16 and 20 quarters in the EA case). Moreover, it also outperforms quantitatively, by 10-20 percentage points beyond the short run, the AO benchmark that previous studies have found difficult to beat (significantly at the medium run in the US case). Overall, our findings offer encouragement on the potential of the TVT-NKPC to generate reliable theory-based forecasts of inflation.

Our results are shown to be robust across structural breaks, involving in particular the periods of the "great moderation", the global financial crisis, and the zero lower bound of quantitative easing. Our forecasting improvements are also robust for both the EA and the US, and for two alternative econometric specifications: fixed rolling window versus augmenting window. Further, we demonstrate robustness regardless of the proxy used

\footnotetext{
${ }^{5}$ The rolling-window re-estimation, in particular, handles gradual (and possibly unknown) structural change, as argued in Bauwens et al. (2015), and is implemented in many inflation forecasting studies (see, e.g., Kascha and Ravazzolo, 2010).

${ }^{6}$ As far as the cyclical component of inflation is concerned, our approach is related to some extent to the so-called 'semi-structural' forecasting methods found in the studies of Rumler and Valderrama (2010), Liu and Jansen (2011), Kichian and Rumler (2014) and Posch and Rumler (2015). However, this literature ignores time-varying trend inflation and assumes agnostic time-series models for real unit labor cost as the single driving variable in the NKPC equation. The semi-structural approach has had only nonsystematic, partial success in specific countries and at specific horizons relative to conventional benchmarks.

${ }^{7}$ The driftless RW has been the conventional forecasting benchmark since Meese and Rogoff (1983).
} 
for unobservable real marginal costs.

Our findings suggest that drifting trends, in effect derived carefully from microfoundations, play an important role in the predictive performance of limited information NKPC models. Consequently, theory-implied forecasts are at least as good, and at many time horizons better, than agnostic statistical predictions of inflation. Being so, our forecasting method can complement other approaches used to predict inflation at central banks over policy-relevant horizons. In particular, our method generates inflation forecasts that are comparable even to high-dimensional DSGE systems, but remains parsimonious and much less demanding in terms of data and assumptions.

The paper is organized as follows. The next section derives the TVT-NKPC and justifies theoretically the concept of fundamental inflation and the law of motion of trend inflation employed in the forecasts. Section 3 discusses our data and empirical implementation, while section 4 reports and interprets our predictive evaluation results. The final section concludes. Supplementary material is available online that includes Appendices A-C. ${ }^{8}$

\section{Theoretical Framework}

In this section, we briefly summarize our TVT-NKPC inflation forecasting procedure, leaving the details in Appendices B.1 and B.2.

We assume that there is full indexation of non-optimized prices for any firm $i, P_{t}(i)$, to both last-period actual (gross) inflation $\Pi_{t-1}$ with weight $0 \leq \rho \leq 1$ (as in Cogley and Sbordone, 2008) and current-period time-varying trend (gross) inflation $\bar{\Pi}_{t}$ with weight $1-\rho$ (as in Adolfson et al., 2007):

$$
P_{t}(i)=\Pi_{t-1}^{\rho} \bar{\Pi}_{t}^{1-\rho} P_{t-1}(i)
$$

In what follows, $\widehat{x}_{t}$ denotes either stationary log-deviations of variables $\widetilde{x}_{t}$ from their drifting trend value $\bar{x}_{t}$ or stationary log gross growth rates. Equation (1) implies, under Calvo (1983) price setting, the following generalized NKPC:

$$
\widehat{\Pi}_{t}-\rho \widehat{\Pi}_{t-1}+\rho \widehat{g}_{t}^{\bar{\Pi}}=\frac{(1-\alpha)\left(1-\alpha \beta g^{\Lambda} g^{Y}\right)}{\alpha(1+\epsilon \omega)} \widehat{M C}_{t}+\beta g^{\Lambda} g^{Y} E_{t}\left[\widehat{\Pi}_{t+1}-\rho \widehat{\Pi}_{t}+\rho \widehat{g}_{t+1}\right]
$$

where $\widehat{\Pi}_{t}$ denotes the cyclical component of period $t$ inflation, $\widehat{M C}_{t}$ is the cyclical component of period $t$ average real marginal cost, and $\widehat{g}_{t}^{\bar{\Pi}}$ is the growth rate of the time-varying inflation trend in $t$ relative to $t-1$. The parameters $\alpha, \epsilon, \omega, \beta, g^{Y}$ and $g^{\Lambda}$ capture,

\footnotetext{
${ }^{8}$ Appendix A contains a full description of our data set, with relevant sources and definitions; Appendix B provides additional derivations and technical detail; Appendix C presents and briefly discusses additional results (mostly illustrated in figures and tables to which we sometimes refer to later on).
} 
respectively, the Calvo probability of not being able to optimize own price in any period $t$, the elasticity of substitution across differentiated products, the degree of strategic complementarity, the deterministic discount factor, and the growth rates of output and of the marginal utility of wealth of firm shareholders, both evaluated as constants.

We transform the generalized NKPC equation (2) into a form that is more suitable for forecasting inflation. We assume that time-varying trend inflation follows an $\operatorname{AR}(1)$ process: ${ }^{9}$

$$
\widehat{g}_{t}^{\bar{\pi}}=\theta \widehat{g}_{t-1}^{\bar{\pi}}+\varepsilon_{\widehat{g}^{\bar{\pi}}, t},
$$

where $0<\theta<1$ is the persistence parameter (to be re-estimated in recursive subsamples in our forecasting procedure) and $\varepsilon_{\widehat{g}^{\pi}, t} \equiv \ln \varepsilon_{g^{\bar{\pi}}, t} \sim$ i.i.d. $\left(0, \sigma_{\widehat{g}^{\pi}}^{2}\right)$. Using (3), we can re-write (2) in quasi-first difference form as:

$$
\widehat{\pi}_{t}-\rho \widehat{\pi}_{t-1}=\gamma\left[E_{t} \widehat{\pi}_{t+1}-\rho \widehat{\pi}_{t}\right]+\kappa \widehat{m c}_{t}+\rho(\theta \gamma-1) \widehat{g}_{t}^{\bar{\pi}}
$$

where

$$
\kappa \equiv \frac{(1-\alpha)\left(1-\alpha \beta g^{\Lambda} g^{Y}\right)}{\alpha(1+\epsilon \omega)} \quad \text { and } \quad \gamma \equiv \beta g^{\Lambda} g^{Y}
$$

As discussed by Cogley and Sbordone (2008), estimates of $\rho$ tend to cluster around the point estimate of $0.2{ }^{10}$ Therefore, we calibrate $\rho=0.2$ in (4) for the purposes of re-estimating the other two reduced-form parameters in the TVT-NKPC in quasi-first differences, $\kappa$ and $\gamma$.

The generalized TVT-NKPC given by (4) can be reduced to two common special cases typically found in the literature. If $\rho=1$, indexation is only to last-period actual inflation, and this leads to the TVT-NKPC estimated in Cogley and Sbordone (2008). For example, assuming a zero net inflation steady state (where trend inflation is constant and so $\widehat{g}_{t}^{\bar{\pi}}=0$; and similarly $g^{\Lambda}=g^{Y}=1$ so that $\gamma=\beta$ ), the NKPC given in (4) reduces to the well-known 'hybrid' NKPC, containing a backward-looking term, $\widehat{\pi}_{t-1}$, that arises from this indexation:

$$
\widehat{\pi}_{t}=\frac{1}{1+\beta} \widehat{\pi}_{t-1}+\frac{\beta}{1+\beta} E_{t} \widehat{\pi}_{t+1}+\kappa \widehat{m c}_{t}
$$

If $\rho=0$, indexation is only to current trend inflation. For example, once again assuming a zero net inflation steady state, (4) reduces to the standard 'pure' NKPC:

$$
\widehat{\pi}_{t}=\beta E_{t} \widehat{\pi}_{t+1}+\kappa \widehat{m c}_{t} .
$$

\footnotetext{
${ }^{9}$ The $\mathrm{AR}(1)$ trend-growth assumption is consistent with the time-series properties of our data set summarized in section 3 further below; see also Table 1 in Appendix C (online).

${ }^{10}$ Most of these estimates relate to the partial indexation to past inflation only. However, Adolfson et al. (2007) estimate $\rho$ with Bayesian methods assuming full indexation to both past actual inflation and current trend inflation, as we do, and report a posterior mode of 0.212 (with standard deviation of 0.066 ) and a posterior mean of 0.217 .
} 
Comparing the hybrid (5) and pure (6) versions of the NKPC against the generalized TVT-NKPC (4) shows that current inflation $\widehat{\pi}_{t}$ depends importantly on an additional driver, namely, current-period innovations to trend inflation, $\widehat{g}_{t}^{\bar{\pi}}$ (affecting the intercept of the TVT-NKPC). Furthermore, trend growth in output, $g^{Y}$, and in the marginal utility of wealth of firm owners, $g^{\Lambda}$ - by entering the definitions of the reduced-form parameters of the TVT-NKPC, $\gamma$ and $\kappa$ - affects both its intercept and slope.

We employ two alternative proxies for real marginal costs (RMC): (i) the standard RULC proxy of Galí and Gertler (1999); and (ii) a proxy using the open-economy monetary model of McKnight and Mihailov (2015), which assumes nonseparable utility preferences for consumption and real money balances. A log-linear approximation around the time-varying trend assumed here yields the following expression for the cyclical component of RMC:

$$
\widehat{m c}_{t}=\bar{\omega} \widehat{Y}_{t}+\sigma \widehat{C}_{t}-\chi \widehat{m}_{t}+(1-a) \widehat{S}_{t}
$$

where $C_{t}, m_{t}$, and $S_{t}$ denote consumption, real money balances, and the (Home economy) terms of trade, respectively. For the parameters given in $(7), \bar{\omega}>0$ is the inverse of the Frisch labor supply elasticity, $\sigma>0$ is the coefficient of relative risk aversion, $\chi \geq 0$ is the degree of nonseparability of real money balances, and $0<a \leq 1$ is the degree of home bias in production. ${ }^{11}$ The structural parameters of (7) are calibrated using the values justified by McKnight and Mihailov (2015). Namely, we set $\bar{\omega}=0.47, \sigma=0.16, \chi=0.02$ and $a=0.85$. Consequently, given the four observable time series for $Y_{t}, C_{t}, m_{t}$ and $S_{t}$, we can obtain a proxy for the unobservable (cyclical component of) real marginal cost implied by the equilibrium conditions of a microfounded open-economy monetary model.

The law of motion for drifting trend inflation implies a straightforward one-step ahead forecast (for $t$-indexed variables assumed observed or estimated):

$$
E_{t}\left[\bar{\Pi}_{t+1}\right] \equiv \bar{\Pi}_{t+1 \mid t}=g_{t}^{\bar{\Pi}} \bar{\Pi}_{t}
$$

We then use this forecast in generating iterative forecasts for any horizon $h=1, \ldots, 20$ quarters for the trend component of inflation after re-estimating (recursively) $g_{t}^{\bar{\Pi}}$ from the data. The $h$-step ahead forecast based on this stochastic $\operatorname{AR}(1)$ trend dynamics becomes

$$
\bar{\Pi}_{t+h \mid t}=\left(g_{t}^{\bar{\Pi}}\right)^{h} \bar{\Pi}_{t}, \text { for } h=1, \ldots, 20 .
$$

This is the forecasting procedure for time-varying trend inflation we implement after reestimation of $g_{t}^{\bar{\Pi}}$ at every prediction origin, and then add up to the corresponding forecast for the cyclical component of inflation generated according to the TVT-NKPC, which we

\footnotetext{
${ }^{11}$ As discussed by Woodford (2003), in popular cashless economies (i.e., $\chi=0$ ), a policymaker does not face a trade-off between inflation and output stabilization. However, with $\chi>0$ money enters (7) as a negative 'cost-push' shock.
} 
describe next.

Evaluating the implied 'fundamental inflation' measure that arises in equation (4) requires multiperiod forecasts of the forcing variables. These are generated in our case from a 3-variate VAR with 4 lags (denoted as $3 \mathrm{VAR}(4)$ ) of dimension 12:

$$
\underset{(1 \times 12)}{\widehat{Z}_{t}^{\prime}}=\left[\widehat{m c}_{t-j_{p}}, \widehat{g}_{t-j_{p}}^{\bar{\pi}}, \widehat{\pi}_{t-j_{p}}-\rho \widehat{\pi}_{t-\left(j_{p}+1\right)}\right]^{\prime}, \quad j_{p}=\{0,1,2,3\}
$$

Note that $0<\kappa, \rho(\theta \gamma-1)<1$, and that $\widehat{Z}_{t+h \mid t}=\mathbf{A}^{h} \widehat{Z}_{t}$, where $\widehat{Z}_{t}$ denotes the vector entering the companion form matrix $\mathbf{A}$ of the companion $3 \operatorname{VAR}(1)$ system and $h$ the horizon of the forecasts with origin $t$. We can therefore apply the summation formula for infinite geometric sequences to the stationary forward-looking solution of the (re-) estimated TVT-NKPC equation (4) and obtain fundamental inflation in period $t$ :

$$
\widehat{\pi}_{t}-\rho \widehat{\pi}_{t-1}=\kappa \underset{(1 \times 12)}{e_{1}^{\prime}}\left(\mathbf{I}_{12}-\gamma \underset{(12 \times 12)}{\mathbf{A}}\right)^{-1} \underset{(12 \times 1)}{\widehat{Z}_{t}}+\rho(\theta \gamma-1) \underset{(1 \times 12)}{e_{2}^{\prime}}\left(\mathbf{I}_{12}-\gamma \underset{(12 \times 12)}{\mathbf{A}}\right)^{-1} \underset{(12 \times 1)}{\widehat{Z}_{t}} .
$$

In (10), the selection vector $e_{1}^{\prime}$ (with 1 as its first element and 0's elsewhere) extracts the forecast for $\widehat{m c}_{t+h}$ (first row of $\mathbf{A}$ ) while the selection vector $e_{2}^{\prime}$ (with 1 as its second element and 0's elsewhere) that for $\widehat{g}_{t+h}^{\bar{\pi}}$ (second row of $\mathbf{A}$ ).

In order to generate forecasts for the cyclical component of inflation, $\widehat{\pi}_{t+h \mid t}$, conditional on information at time $t$, lead expression (10) by one period and note that the 1-periodahead forecast of $\widehat{Z}_{t}$ is $\widehat{Z}_{t+1 \mid t}=\mathbf{A} \widehat{Z}_{t}$. Hence the 1-step-ahead forecast of the cyclical component of inflation, $\widehat{\pi}_{t+1 \mid t}$, based on the model-consistent concept of fundamental inflation implied by our TVT-NKPC theory, is given in terms of current variables as follows: ${ }^{12}$

$$
E_{t}\left[\widehat{\pi}_{t+1}\right] \equiv \widehat{\pi}_{t+1 \mid t}=\rho \widehat{\pi}_{t}+\kappa e_{1}^{\prime}(\mathbf{I}-\gamma \mathbf{A})^{-1} \mathbf{A} \widehat{Z}_{t}+\rho(\theta \gamma-1) e_{2}^{\prime}(\mathbf{I}-\gamma \mathbf{A})^{-1} \mathbf{A} \widehat{Z}_{t}
$$

Iterating this cyclical inflation forecast forward, we can construct an $h$-step-ahead forecast of cyclical inflation. The outcome is the following general forecasting equation:

$$
\widehat{\pi}_{t+h \mid t}=\rho^{h} \widehat{\pi}_{t}+\kappa e_{1}^{\prime}(\mathbf{I}-\gamma \mathbf{A})^{-1} \sum_{i=1}^{h} \rho^{i-1} \mathbf{A}^{i} \widehat{Z}_{t}+\rho(\theta \gamma-1) e_{2}^{\prime}(\mathbf{I}-\gamma \mathbf{A})^{-1} \sum_{i=1}^{h} \rho^{i-1} \mathbf{A}^{i} \widehat{Z}_{t},
$$

which is used to generate the forecasts for the cyclical component of inflation. ${ }^{13}$

\footnotetext{
${ }^{12}$ For simplicity, we suppress the explicit notation for the matrix dimensions.

${ }^{13}$ The auxiliary VAR we use in forecasting can be viewed as nesting the NKPC in other setups (e.g, Posch and Rumler, 2015), where this is accounted for by jointly estimating the parameters of the VAR and the NKPC. In the present paper, however, the process for marginal cost is much more complicated, which is why the NKPC is not nested in the VAR.
} 


\section{Empirical Implementation}

In the present section we discuss our empirical implementation of the proposed theory of inflation dynamics to forecasting inflation in the EA and the US.

We use quarterly data for the EA and the US over almost half a century, from 1970:1 to 2015:4 (184 quarters), and compare forecast accuracy in a pseudo-out-of-sample evaluation period of approximately the last third of our full sample, 2000:1-2015:4 (64 quarters), as is common practice. Appendix A (online) provides details of the data sources, definitions and mnemonics.

Our quarterly inflation measure is defined in a standard way (in annualized \% terms),

$$
\pi_{t} \equiv 400 \times \ln \frac{P_{t}}{P_{t-1}}
$$

where $P_{t}$ is the quarterly GDP-deflator price index. Our preference here for the GDPdeflator price index as a measure of the aggregate price level in the EA and the US follows the inflation forecasting literature, notably Stock and Watson (2007) and Faust and Wright (2013). ${ }^{14}$

We follow Faust and Wright (2013) in performing iterated multistep forecasts, and not direct forecasts. ${ }^{15}$ We also follow Faust and Wright (2013) in forecasting single-quarter inflation rates, and not cumulative inflation rates over a particular future horizon as done, e.g., in Stock and Watson (2007). The reason is that the former approach allows to judge in a more straightforward way how the forecast horizon may affect the predictability of inflation: if cumulating predicted inflation rates instead, the shorter- and longer-run forecast accuracy will be conflated over the duration of the respective forecast horizon.

[Table 1 about here]

Table 1 summarizes the models of inflation dynamics included in our predictive accuracy comparisons. As in Stock and Watson (2007), among others, we implement the forecasts in two variants, using recursively re-estimated (i) fixed-length rolling sample window and (ii) augmenting-length sample window. In addition, and also as a robustness check, the predictive performance of the TVT-NKPC forecasting procedure is examined in two other variants, depending on the proxy for unobservable real marginal cost (RMC), either (i) a monetary open economy (MOE) RMC proxy constructed from four observable variables as implied by equation (7) above, or (ii) the standard real unit labor cost (RULC) proxy for RMC. These TVT-NKPC forecasts are then compared against the

\footnotetext{
${ }^{14}$ As discussed in Clark and Doh (2014), this is a broader aggregate price measure than the alternative consumer price index (CPI) measure of inflation. While these two common inflation measures do not generally stray too far apart, they do not follow precisely the same dynamics. CPI inflation tends to be more volatile than GDP-deflator inflation, especially in the US data (see figures 2 and 3 in Appendix C).

${ }^{15}$ See Appendix B.3 (online) for definitions and further detail on iterated versus direct forecasts.
} 
forecasts from two typical univariate statistical specifications for inflation as (alternative and agnostic) benchmarks, (i) the conventional driftless random walk and (ii) the widely used AO pseudo random walk. Under the random walk without drift univariate benchmark, which predicts the same inflation for any quarter at any horizon $h$ as that observed in the most recent quarter, the inflation forecast can be written as (assuming again that $t$-indexed variables are observed)

$$
\pi_{t+h \mid t}=\pi_{t}
$$

For the second univariate benchmark we employ the AO pseudo random walk, which is essentially a random walk forecast designed for the horizon of 12 months, with Stock and Watson (2007) adapting it to quarterly data and extending the AO forecast to other horizons. ${ }^{16}$ The iterated version of the AO forecast, adjusted to our quarterly frequency, can then be written as

$$
\pi_{t+h \mid t} \equiv \frac{1}{4}\left(\pi_{t}+\pi_{t-1}+\pi_{t-2}+\pi_{t-3}\right)
$$

That is, the adjusted AO model forecasts the 4-quarter-ahead inflation and, by extension, the inflation in any earlier or future quarter, $h(=1, \ldots, 20$ in our case), to be the same as the average of the latest four quarters of observed inflation.

It is important to emphasize as well the congruence of the theoretical choices introduced earlier with the corresponding empirical implementation of our novel forecasting procedure. More specifically, the theory-based trend and cyclical inflation components implied by our derivation of the TVT-NKPC are also consistent with the stationarity properties of both the EA and US data, as evidenced by the formal test results reported in Table 1 in Appendix $\mathrm{C}$ (online) and as illustrated visually in Figures 1-4, to which we return with some discussion further down. Accordingly - in a 'double', theory-informed and data-supported, justification - trend inflation is forecast using the univariate stochastic AR(1) trend model defined in (3), while cyclical inflation is forecast by the auxiliary $3 \mathrm{VAR}(4)$ model embodied in (12) as an empirical implementation of (4). ${ }^{17}$

In line with the literature, we report and compare accuracy for pseudo-out-of-sample predictions in terms of the Theil U-statistic, defined as the ratio of the root mean squared forecast error (MSFE) of the (theory-based) model of inflation dynamics (in the numerator of Theil's ratio) relative to that of the respective benchmark, RW or AO in our case, (in the denominator) for the policy-relevant horizons of 1, 4, 8, 12, 16 and 20 quarters. The modified Diebold-Mariano (1995) test (MDM), proposed by Harvey et al. (1997) to correct for small-sample bias, checks for the statistical significance of the difference in

\footnotetext{
${ }^{16}$ Atkeson and Ohanian (2001) proposed a naïve model of US inflation dynamics initially designed for the monthly frequency that has nevertheless performed very well at horizons of 1 and 2 years in the US data.

${ }^{17}$ The final instrument set and lags in the TVT-NKPC GMM re-estimation was selected with view to the Hansen (1982) J-test statistic for the validity of the overidentifying restrictions.
} 
predictive accuracy between pairs of non-nested model forecasts. ${ }^{18}$ We focus on the onesided version of the p-value for the MDM test because we are interested here primarily in the improvement - not the deterioration - in predictive accuracy over the respective benchmark (i.e., Theil's U ratios lower than 1) that arises from the application of our TVT-NKPC forecasting procedure.

Figure 1 depicts the EA (blue) and US (red) quarterly GDP-deflator inflation at annualized \% rate in our sample (1970:1-2015:4), where the shaded area corresponds to our forecasting evaluation period (2000:1-2015:4). The patterns of inflation dynamics in the EA and the US display some similarities as well as some differences. The high inflation of the 1970s, due to the global oil shocks, and the subsequent disinflation of the 1980s is a common feature, yet during these first two decades in our sample EA inflation was higher than US inflation (and, unsurprisingly, quite heterogeneous within the area). The 'great moderation' in the world economy, usually identified to have begun in the mid-1980s for the US, but somewhat later for the EA, after the European exchange-rate mechanism crisis of 1992:3-1993:2, and to have ended with the recent global financial crisis (GFC) of 2007:3-2009:4, is evident in inflation dynamics in both the EA and US data. Then, in the last 15 years of the sample, with the ECB now responsible for EA monetary policy, inflation has been low and relatively stable: lower and less volatile compared to the US, and almost reversing itself into deflation near the end of the GFC.

\section{[Figure 1 around here]}

We have kept the turbulent GFC quarters together with the preceding 'great moderation' low inflation subperiod and the subsequent 'deflation scare' subperiod, where a number of economies have been operating at the zero lower bound of nominal interest rates, in part with the goal to ensure a relatively long rolling window; in part we also wanted to subject our forecasting procedure to a more challenging empirical test. More precisely, the fixed-window length in the rolling estimation, which is equivalent to the initial estimation sample in the augmenting-window recursive estimation, is 113 effective quarters, with the first quarter of 2000 being the horizon of our first 1-step-ahead inflation prediction over the forecasting evaluation period.

The results from the stationarity tests we performed in our full sample are reported in Table 1 of Appendix $\mathrm{C}$ (online). Inflation is conclusively found to be nonstationary in both the EA and US data; that is, by both the Augmented Dickey-Fuller (ADF) test with a null of unit root (with constant included as well as excluded) ${ }^{19}$ at all conventional significance levels and the Kwiatkowski-Phillips-Schmidt-Shin (KPSS) test with a null

\footnotetext{
${ }^{18}$ For a detailed discussion of the rationale and advantages of using the MDM test in forecast accuracy comparisons, see Faust and Wright (2013), Clark and McCracken (2013), and Clark and Doh (2014).

${ }^{19}$ See Dickey and Fuller (1979) and Said and Dickey (1984), as well as Schwarz (1978) and MacKinnon (1996).
} 
of stationarity (with constant included) ${ }^{20}$ at the $5 \%$ and $10 \%$ significance levels (not at the $1 \%$ level). When a linear trend is also included, in addition to the constant in the ADF and KPSS specifications, this trend comes out statistically significant and negative for both the EA and the US, which is clearly seen too by inspection of Figure 1 . The formal statistical tests here regarding (non)stationarity are important in that their findings support the TVT-NKPC forecasting procedure. The methodology proposed here relies on first separating a time-varying inflation trend from the inflation cycle, before the prediction exercise for observed inflation is then undertaken by components (with potentially differing or diverging dynamic or stationarity properties).

[Figures 2 and 3 about here]

Figures 2 and 3 illustrate these trend and cycle components, respectively, of EA and US quarterly GDP-deflator inflation at annualized rate. ${ }^{21}$ Again, checking Table 1 in Appendix C (online), the ADF and KPSS tests are conclusive with regard to establishing the stationarity of the inflation cycle around the drifting inflation trend in both the EA and the US at all conventional levels of statistical significance. The KPSS test is also conclusive regarding the nonstationarity (in our sample) of trend inflation in both the EA and the US, whereas the ADF test confirms this finding at all conventional significance levels only for the US but not for the EA (at the $10 \%$ level).

[Figure 4 about here]

Figure 4 depicts the EA (blue) and US (red) change in quarterly (log-) GDP-deflator trend inflation at annualized \% rate, as another major determinant of inflation dynamics around a time-varying trend according to the generalized NKPC equation (4). It is found stationary (in our sample) for both the EA and the US at all conventional significance levels by the KPSS test. In addition, for both the EA and the US, it is found stationary at the $5 \%$ and $10 \%$ levels by the ADF test too, but nonstationary at the $1 \%$ level. These findings are broadly consistent with the theoretical law of motion imposed in equation (3).

Finally, figures 4 and 5 in Appendix $\mathrm{C}$ (online) depict the cyclical component of the EA (blue) and US (red) quarterly MOE and RULC proxies, respectively, for the (log-) real marginal cost at annualized \% rate, as a major determinant of inflation dynamics according to the TVT-NKPC equation (4). The findings in Table 1 in Appendix C (online) are convincing once again in establishing stationarity of the cyclical component of both these proxies for RMC around their drifting underlying trends in both the EA

\footnotetext{
${ }^{20}$ See Kwiatkowski et al. (1992), as well as Bartlett (1950) and Andrews (1991).

${ }^{21}$ In all figures illustrating trend or cycle of a time series, these two components have been separated for the whole sample by applying a two-sided Hodrick-Prescott (1997) filter. See also Figure 1 in Appendix $\mathrm{C}$ (online) for the robsutness of our Hodrick-Prescott trend inflation measure in the US case.
} 
and the US data at all conventional levels of statistical significance. One could also notice that the MOE RMC measure and the RULC RMC alternative do not exhibit quite the same patterns of dynamics and volatility in our data set, and therefore may not be equally useful in the forecasting exercise at its various horizons.

The stationarity test results summarized above therefore support the TVT-NKPC forecasting procedure. The cyclical component of inflation, or rather its empirically constructed analogue in our sample, is stationary because all of its component drivers in equation (4) are stationary; hence the projection for the cyclical component for inflation extracted via the companion form of the theory-implied 3VAR(4), as in (12), is justified and in line with the required stationarity for the implemented forecasting iterations at any horizon ahead.

\section{Results on Predictive Accuracy}

In this section we present the key results from evaluating the predictive accuracy of our TVT-NKPC inflation forecasting procedure. Additional estimation and forecasting results, as further robustness checks, are presented in Appendix C (online). We begin by discussing our findings with regard to the EA data, before moving to the US case.

\subsection{Forecasting EA Inflation}

Our main results with regard to the EA, reported in Table 2, can be summarized as follows.

\section{[Table 2 about here]}

First, comparing the root mean square forecast errors in panel A of the table, it can be seen that the RW benchmark results in the worst predictive accuracy, that is the largest root MSFE, across all six horizons, relative to the AO benchmark forecast and all four variants of the TVT-NKPC procedure. This finding reveals that our theorybased forecasting procedure is able to extract predictive content from EA inflation data that helps to improve forecasting compared to the RW benchmark of nonpredictability. Moreover, at the medium (8 and 12 quarters) and longer (16 and 20 quarters) horizons, the TVT-NKPC procedure in all its four variants, that is, no matter the re-estimation of either a fixed- or augmenting-length recursive window or the choice of proxy used for $\mathrm{RMC}$, achieves the most accurate prediction, beating in terms of root MSFE even the AO forecast. With bold fonts denoting the best forecast by horizon, one can see that for the EA data the RULC RMC rolling-window forecast dominates, winning at 8, 12 and 16 quarters, while the RULC RMC augmenting-window comes most accurate at the longest 
horizon of 20 quarters. However, at the two shorter-run horizons of 1 and 4 quarters the AO benchmark predicts EA inflation with the smallest root MSFE. ${ }^{22}$

We next consider the difference in predictive accuracy comparing the TVT-NKPC forecasts with the RW benchmark, reported in panel B of Table 2. The TVT-NKPC forecasts beat the RW forecast at all six horizons, with a gain ranging from $22.4 \%$ (statistically significant at horizon of 1 quarter, where the benefit of the MOE RMC proxy in the augmenting-window variant is illustrated) to $26.8 \%$ (statistically significant at horizon of 16 quarters, where the advantage of the RULC RMC proxy in the rolling-window variant becomes evident). More generally, our TVT-NKPC forecasts are significantly more accurate than the RW forecast at all horizons beyond the immediate short run of 1 quarter (where they are statistically worse) except 12 quarters (yet still numerically better by $19.1 \%$ ) and 4 quarters (numerically better by $1.3 \%$ ).

Third, to further judge whether the best variant of the TVT-NKPC forecast is more accurate than the AO forecast in a statistically significant way, we compute Theil's U ratio using the root MSFE of the AO forecast as the denominator and the (one-sided) MDM statistics for this benchmark. Panel $\mathrm{C}$ of Table 2 reports that these two compared forecasts are not statistically distinguishable at all horizons. Of note, however, at the medium (8 and 12 quarters) and longer (16 and 20 quarters) horizons, the TVT-NKPC forecast (in all its four variants) outperforms the $\mathrm{AO}$ forecast, by a numerical margin of up to $9.9 \%$.

Comparing the four variants of the TVT-NKPC forecasts, we see from Table 2 that re-estimating a fixed- versus augmenting-length recursive window of the data and using the RULC versus MOE RMC proxy tends to perform better, especially at horizons of 12 and 16 quarters. Yet the use of the MOE RMC proxy in its augmenting-window implementation brings a considerable advantage in coming very close to the best forecast at the immediate horizon of 1 quarter and at the longest horizon of 20 quarters.

\subsection{Forecasting US Inflation}

Turning to the US data, our main results reported in Table 3 do not change the essence of the conclusions summarized in section 4.1 for the EA data; there are however some interesting differences and nuances worth mentioning, as follows.

[Table 3 about here]

First, now the MOE-RMC fixed rolling window variant comes out as the most accurate forecast (i.e., lowest root MSFE, in bold), winning at two horizons, 1 and 16 quarters.

\footnotetext{
${ }^{22}$ Figures 14 (short run), 16 (medium run) and 18 (long run) in Appendix C provide a visual illustration for the best-performing variant of our TVT-NKPC forecasts against actual inflation in the EA.
} 
The AO forecast is best only at 4 quarters, whereas the RW forecast is not that much far off overall. ${ }^{23}$

Next, in panel B of Table 3 we see that in the US case the TVT-NKPC forecast in its MOE-RMC fixed rolling window version is statistically more accurate than the RW forecast at horizon of 1 quarter, whereas the RULC-RMC variant (in both windows, fixed and augmenting) is statistically dominating the RW at 8 quarters. At the remaining four prediction horizons the best TVT-NKPC variant is not statistically distinguishable from the RW forecast, but is numerically much more accurate, with gains from $7.6 \%$ at 4 quarters to $21 \%$ (MOE-RMC in augmenting window, again) at 20 quarters.

Third, panel $\mathrm{C}$ of Table 3 reveals that now, in the US data, the best variant of our TVT-NKPC forecast dominates the AO forecast in a statistically significant way at the medium run of 8 and 12 quarters. It also dominates numerically, by an important margin, the $\mathrm{AO}$ forecast at the long run of 16 (18.8\%) and 20 (8.6\%) quarters, remaining indistinguishable statistically at the short run of 1 and 4 quarters from the AO benchmark.

Finally, looking across the four variants of implementing the TVT-NKPC forecasts, we see from Table 3, and somewhat consistent with the analogous conclusion in the EA data, that none of these variants really dominates the remaining ones across more than 1-2 of the 6 forecast horizons examined.

\subsection{Policy-Relevant Insights from Our EA and US Inflation Forecasts}

Overall, for the EA we can conclude that the TVT-NKPC procedure significantly outperforms the RW benchmark at all horizons except 4 and 12 quarters ahead; however, even when the statistical significance is not confirmed, the Theil $U$ ratio does indicate that there are important numerical gains to using the TVT-NKPC procedure. Moreover, while the TVT-NKPC procedure does not significantly outperform the AO benchmark at any of the six horizons, the numerical gains of using it are considerable, beating the AO forecast by almost $20 \%$ at the longer horizons of 12, 16 and 20 quarters.

Overall, the findings for the US present more similarities than differences with respect to those for the EA. We can conclude that our TVT-NKPC forecasting procedure outperforms the conventional random walk benchmark at all horizons, significantly at 1 and 8 quarters in the EA and US data, and also at the longer run of 16 and 20 quarters in the EA case. Moreover, it also outperforms quantitatively, by about 10 to 20 percentage points in general, beyond the short run of 1 and 4 quarters, the agnostic AO benchmark that previous studies have found difficult to beat, significantly at the medium run of 8 and 12 quarters in the US case.

\footnotetext{
${ }^{23}$ Figures 15 (short run), 17 (medium run) and 19 (long run) in Appendix C provide a visual illustration for the best-performing variant of our TVT-NKPC forecasts against actual inflation in the US.
} 
While our task was not to study DSGE-based forecasts of inflation, and compare our method against these, we, finally here, also relate - briefly and indirectly - the forecasting performance of the TVT-NKPC procedure to that of a key representative of what may be termed structural models estimated using full information methods. Our choice of such a benchmark is taken from Faust and Wright (2013), who study - among their many other forecasting models we mentioned earlier - the predictive power of a DSGE model with time-varying inflation trend (labeled by them as the "DSGE-GAP" model) in US quarterly data. Using the respective numbers reported in their paper to calculate the corresponding Theil $\mathrm{U}$ ratios relative to the $\mathrm{AO}$ forecast, as in our tables 2 and $3,{ }^{24}$ we find that the DSGE-GAP model, which also incorporates "theory consistent" reduced-form parameters, does not perform significantly or distinguishably better than our TVT-NKPC forecasts. More precisely, the Theil U ratios of the Faust-Wright (2013) DSGE-GAP model to the AO model at horizons of 1, 4 and 8 quarters are, respectively 1.06, 1.01 and 1.00, which is similar to the accuracy reflected in our tables 2 and 3 for the TVT-NKPC procedure. Observe as well that, as already stressed, the predictive power of our procedure becomes stronger than that of the AO forecast especially at the longer end of the prediction horizons, namely at 12, 16 and 20 quarters, which range beyond what Faust and Wright (2013) have documented for their DSGE-GAP model. All in all, we see that the TVT-NKPC procedure does not predict less precisely than the DSGE-GAP model of Faust and Wright (2013) at the reported shorter horizons, and may even have an advantage at longer horizons - which remains to be explored in future work.

\section{Concluding Comments}

Previous results reported in the inflation forecasting literature have suggested some skepticism concerning the value added of theory-based models relative to the RW and AO forecasting benchmarks. Yet these models have typically exploited variants of the traditional and rather simple Phillips curve or of the New Keynesian Phillips Curve derived around a zero or constant trend inflation steady state in either single-equation estimation or DSGE system estimation, with King and Watson (2012) comparing the latter two alternative NKPC-based approaches and focusing on the choice of alternative real unit labor cost measures and some ensuing contradictory findings. This study has instead proposed a generalized New Keynesian Phillips Curve derived around a time-varying inflation trend with an additional proxy for unobservable marginal costs constructed from four observable time series arising from a monetary open-economy model that proved useful in the shortest and longest end of the forecast horizon we examined. On this basis, the corresponding model concept of fundamental inflation employed to predict the inflation

\footnotetext{
${ }^{24}$ Note, however, that the sample and forecasting evaluation periods considered by Faust and Wright (2013) are somewhat different from ours, 1960:1-2011:4 and 1985:1-2011:4, respectively.
} 
cycle captured in the NKPC has been combined with a theory-informed but also datasupported prediction for the time-varying trend (TVT) to obtain a TVT-NKPC forecast for actual inflation. In effect, our limited information approach to forecasting inflation offers a simpler alternative to the recent advances in better predicting of inflation that is based on full-information DSGE systems (see, e.g., Cai et al., 2018). We believe that both these theory/NKPC approaches have their strengths and weaknesses and should be used as complementary to each other.

Our results on comparative forecasting accuracy, using two quarterly data sets, namely, for the EA and the US over 1970:1-2015:4, that include some quite variable inflation periods, suggest that there is a role for theory: in both data sets the TVT-NKPC forecasts were overall much more accurate numerically than the AO benchmark (by 10-20 percentage points in the medium to longer run), and sometimes in a statistically significant way too, as in the US data at the medium-run horizons of 8 and 12 quarters ahead, that has been found to be quite difficult to beat in previous comparative studies (e.g., Atkeson and Ohanian, 2001; Stock and Watson, 2007, 2009; Faust and Wright, 2013). These results go some way to re-establishing confidence in the merit of Koopmans's dictum that we should 'resort to theory' to guide policy - and, we would add, empirical work and forecasting.

This study is part of continuing research seeking to build better theory-based forecasting procedures, which in practice combine with econometric methods of implementation. Present lines of enquiry include searching for an optimal length of the re-estimation window, considering alternative methods of trend adjustment, and robustifying the forecasts to possible structural breaks (see, for example, Castle et al., 2016), all of which may improve the TVT-NKPC forecasts, and including metrics other than quadratic loss to evaluate the forecasts.

\section{References}

[1] Adolfson, M., Lindé, J., Villani, M., 2007. Forecasting performance of an open economy DSGE model for the euro area. Econometric Reviews 26, 289-328.

[2] Andrews, D.W.K., 1991. Heteroskedasticity and autocorrelation consistent covariance matrix estimation. Econometrica 59, 817-858.

[3] Atkeson, A., Ohanian, L.E., 2001. Are Phillips curves useful for forecasting inflation? Quarterly Review, Federal Reserve Bank of Minneapolis 25, 2-11.

[4] Ball, L., Mazumder, S., 2011. Inflation dynamics and the great recession. Brookings Papers on Economic Activity 42, 337-405.

[5] Bartlett, M.S., 1950. Tests of significance in factor analysis. British Journal of Mathematical and Statistical Psychology 3, 77-85. 
[6] Bauwens, L., Koop, G., Korobilis, D., Rombouts, J.V.K., 2015. The contribution of structural break models to forecasting macroeconomic series. Journal of Applied Econometrics 30, 596-620.

[7] Cai, M., Del Negro, M., Giannoni, M.P., Gupta, A., Li, P., Moszkowski, E., 2018. DSGE forecasts of the lost recovery. Staff Reports 844, Federal Reserve Bank of New York.

[8] Calvo, G., 1983. Staggered prices in a utility maximizing framework. Journal of Monetary Economics 12, 383-398.

[9] Castle, J.L., Clements, M.P., Hendry, D.F., 2016. An overview of forecasting facing breaks. Journal of Business Cycle Research 12, 3-23.

[10] Clark, T.E., Doh, T., 2014. Evaluating alternative models of trend inflation. International Journal of Forecasting 30, 426-448.

[11] Clark, T.E., McCracken. M.W., 2013. Advances in forecast evaluation. In: Elliott, G., Timmermann, A. (Eds.), Handbook of Economic Forecasting, Vol. 2A. Elsevier.

[12] Cogley, T., Sbordone, A., 2008. Trend inflation, indexation, and inflation persistence in the new Keynesian Phillips curve. American Economic Review 98, 2101-2126.

[13] Dickey, D.A., Fuller, W.A., 1979. Distribution of the estimators for autoregressive time series with a unit root. Journal of the American Statistical Association 74, 427-431.

[14] Diebold, F.X., Mariano, R.S., 1995. Comparing predictive accuracy. Journal of Business and Economic Statistics 13, 253-263.

[15] Dotsey, M., Fujita, S., Stark, T., 2018. Do Phillips curves conditionally help to forecast inflation? International Journal of Central Banking 14, 43-92.

[16] Engle, R.F., Granger, C.W.J., 1987. Co-integration and error correction: Representation, estimation, and testing. Econometrica 55, 251-276.

[17] Edge, R., Gürkaynak, R.S., 2010. How useful are estimated DSGE model forecasts for central bankers? Brookings Papers on Economic Activity 41, 209-244.

[18] Faust, J., Wright, J., 2013. Forecasting inflation. In: Elliott, G., Timmermann, A. (Eds.), Handbook of Economic Forecasting, Vol. 2A. Elsevier.

[19] Galí, J., Gertler, M., 1999. Inflation dynamics: A structural econometric approach. Journal of Monetary Economics 44, 195-222. 
[20] Galí, J., Gertler, M., López-Salido, D., 2001. European inflation dynamics. European Economic Review 45, 1237-1270.

[21] Galí, J., Gertler, M., López-Salido, D., 2003. Erratum to European inflation dynamics. European Economic Review 47, 759-760.

[22] Galí, J., Gertler, M., López-Salido, D., 2005. Robustness of estimates of the hybrid new Keynesian Phillips curve. Journal of Monetary Economics 52, 1107-1118.

[23] Golyandina, N., Nekrutkin, V., Zhigljavsky, A.A., 2001. Analysis of Time Series Structure: SSA and Related Techniques. Chapman and Hall / CRS: Boca Raton.

[24] Hansen, L.P., 1982. Large sample properties of generalized method of moments estimators. Econometrica 50, 1029-1054.

[25] Harvey, A., 1989. Forecasting, Structural Time Series Models and the Kalman Filter. Cambridge: Cambridge University Press.

[26] Harvey, D., Leybourne, S., Newbold, P., 1997. Testing the equality of prediction mean squared errors. International Journal of Forecasting 13, 281-291.

[27] Hassani, H., Heravi, S., Zhigljavsky, A.A., 2009. Forecasting European industrial production with singular spectrum analysis. International Journal of Forecasting 25, $103-118$.

[28] Hodrick, R.J., Prescott, E.C., 1997. Postwar US business cycles: An empirical investigation. Journal of Money, Credit and Banking 29, 1-16.

[29] Johansen, S., 1996. Likelihood-Based Inference in Cointegrated Vector AutoRegressive Models. Oxford: Oxford University Press.

[30] Kascha, C., Ravazzolo, F., 2010. Combining inflation density forecasts. Journal of Forecasting 29, 231-250.

[31] Kichian, M., Rumler, F., 2014. Forecasting Canadian inflation: A semi-structural NKPC approach. Economic Modelling 43, 183-191.

[32] King, R.G., Watson, M.W., 2012. Inflation and unit labor costs. Journal of Money, Credit and Banking 44, 111-149.

[33] Koopmans, T., 1947. Measurement without theory. Review of Economics and Statistics $29,161-172$.

[34] Kwiatkowski, D., Phillips, P.C.B., Schmidt, P., Shin, Y., 1992. Testing the null hypothesis of stationarity against the alternative of a unit root. Journal of Econometrics 54, 159-178. 
[35] Leith, C., Malley, J., 2007. Estimated open economy new Keynesian Phillips curves for the G7. Open Economies Review 18, 405-426.

[36] Liu, D., Jansen, D.W., 2011. Does a factor Phillips curve help? An evaluation of the predictive power for US inflation. Empirical Economics 40, 807-826.

[37] Lütkepohl, H., 1987. Forecasting Aggregated Vector ARMA Processes. SpringerVerlag.

[38] MacKinnon, J., 1996. Numerical distribution functions for unit root and cointegration tests. Journal of Applied Econometrics 11, 601-618.

[39] McKnight, S., Mihailov, A., 2015. Do real balance effects invalidate the Taylor principle in closed and open economies? Economica 82, 938-975.

[40] Meese, R.A., Rogoff, K., 1983. Empirical exchange rate models of the seventies? Do they fit out of sample? Journal of International Economics 14, 3-24.

[41] Mihailov, A., Rumler, F., Scharler, J., 2011a. The small open-economy new Keynesian Phillips curve: Empirical evidence and implied inflation dynamics. Open Economies Review 22, 317-337.

[42] Mihailov, A., Rumler, F., Scharler, J., 2011b. Inflation dynamics in the new EU member states: How relevant are external factors? Review of International Economics $19,65-76$.

[43] Newey, W.K., West, K.D., 1987. A simple, positive semi-definite, heteroskedasticity and autocorrelation consistent covariance matrix. Econometrica 55, 703-708.

[44] Patterson, K.D., Hassani, H., Heravi, S., Zhigljavsky, A.A., 2010. Multivariate singular spectrum analysis for forecasting revisions to real-time data. Journal of Applied Statistics 38, 2183-2211.

[45] Posch, J., Rumler, F., 2015. Semi-structural forecasting of UK inflation based on the hybrid new Keynesian Phillips curve. Journal of Forecasting 34, 145-162.

[46] Rossi, B., Sekhposyan, T., 2010. Have economic models' forecasting performance for US output growth and inflation changed over time, and when? International Journal of Forecasting 26, 800-835.

[47] Rossi, B., Sekhposyan, T., 2014. Evaluating predictive densities of US output growth and inflation in a large macroeconomic data set. International Journal of Forecasting $30,662-682$. 
[48] Rumler, F., 2007. Estimates of the open economy new Keynesian Phillips curve for euro area countries. Open Economies Review 18, 427-451.

[49] Rumler, F., Valderrama, M.T., 2010. Comparing the new Keynesian Phillips curve with time series models to forecast inflation. North American Journal of Economics and Finance 21, 126-144.

[50] Said, S.E., Dickey, D.A., 1984. Testing for unit roots in autoregressive movingaverage models with unknown order. Biometrika 71, 599-607.

[51] Sbordone, A., 2002. Prices and unit labor costs: A new test of price stickiness. Journal of Monetary Economics 49, 235-456.

[52] Sbordone, A., 2005. Do expected future marginal costs drive inflation dynamics? Journal of Monetary Economics 52, 1183-1197.

[53] Schwarz, G.E., 1978. Estimating the dimension of a model. Annals of Statistics 6, 461-464.

[54] Stock, J.H., Watson, M.W., 1999. Forecasting inflation. Journal of Monetary Economics 44, 293-335.

[55] Stock, J.H., Watson, M.W., 2003. Forecasting output and inflation: The role of asset prices. Journal of Economic Literature 41, 788-829.

[56] Stock, J.H., Watson, M.W., 2007. Why has US inflation become harder to forecast? Journal of Money, Credit and Banking 39, 3-34.

[57] Stock, J.H., Watson, M.W., 2009. Phillips curve inflation forecasts. In: Fuhrer, J., Kodrzycki, Y., Little, J., Olivei, J. (Eds.), Understanding Inflation and the Implications for Monetary Policy. Cambridge, MA: MIT Press.

[58] Woodford, M., 2003. Interest Rates and Prices: Foundations of a Theory of Monetary Policy. Princeton, NJ: Princeton University Press.

[59] Yazgan, M.E., Yilmazkuday, H., 2007. Monetary policy rules in practice: Evidence from Turkey and Israel. Applied Financial Economics 17, 1-8.

[60] Yilmazkuday, H., 2007. Inflation targeting supported by managed exchange rate. Applied Economics 39, 2011-2026. 


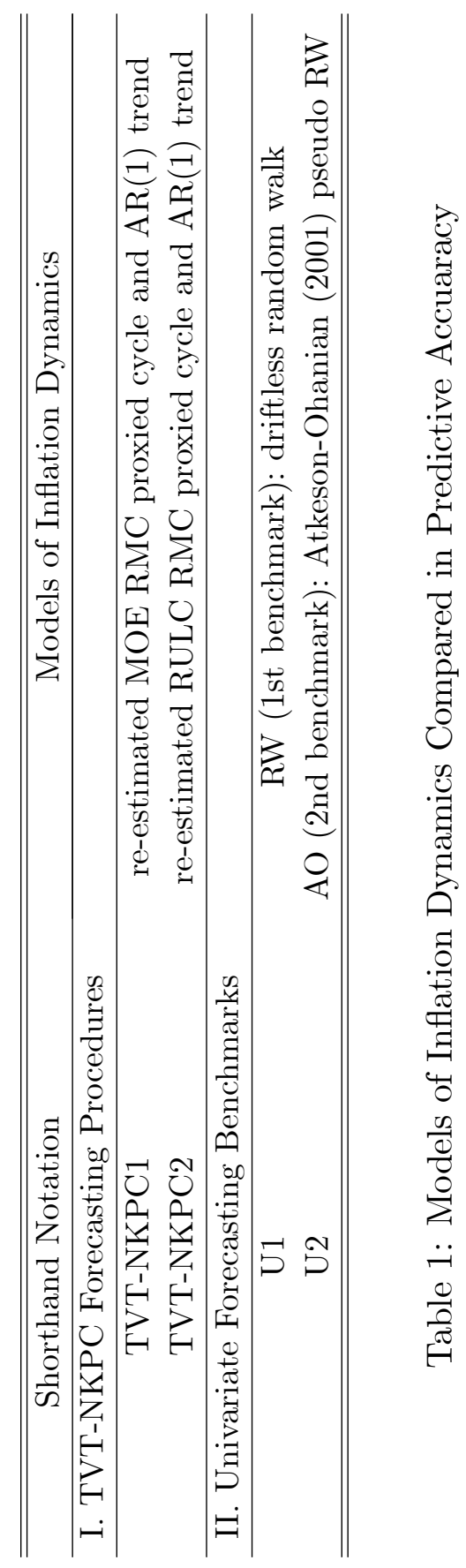

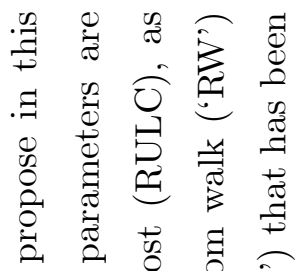

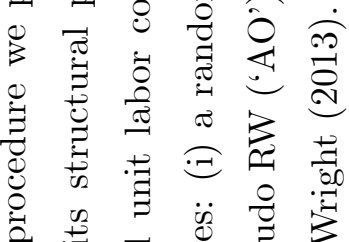

a

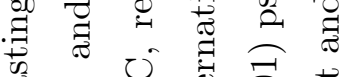

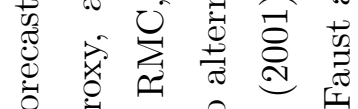

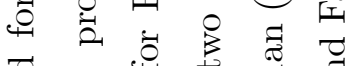

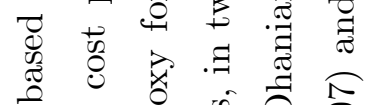

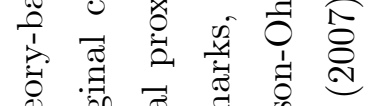

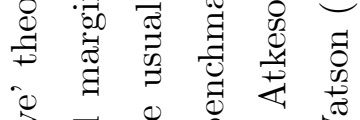

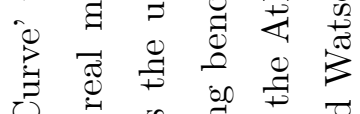

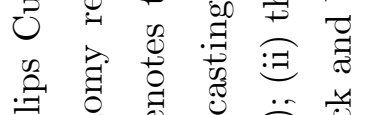

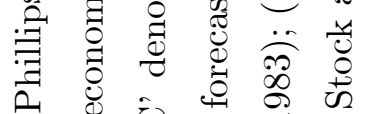

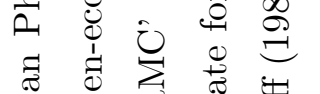

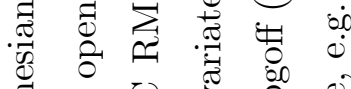

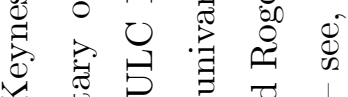

$\checkmark$ 艺

范

च

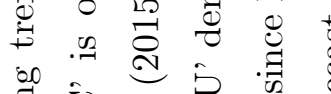

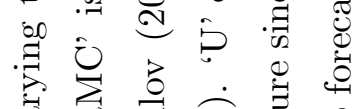

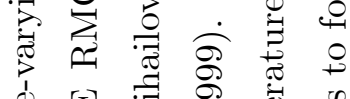

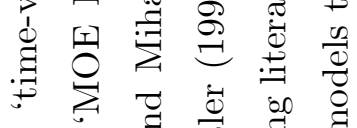

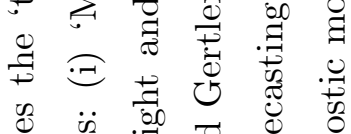

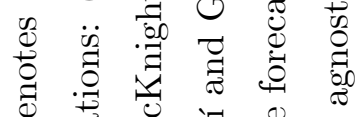

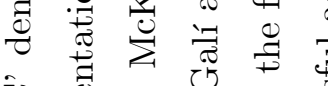

ن

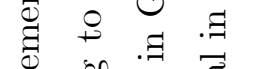

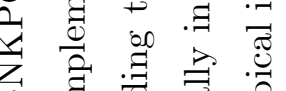

.

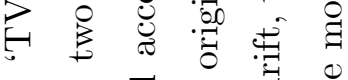

ن.

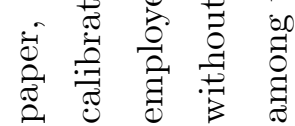




\begin{tabular}{|c|c|c|c|c|c|c|}
\hline \multirow{2}{*}{$\begin{array}{c}\text { Forecasting evaluation period } \\
\text { Forecast horizon, quarters }\end{array}$} & \multicolumn{6}{|c|}{ 2000:1-2015:4 } \\
\hline & 1 & 4 & 8 & 12 & 16 & 20 \\
\hline \multicolumn{7}{|c|}{ Panel A: Root MSFE } \\
\hline \multicolumn{7}{|c|}{ Theory-Based TVT-NKPC Procedures of Inflation Forecasting } \\
\hline MOE RMC (roll) & 0.729 & 0.884 & 0.912 & 0.829 & 0.745 & 0.883 \\
\hline MOE RMC (rec) & 0.706 & 0.879 & 0.914 & 0.842 & 0.759 & 0.838 \\
\hline RULC RMC (roll) & 0.751 & 0.873 & 0.892 & 0.816 & 0.739 & 0.880 \\
\hline RULC RMC (rec) & 0.765 & 0.879 & 0.892 & 0.822 & 0.747 & 0.833 \\
\hline \multicolumn{7}{|c|}{ Agnostic Univariate Benchmarks of Inflation Forecasting } \\
\hline RW Forecast & 0.909 & 0.885 & 1.038 & 1.001 & 0.996 & 1.092 \\
\hline AO (Pseudo-RW) Forecast & 0.669 & 0.815 & 0.913 & 0.894 & 0.819 & 0.921 \\
\hline \multicolumn{7}{|c|}{ Panel B: Theil U-stat to RW $\equiv$ root MSFE of TVT-NKPC w.r.t. RW forecast } \\
\hline MOE RMC (roll) & $0.802^{* * *}$ & 1.000 & 0.879 & 0.821 & $0.748^{*}$ & 0.808 \\
\hline MOE RMC (rec) & $0.776^{* * *}$ & 0.993 & 0.881 & 0.834 & $0.762^{*}$ & $0.767^{*}$ \\
\hline RULC RMC (roll) & $0.826^{* *}$ & 0.987 & $0.859^{*}$ & 0.809 & $0.742^{*}$ & 0.805 \\
\hline RULC RMC (rec) & $0.842^{* *}$ & 0.993 & $0.860^{*}$ & 0.814 & $0.751^{*}$ & $0.763^{*}$ \\
\hline \multicolumn{7}{|c|}{ Panel C: Theil U-stat to AO $\equiv$ root MSFE of TVT-NKPC w.r.t. AO forecast } \\
\hline MOE RMC (roll) & $1.090^{* *}$ & 1.086 & 0.999 & 0.927 & 0.909 & 0.958 \\
\hline MOE RMC (rec) & 1.053 & 1.078 & 1.001 & 0.942 & 0.926 & 0.910 \\
\hline RULC RMC (roll) & $1.121^{* * *}$ & 1.072 & 0.977 & 0.914 & 0.901 & 0.955 \\
\hline RULC RMC (rec) & $1.143^{* *}$ & $1.079^{*}$ & 0.977 & 0.920 & 0.912 & 0.905 \\
\hline
\end{tabular}

Table 2: Predictive Performance of TVT-NKPC Forecasts in the EA Data - MDM Test

Note: Bold font indicates the best forecast by horizon. All forecasts are iterated (see online Appendix B.3), and implemented in two versions; (i) 'roll' denotes fixed-length rolling window; (ii) 'rec' denotes augmenting-length recursive window. 'TVT-NKPC' is eq. (4), derived in online Appendices B.1 and B.2; its reduced-form parameters $\gamma$ and $\kappa$-after calibrating $\rho=0.2$, see Adolfson et al. (2007) and Cogley and Sborodone (2008) - are re-estimated in the pseudo-outof-sample forecasting simulation via GMM using 5 lags of the dependent variable and 6 lags of the other variables in eq. (4) as instruments. As discussed in section 2, we employ two proxies for real marginal cost (RMC) in eq. (4): (i) 'MOE RMC' denotes a monetary openeconomy RMC proxy; (ii) 'RULC RMC' denotes a real unit labor cost proxy. The modified Diebold-Mariano (1995) t-statistic (see Harvey et al., 1997) with p-values using Newey-West (1987) HAC standard errors, tests the null of no significant difference in the forecast accuracy of two compared (non-nested) models; statistical significance of the (one-sided) test is shown at conventional levels: $* * * 1 \%, * * 5 \%$, and $* 10 \%$. These results are robust to applying instead the original Diebold-Mariano (one-sided) test - see Table 2 in Appendix C (online). 


\begin{tabular}{|c|c|c|c|c|c|c|}
\hline \multirow{2}{*}{$\begin{array}{c}\text { Forecasting evaluation period } \\
\text { Forecast horizon, quarters }\end{array}$} & \multicolumn{6}{|c|}{ 2000:1-2015:4 } \\
\hline & 1 & 4 & 8 & 12 & 16 & 20 \\
\hline \multicolumn{7}{|c|}{ Panel A: Root MSFE } \\
\hline \multicolumn{7}{|c|}{ Theory-Based TVT-NKPC Procedures of Inflation Forecasting } \\
\hline MOE RMC (roll) & 0.854 & 1.156 & 1.165 & 1.249 & 1.255 & 1.662 \\
\hline MOE RMC (rec) & 0.986 & 1.125 & 1.438 & 1.370 & 1.291 & 1.144 \\
\hline RULC RMC (roll) & 0.914 & 1.041 & 1.100 & 1.229 & 1.286 & 1.655 \\
\hline RULC RMC (rec) & 0.922 & 1.036 & 1.127 & 1.227 & 1.261 & 1.179 \\
\hline \multicolumn{7}{|c|}{ Agnostic Univariate Benchmarks of Inflation Forecasting } \\
\hline RW Forecast & 0.970 & 1.127 & 1.333 & 1.360 & 1.470 & 1.447 \\
\hline AO (Pseudo-RW) Forecast & 0.868 & 1.027 & 1.222 & 1.344 & 1.376 & 1.251 \\
\hline \multicolumn{7}{|c|}{ Panel B: Theil U-stat to RW $\equiv$ root MSFE of TVT-NKPC w.r.t. RW forecast } \\
\hline MOE RMC (roll) & $0.881^{*}$ & 1.034 & 0.874 & 0.918 & 0.854 & 1.149 \\
\hline MOE RMC (rec) & 1.016 & 0.998 & 1.079 & 1.007 & 0.878 & 0.790 \\
\hline RULC RMC (roll) & 0.942 & 0.924 & $0.826^{*}$ & 0.903 & 0.875 & 1.114 \\
\hline RULC RMC (rec) & 0.950 & 0.919 & $0.845^{*}$ & 0.902 & 0.858 & 0.812 \\
\hline \multicolumn{7}{|c|}{ Panel C: Theil U-stat to AO $\equiv$ root MSFE of TVT-NKPC w.r.t. AO forecast } \\
\hline MOE RMC (roll) & 0.984 & $1.134^{* *}$ & 0.953 & 0.929 & 0.912 & 1.329 \\
\hline MOE RMC (rec) & $1.135^{* * *}$ & 1.096 & $1.177^{*}$ & 1.020 & 0.938 & 0.914 \\
\hline RULC RMC (roll) & 1.053 & 1.014 & $0.901^{* *}$ & $0.914^{*}$ & 0.935 & 1.323 \\
\hline RULC RMC (rec) & 1.061 & 1.001 & $0.922^{* *}$ & $0.913^{*}$ & 0.916 & 0.943 \\
\hline
\end{tabular}

Table 3: Predictive Performance of TVT-NKPC Forecasts in the US Data - MDM Test

Note: See the note below Table 2 and Table 3 in Appendix C (online). 


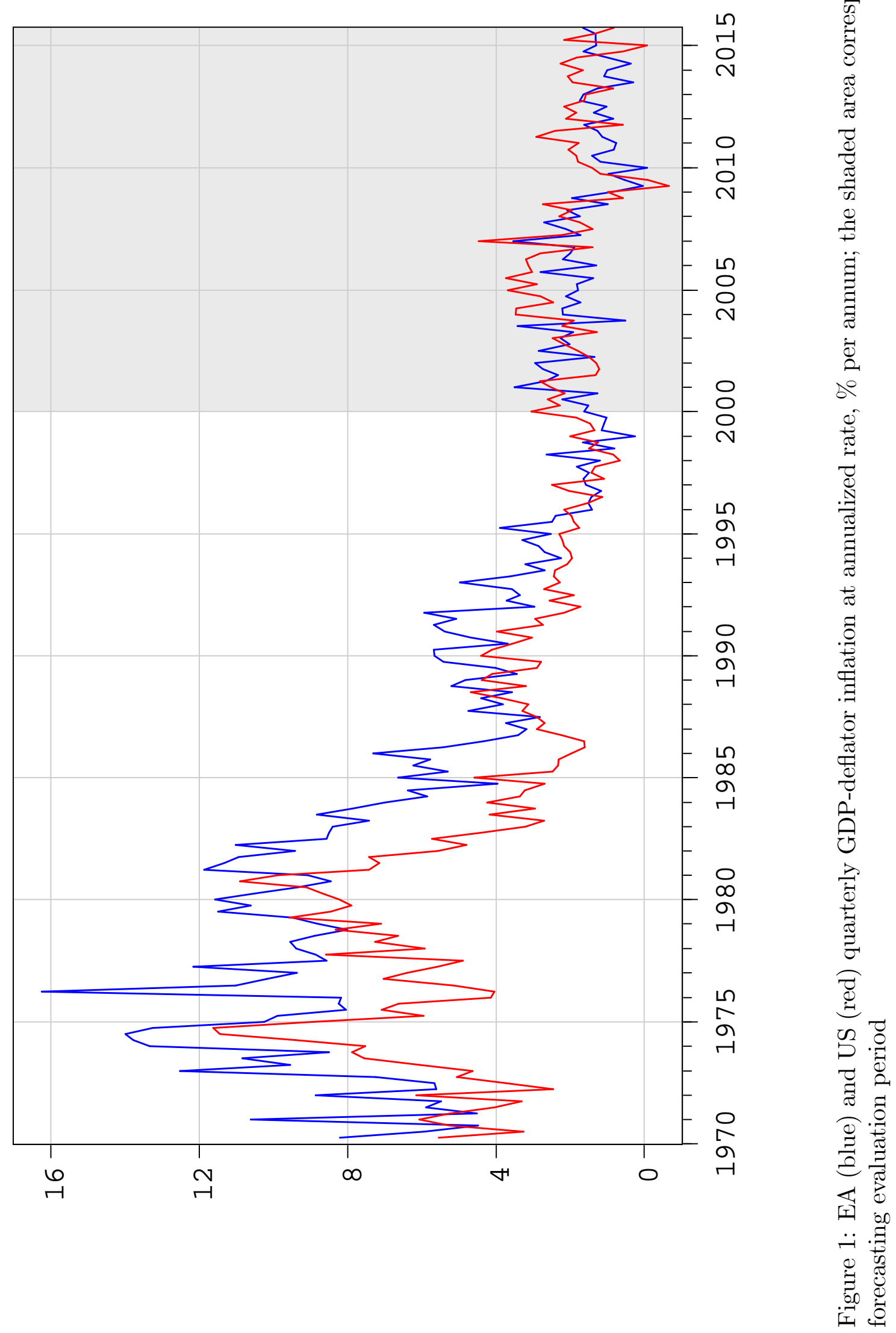




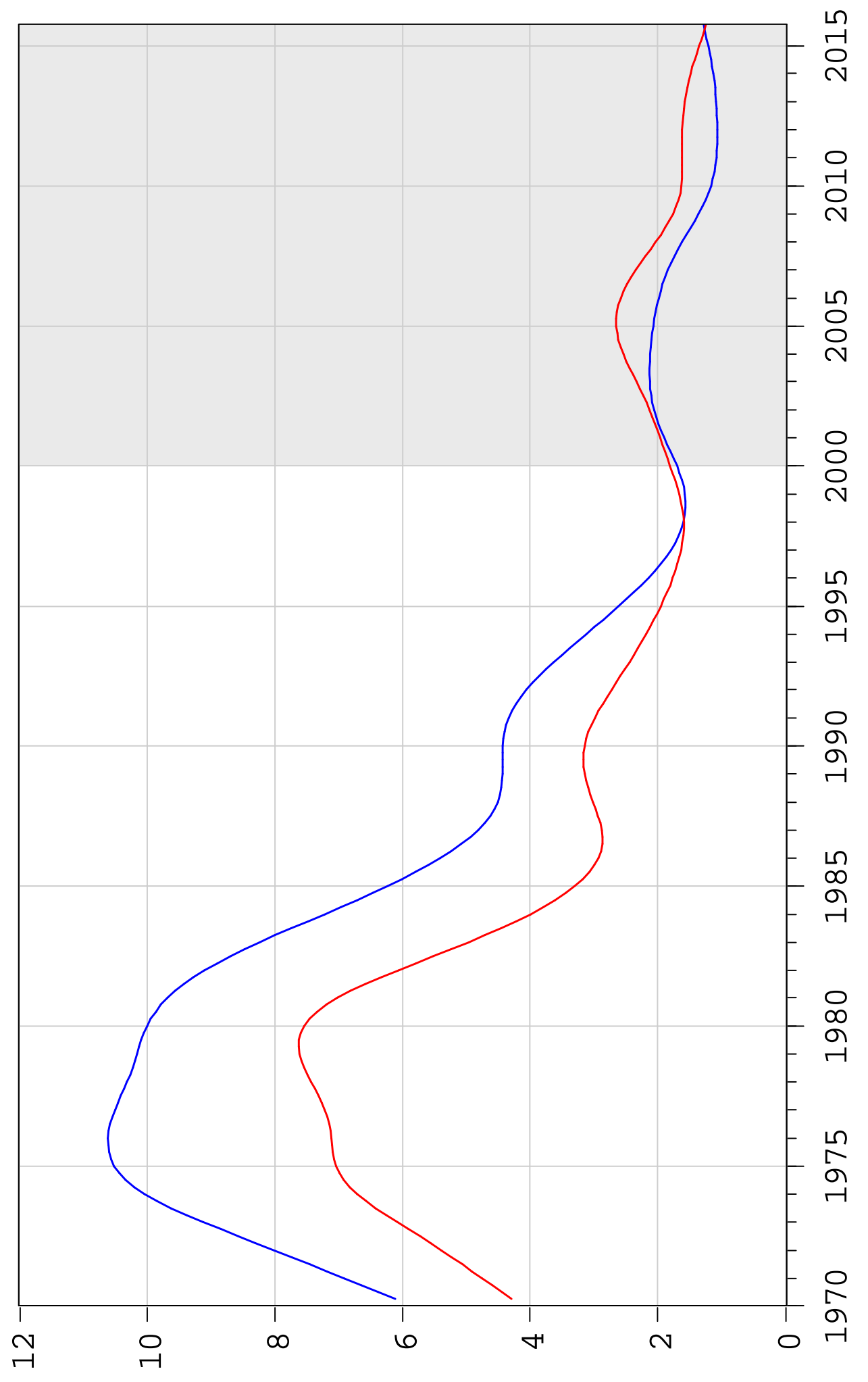




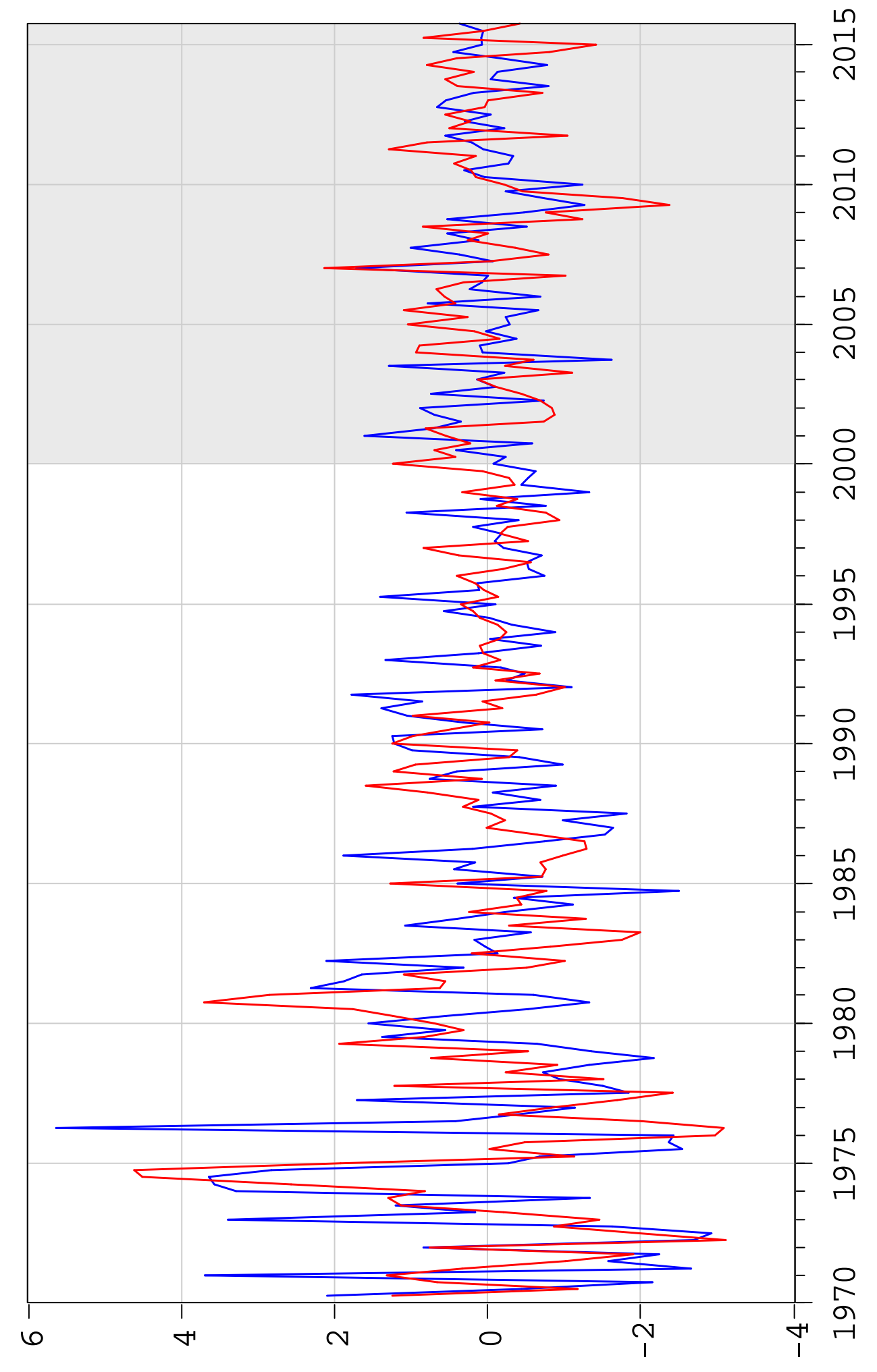




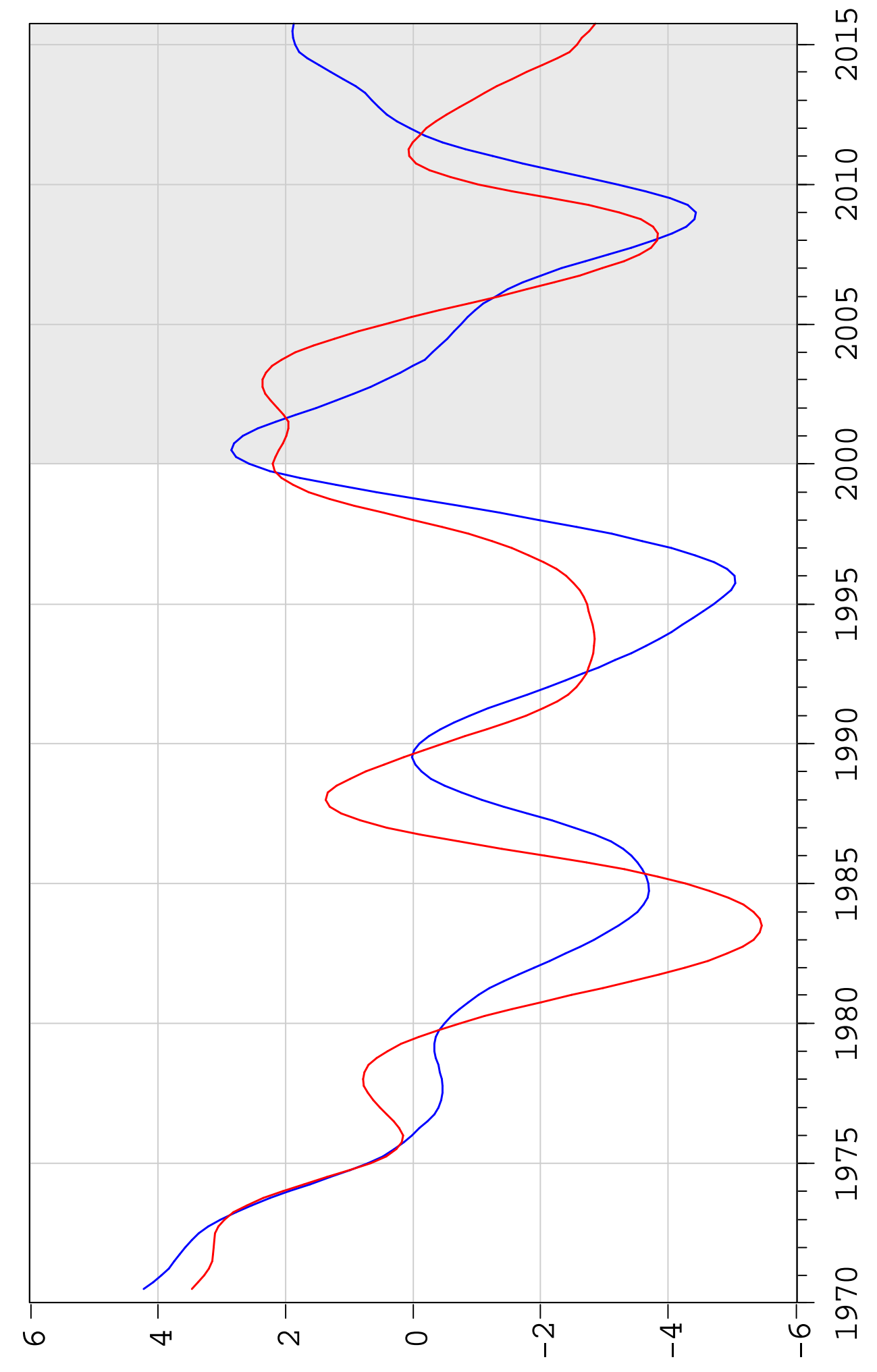





\title{
For online publication: Supplementary Appendix to "Inflation Forecasting Using the New Keynesian Phillips Curve with a Time-Varying Trend"
}

\author{
Stephen McKnight* Alexander Mihailov ${ }^{\dagger}$ Fabio Rumler ${ }^{\ddagger}$ \\ Accepted (pre-print) version at Economic Modelling on 14 August 2019
}

\begin{abstract}
This supplementary online appendix provides the sources and definitions of our data (in section A) as well as further technical derivations and details (in section B). It also presents and briefly discusses additional results on stationarity tests and robustness, in appendix tables, and illustrates our forecasting procedure, in appendix figures (in section C): some of these additional tests, results and illustrations are also referred to in the main text. For replication purposes, a zip file archive is available on the journal website that contains our data set, codes and the respective input and output files (using the econometric software package EViews, version 9.5 - but also works with the earlier versions 9.0, 8.1 and 7.2; the one-sided Hodrick-Prescott filter add-in needs to be downloaded from the EViews website).
\end{abstract}

\footnotetext{
*Centro de Estudios Económicos, El Colegio de México, Camino al Ajusco 20, Col. Pedregal de Sta. Teresa, México D.F., C.P. 10740, México; mcknight@colmex.mx

${ }^{\dagger}$ Corresponding author: Department of Economics, University of Reading, Whiteknights, Reading RG6 6AA, United Kingdom; a.mihailov@reading.ac.uk

${ }^{\ddagger}$ Oesterreichische Nationalbank, Economic Analysis Division, Otto Wagner Platz 3, A-1090 Vienna, Austria; fabio.rumler@oenb.at
} 


\section{Contents}

A Data Sources and Definitions 1

A.1 Euro Area . . . . . . . . . . . . . . . . . . . . . . . . 1

A.2 United States . . . . . . . . . . . . . . . . . . . . . 2

B Technical Appendix 3

B.1 Derivation of a Generalized NKPC with Drifting Trend Inflation . . . . . . . . 3

B.1.1 Log-Linear Optimality Condition of Price-Setting Firms . . . . . . . . . 3

B.1.2 Log-Linear Aggregate Price Level and the TVT-NKPC . . . . . . . . 8

B.2 Log-Linear Marginal Cost Condition of McKnight and Mihailov (2015) . . . . . 9

B.3 Alternative Types of Multistep Univariate Forecasts . . . . . . . . . . . . . . 12

B.3.1 Iterated Multistep Univariate Forecasts . . . . . . . . . . . . . . . . . 12

B.3.2 Direct Multistep Univariate Forecasts . . . . . . . . . . . . . . . . . 13

C Additional Results $\quad 14$

C.1 Stationarity Tests and Further Robustness Checks . . . . . . . . . . . . . . . . 14

C.2 Further Graphical Illustrations _ . . . . . . . . . . . . . . . . 18 


\section{List of Tables}

1 Stationarity Tests for the Components and Drivers of the TVT-NKPC . . . . . 15

2 Predictive Performance of TVT-NKPC Forecasts in the EA Data - DM Test . . . 16

3 Predictive Performance of TVT-NKPC Forecasts in the US Data - DM Test . . . 17

\section{List of Figures}

1 US - Our Trend Inflation Measure versus Common Alternatives . . . . . . . . . . 20

2 EA - GDP-Deflator Inflation versus HICP Inflation . . . . . . . . . . . . 21

3 US - GDP-Deflator Inflation versus CPI Inflation . . . . . . . . . . . . . . . . 22

4 EA and US - Cyclical Component of the MOE Measure of Real Marginal Cost . 23

$5 \quad$ EA and US - Cyclical Component of the RULC Measure of Real Marginal Cost . 24

$6 \quad$ EA - Recursively Re-estimated Persistence of Trend Inflation . . . . . . . . . . 25

$7 \quad$ US - Recursively Re-estimated Persistence of Trend Inflation . . . . . . . . . . . 26

$8 \quad$ EA - Recursively Re-estimated Persistence of Trend Inflation Growth . . . . . . 27

$9 \quad$ US - Recursively Re-estimated Persistence of Trend Inflation Growth . . . . . . 28

10 EA - Recursively Re-estimated TVT-NKPC Slope (MOE RMC Proxy) . . . . . 29

11 US - Recursively Re-estimated TVT-NKPC Slope (MOE RMC Proxy) . . . . . . 30

12 EA - Recursively Re-estimated TVT-NKPC Slope (RULC Proxy) . . . . . . . . 31

13 US - Recursively Re-estimated TVT-NKPC Slope (RULC Proxy) . . . . . . . . 32

14 EA - Best TVT-NKPC Short-Run (1- and 4-Quarters Ahead) Forecasts . . . . . 33

15 US - Best TVT-NKPC Short-Run (1- and 4-Quarters Ahead) Forecasts . . . . . 34

16 EA - Best TVT-NKPC Medium-Run (8- and 12-Quarters Ahead) Forecasts . . . 35

17 US - Best TVT-NKPC Medium-Run (8- and 12-Quarters Ahead) Forecasts . . . 36

18 EA - Best TVT-NKPC Long-Run (16- and 20-Quarters Ahead) Forecasts . . . . 37

19 US - Best TVT-NKPC Long-Run (16- and 20-Quarters Ahead) Forecasts . . . . 38 



\section{A Data Sources and Definitions}

This Appendix A provides details on our data sources and definitions.

\section{A.1 Euro Area}

- Source: 16th update to the database underlying the Area Wide Model (AWM), see Fagan, Henry and Mestre (2005) and their ECB (2001) working paper at http://www.ecb.europa.eu/; the units of the series follow Eurostat or ECB conventions, that is:

- real GDP and its components are in millions of ECU/euro corrected with reference year 1995;

- nominal series are (typically) in millions of ECU/euro corrected;

- deflators are (generally) set to 1 in 1995.

- Mnemonics and Definitions - all variables are released at quarterly frequency and as seasonally adjusted at the data source:

- EAMTD $\Leftrightarrow$ MTD in the AWM: Imports of Goods and Services Deflator;

- EAPCR $\Leftrightarrow \mathrm{PCR}$ in the AWM: Private Consumption (Real);

$-\mathrm{EATOT} \equiv \mathrm{EAMTD} / \mathrm{EAXTD}$

- EARULC $\Leftrightarrow$ ULC in the AWM: Unit Labour Costs, calculated as the ratio of compensation of employees to real GDP (ULC = WIN / YER);

- EAXTD $\Leftrightarrow$ XTD in the AWM: Exports of Goods and Services Deflator;

- EAYED $\Leftrightarrow$ YED in the AWM: GDP Deflator;

- EAYER $\Leftrightarrow$ YER in the AWM: GDP (Real).

- Source: FRED (Federal Reserve Economic Data), Economic Research Division, Federal Reserve Bank of St. Louis; http://research.stlouisfed.org/fred2/ (accessed on 2 September 2017)

- Mnemonics and Definitions:

- EAM1 $\Leftrightarrow$ MYAGM1EZQ196N at FRED via the International Monetary Fund / International Financial Statistics (IMF/IFS): M1 for Euro Area, Euros; Frequency: Quarterly, Not Seasonally Adjusted (subsequently seasonally adjusted, for consistency with the remaining raw data employed in the present study, using the Census$\mathrm{X} 12$ procedure);

$-\mathrm{EAM} 1 \mathrm{R} \equiv \mathrm{EAM} 1$ / EAYED. 


\section{A.2 United States}

- Source: FRED (Federal Reserve Economic Data), Economic Research Division, Federal Reserve Bank of St. Louis; http://research.stlouisfed.org/fred2/ (accessed on 2 September 2017)

- Mnemonics and Definitions - all variables are released at quarterly frequency and as seasonally adjusted at the respective original data source:

- USGDPD $\Leftrightarrow$ GDPDEF at FRED via the Bureau of Economic Analysis (BEA): Gross Domestic Product - Implicit Price Deflator, Index 2009=100, Quarterly, Seasonally Adjusted;

- USM1 $\Leftrightarrow$ MANMM101USQ189S at FRED via the Organization for Economic Cooperation and Development / Main Economic Indicators (OECD/MEI): M1 for the United States, National Currency, Quarterly, Seasonally Adjusted;

- USM1R $\equiv$ USM1 / USGDPD;

- USMD $\Leftrightarrow$ A021RD3Q086SBEA at FRED via BEA: Imports of Goods and Services - Implicit Price Deflator, Index 2009=100, Quarterly, Seasonally Adjusted;

- USRGDP $\Leftrightarrow$ GDPC1 at FRED via BEA: Real Gross Domestic Product, Billions of Chained 2009 Dollars, Quarterly, Seasonally Adjusted Annual Rate;

- USRPCE $\Leftrightarrow$ PCECC96 at FRED via BEA: Real Personal Consumption Expenditures, Billions of Chained 2009 Dollars, Quarterly, Seasonally Adjusted Annual Rate;

- USULC: ULCNFB_20120606 at FRED via BEA: Nonfarm Business Sector, Unit Labor Cost, Index 2009=100, Quarterly, Seasonally Adjusted;

- USRULC $\equiv$ USULC / USGDPD;

- USTOT $\equiv$ USMD / USXD;

- USXD $\Leftrightarrow$ A020RD3Q086SBEA at FRED via BEA: Exports of Goods and Services - Implicit Price Deflator, Index 2009=100, Quarterly, Seasonally Adjusted. 


\section{B Technical Appendix}

This Appendix B provides further technical details.

\section{B.1 Derivation of a Generalized NKPC with Drifting Trend Inflation}

In the present section, we provide the steps in the derivation of our generalized TVT-NKPC, eqs. (2) and (4) in the main text.

\section{B.1.1 Log-Linear Optimality Condition of Price-Setting Firms}

Denoting the cumulative gross inflation rate between dates $t$ and $t+s$ as

$$
\Pi_{t, t+s} \equiv \frac{P_{t+s}}{P_{t}}
$$

it is convenient to define

$$
\Psi_{t, t+s} \equiv \prod_{s=0}^{s-1} \Pi_{t+s}^{\rho} \bar{\Pi}_{t+s+1}^{1-\rho},
$$

with the normalization $\Psi_{t, t}=1$, which generalizes the analogous definition in Cogley and Sbordone (2008). The demand for intermediate good $i$ at $t+s$ if producer $i$ last reoptimized at $t$ is thus

$$
Y_{t, t+s}(i)=\left[\frac{\Psi_{t, t+s} P_{t}^{*}(i)}{P_{t+s}}\right]^{-\epsilon} Y_{t+s}
$$

where $P_{t}^{*}(i)$ is the optimal price for $i$ chosen at $t$.

As is standard, let us define the stochastic discount factor (or pricing kernel) as

$$
M_{t, t+s} \equiv \beta \frac{\Lambda_{t+s}}{\Lambda_{t}}
$$

where $\beta$ is the deterministic discount factor and $\Lambda_{t}$ denotes the marginal utility of wealth of firm owners.

It follows that if a producer $i$ is drawn to reset her price at time $t$, she will select $P_{t}^{*}(i)$ so as to maximize expected discounted future profits, ${ }^{1}$

$$
\max _{P_{t}^{*}} E_{t} \sum_{s=0}^{\infty}(\alpha \beta)^{s} \frac{\Lambda_{t+s}}{\Lambda_{t}}\left[\frac{\Psi_{t, t+s} P_{t}^{*}}{P_{t+s}} Y_{t, t+s}-T C_{t+s}\left(Y_{t, t+s}\right)\right]
$$

where $E_{t}$ is the expectation operator conditional on information available at time $t, Y_{t, t+s}$ is firms' (expected) output at their optimally set price in $t$, subject to the sequence of demand constraints in (2), and the time-varying function $T C_{t+s, t}(\cdot)$ is the (expected) real total cost function.

Substituting the demand function (2) in the objective (3),

\footnotetext{
${ }^{1}$ All firms that are given the opportunity to reset their price in period $t$ behave in an identical manner. Hence, $P_{t}^{*}(i)=P_{t}^{*}$.
} 


$$
E_{t} \sum_{s=0}^{\infty}(\alpha \beta)^{s} \frac{\Lambda_{t+s}}{\Lambda_{t}}\left[\left(\frac{\Psi_{t, t+s} P_{t}^{*}}{P_{t+s}}\right)^{1-\epsilon} Y_{t+s}-T C_{t+s}\left(\frac{\Psi_{t, t+s} P_{t}^{*}}{P_{t+s}}\right)^{-\epsilon} Y_{t+s}\right] .
$$

The first-order necessary condition (FONC) on $P_{t}^{*}$ is

$$
E_{t} \sum_{s=0}^{\infty}(\alpha \beta)^{s} \frac{\Lambda_{t+s}}{\Lambda_{t}}\left[\frac{\Psi_{t, t+s}}{P_{t+s}}\left(\frac{\Psi_{t, t+s} P_{t}^{*}}{P_{t+s}}\right)^{-\epsilon} Y_{t+s}-\frac{\epsilon}{\epsilon-1} \frac{\Psi_{t, t+s}}{P_{t+s}}\left(\frac{\Psi_{t, t+s} P_{t}^{*}}{P_{t+s}}\right)^{-\epsilon-1} Y_{t+s} M C_{t, t+s}\right]=0,
$$

where $M C_{t, t+s}$ depends on terms that are specific to the firms resetting their price at $t$ and not changing it through $t+s$,

$$
M C_{t, t+s}=M C_{t+s}\left[\frac{\Psi_{t, t+s} P_{t}^{*}}{P_{t+s}}\right]^{-\epsilon} Y_{t+s}
$$

and $M C_{t+s}$ denotes the average (or aggregate) real marginal cost in the model economy. Using the fact that the optimal price $P_{t}^{*}$ is known at $t$ and multiplying (4) by $P_{t}^{*}$, we obtain

$$
E_{t} \sum_{s=0}^{\infty}(\alpha \beta)^{s} \frac{\Lambda_{t+s}}{\Lambda_{t}}\left(\frac{\Psi_{t, t+s} P_{t}^{*}}{P_{t+s}}\right)^{-\epsilon} Y_{t+s}\left[\frac{\Psi_{t, t+s} P_{t}^{*}}{P_{t+s}}-\frac{\epsilon}{\epsilon-1} M C_{t, t+s}\right]=0
$$

Denoting the optimal relative price as

$$
p_{t}^{*} \equiv \frac{P_{t}^{*}}{P_{t}}
$$

allows to further express the FONC of price-setting firms in $t$ more compactly,

$$
E_{t} \sum_{s=0}^{\infty}(\alpha \beta)^{s} \frac{\Lambda_{t+s}}{\Lambda_{t}}\left(\frac{\Psi_{t, t+s}}{\Pi_{t, t+s}} p_{t}^{*}\right)^{-\epsilon} Y_{t+s}\left[\frac{\Psi_{t, t+s}}{\Pi_{t, t+s}} p_{t}^{*}-\frac{\epsilon}{\epsilon-1} M C_{t, t+s}\right]=0 .
$$

With these assumptions on price-setting, the aggregate price level evolves according to

$$
P_{t}=\left[(1-\alpha)\left(P_{t}^{*}\right)^{1-\epsilon}+\alpha\left(\Pi_{t-1}^{\rho} \bar{\Pi}_{t}^{1-\rho} P_{t-1}\right)^{1-\epsilon}\right]^{\frac{1}{1-\epsilon}}
$$

which can also be written in terms of the optimal relative price as

$$
1=(1-\alpha)\left(p_{t}^{*}\right)^{1-\epsilon}+\alpha\left(\frac{\Pi_{t-1}^{\rho} \bar{\Pi}_{t}^{1-\rho}}{\Pi_{t}}\right)^{1-\epsilon} .
$$

In line with the New Keynesian literature, the individual real marginal cost at $t+s$ of a firm $i$ drawn to set optimally its price at $t$, denoted as $M C_{t, t+s}$, is assumed to be related to the average (or aggregate) real marginal cost in the model economy, $M C_{t+s}$, according to

$$
M C_{t, t+s}=M C_{t+s}\left[\left(\frac{\Psi_{t, t+s} P_{t}^{*}}{P_{t+s}}\right)^{-\epsilon}\right]^{\omega}=M C_{t+s}\left(\frac{\Psi_{t, t+s}}{\Pi_{t, t+s}} p_{t}^{*}\right)^{-\epsilon \omega}
$$


where $\omega$ denotes the elasticity of firm $i$ 's real marginal cost to its own real output. Substituting the above expression in the firm's price-setting FONC yields

$$
E_{t} \sum_{s=0}^{\infty}(\alpha \beta)^{s} \frac{\Lambda_{t+s}}{\Lambda_{t}}\left(\frac{\Psi_{t, t+s}}{\Pi_{t, t+s}} p_{t}^{*}\right)^{-\epsilon} Y_{t+s}\left[\frac{\Psi_{t, t+s}}{\Pi_{t, t+s}} p_{t}^{*}-\frac{\epsilon}{\epsilon-1} M C_{t+s}\left(\frac{\Psi_{t, t+s}}{\Pi_{t, t+s}} p_{t}^{*}\right)^{-\epsilon \omega}\right]=0
$$

Collecting terms and using standard definitions in NKPC derivations for the numerator and denominator, we obtain

$$
\left(p_{t}^{*}\right)^{1+\epsilon \omega}=\frac{\frac{\epsilon}{\epsilon-1} E_{t} \sum_{s=0}^{\infty}(\alpha \beta)^{s} \frac{\Lambda_{t+s}}{\Lambda_{t}}\left(\frac{\Psi_{t, t+s}}{\Pi_{t, t+s}}\right)^{-(1+\omega) \epsilon} Y_{t+s} M C_{t+s}}{E_{t} \sum_{s=0}^{\infty}(\alpha \beta)^{s} \frac{\Lambda_{t+s}}{\Lambda_{t}}\left(\frac{\Psi_{t, t+s}}{\Pi_{t, t+s}}\right)^{1-\epsilon} Y_{t+s}} \equiv \frac{C_{t}}{D_{t}} .
$$

Then, using the fact that $\Psi_{t, t}=\Pi_{t, t}=1$, we re-write $C_{t}$ as

$$
C_{t}=\frac{\epsilon}{\epsilon-1} M C_{t} Y_{t}+\alpha \beta \frac{\epsilon}{\epsilon-1} E_{t} \sum_{s=1}^{\infty}(\alpha \beta)^{s} \frac{\Lambda_{t+s}}{\Lambda_{t}}\left(\frac{\Psi_{t, t+s}}{\Pi_{t, t+s}}\right)^{-(1+\omega) \epsilon} M C_{t+s} Y_{t+s}
$$

In the next steps, we express the sum term as an expression involving $C_{t+1}$. To do so, one needs to replace all $t$-indexed terms by $t+1$-indexed terms. First, the above expression can be re-written as

$$
\begin{aligned}
C_{t}= & \frac{\epsilon}{\epsilon-1} M C_{t} Y_{t}+\alpha \beta \frac{\epsilon}{\epsilon-1} \times \\
& E_{t} \sum_{s=0}^{\infty}(\alpha \beta)^{s-1} \frac{\Lambda_{t+1}}{\Lambda_{t}} \frac{\Lambda_{t+1+(s-1)}}{\Lambda_{t+1}}\left(\frac{\Psi_{t, t+1+(s-1)}}{\Pi_{t, t+1+(s-1)}}\right)^{-(1+\omega) \epsilon} M C_{t+1+(s-1)} Y_{t+1+(s-1)} .
\end{aligned}
$$

Then, terms like $\Psi_{t, t+1+(s-1)}$ above are expressed as corresponding terms in $\Psi_{t+1, t+1+(s-1)}$ below, as follows:

$$
\begin{aligned}
\frac{\Psi_{t, t+1+(s-1)}}{\Pi_{t, t+1+(s-1)}} & =\frac{\Psi_{t, t+1+(s-1)}}{\Pi_{t, t+1+(s-1)}} \frac{\Psi_{t+1, t+1+(s-1)}}{\Psi_{t+1, t+1+(s-1)}} \frac{\Pi_{t+1, t+1+(s-1)}}{\Pi_{t+1, t+1+(s-1)}} \\
& =\frac{\Psi_{t+1, t+1+(s-1)}}{\Pi_{t+1, t+1+(s-1)}} \frac{\Psi_{t, t+1+(s-1)}}{\Psi_{t+1, t+1+(s-1)}} \frac{\Pi_{t+1, t+1+(s-1)}}{\Pi_{t, t+1+(s-1)}} .
\end{aligned}
$$

Now, using the definitions of $\Pi_{t, t+s}$ and $\Psi_{t, t+s}$ we obtain

$$
\frac{\Pi_{t+1, t+1+(s-1)}}{\Pi_{t, t+1+(s-1)}}=\frac{\frac{P_{t+1+(s-1)}}{P_{t+1}}}{\frac{P_{t+1+(s-1)}}{P_{t}}}=\frac{\frac{1}{P_{t+1}}}{\frac{1}{P_{t}}}=\frac{P_{t}}{P_{t+1}}=\frac{1}{\Pi_{t+1}}
$$

and 


$$
\frac{\Psi_{t, t+1+(s-1)}}{\Psi_{t+1, t+1+(s-1)}}=\frac{\Psi_{t, t+s}}{\Psi_{t+1, t+1+(s-1)}}=\frac{\prod_{j=0}^{s-1}\left(\Pi_{t+j}^{\rho} \bar{\Pi}_{t+j+1}^{1-\rho}\right)}{\prod_{j=0}^{s-2}\left(\Pi_{t+1+j}^{\rho} \bar{\Pi}_{t+1+j+1}^{1-\rho}\right)}
$$

Notice that

$$
\prod_{j=0}^{s-2}\left(\Pi_{t+(1+j)}^{\rho} \bar{\Pi}_{t+1+(j+1)}^{1-\rho}\right)=\prod_{j=1}^{s-1}\left(\Pi_{t+j}^{\rho} \bar{\Pi}_{t+1+j}^{1-\rho}\right)
$$

so that

$$
\frac{\Psi_{t, t+1+(s-1)}}{\Psi_{t+1, t+1+(s-1)}}=\frac{\prod_{j=0}^{s-1}\left(\Pi_{t+j}^{\rho} \bar{\Pi}_{t+j+1}^{1-\rho}\right)}{\prod_{j=1}^{s-2}\left(\Pi_{t+j}^{\rho} \bar{\Pi}_{t+1+j}^{1-\rho}\right)}=\Pi_{t}^{\rho} \bar{\Pi}_{t+1}^{1-\rho}
$$

and, finally,

$$
\frac{\Psi_{t, t+1+(s-1)}}{\Pi_{t, t+1+(s-1)}}=\frac{\Psi_{t+1, t+1+(s-1)}}{\Pi_{t+1, t+1+(s-1)}}=\frac{\Pi_{t}^{\rho} \bar{\Pi}_{t+1}^{1-\rho}}{\Pi_{t+1}} .
$$

Hence, it follows that

$$
\begin{aligned}
C_{t}= & \frac{\epsilon}{\epsilon-1} M C_{t} Y_{t}+\alpha \beta \frac{\epsilon}{\epsilon-1} \times \\
& E_{t}\left[\frac{\Lambda_{t+1}}{\Lambda_{t}}\left(\frac{\Pi_{t}^{\rho} \bar{\Pi}_{t+1}^{1-\rho}}{\Pi_{t+1}}\right)^{-(1+\omega) \epsilon} \sum_{s=0}^{\infty}(\alpha \beta)^{s} \frac{\Lambda_{t+1+s}}{\Lambda_{t+1}}\left(\frac{\Psi_{t+1, t+1+s}}{\Pi_{t+1, t+1+s}}\right)^{-(1+\omega) \epsilon} M C_{t+1+s} Y_{t+1+s}\right],
\end{aligned}
$$

which can be re-written recursively as

$$
C_{t}=\frac{\epsilon}{\epsilon-1} M C_{t} Y_{t}+\alpha \beta E_{t}\left[\frac{\Lambda_{t+1}}{\Lambda_{t}}\left(\frac{\Pi_{t}^{\rho} \bar{\Pi}_{t+1}^{1-\rho}}{\Pi_{t+1}}\right)^{-(1+\omega) \epsilon} C_{t+1}\right]
$$

Proceeding in a similar fashion with $D_{t}$, we obtain its analogous recursive representation:

$$
D_{t}=Y_{t}+\alpha \beta E_{t}\left[\frac{\Lambda_{t+1}}{\Lambda_{t}}\left(\frac{\Pi_{t}^{\rho} \bar{\Pi}_{t+1}^{1-\rho}}{\Pi_{t+1}}\right)^{1-\epsilon} D_{t+1}\right]
$$

To induce stationarity, we next define

$$
\widetilde{C}_{t} \equiv \frac{C_{t}}{Y_{t}}, \quad \widetilde{D}_{t} \equiv \frac{D_{t}}{Y_{t}},
$$


and obtain further

$$
\widetilde{C}_{t} \equiv \frac{\epsilon}{\epsilon-1} M C_{t}+\alpha \beta E_{t}\left[g_{t+1}^{\Lambda} g_{t+1}^{Y}\left(\frac{\Pi_{t}^{\rho} \bar{\Pi}_{t+1}^{1-\rho}}{\Pi_{t+1}}\right)^{-(1+\omega) \epsilon} \widetilde{C}_{t+1}\right]
$$

and

$$
\widetilde{D}_{t} \equiv 1+\alpha \beta E_{t}\left[g_{t+1}^{\Lambda} g_{t+1}^{Y}\left(\frac{\Pi_{t}^{\rho} \bar{\Pi}_{t+1}^{1-\rho}}{\Pi_{t+1}}\right)^{1-\epsilon} \widetilde{D}_{t+1}\right]
$$

where

$$
g_{t+1}^{\Lambda} \equiv \frac{\Lambda_{t+1}}{\Lambda_{t}}, \quad g_{t+1}^{Y} \equiv \frac{Y_{t+1}}{Y_{t}} .
$$

Since $\bar{\Pi}_{t}$ incorporates a random walk, we define

$$
\widetilde{\Pi}_{t} \equiv \frac{\Pi_{t}}{\bar{\Pi}_{t}}
$$

Using this, we get

$$
\begin{gathered}
\widetilde{C}_{t}=\frac{\epsilon}{\epsilon-1} M C_{t}+\alpha \beta E_{t}\left[g_{t+1}^{\Lambda} g_{t+1}^{Y}\left(\frac{\widetilde{\Pi}_{t}^{\rho}}{\widetilde{\Pi}_{t+1}}\left(g_{t+1}^{\bar{\Pi}}\right)^{-\rho}\right)^{-(1+\omega) \epsilon} \widetilde{C}_{t+1}\right], \\
\widetilde{D}_{t}=1+\alpha \beta E_{t}\left[g_{t+1}^{\Lambda} g_{t+1}^{Y}\left(\frac{\widetilde{\Pi}_{t}^{\rho}}{\widetilde{\Pi}_{t+1}}\left(g_{t+1}^{\bar{\Pi}}\right)^{-\rho}\right)^{1-\epsilon} \widetilde{D}_{t+1}\right]
\end{gathered}
$$

and their ratio,

$$
\frac{\widetilde{C}_{t}}{\widetilde{D}_{t}}=\left(\frac{P_{t}^{*}}{P_{t}}\right)^{1+\epsilon \omega}=\left(p_{t}^{*}\right)^{1+\epsilon \omega}
$$

Defining 'hat' variables to denote log-deviations of stationary variables around the drifting steady state (as in the main text), i.e., $\widehat{C}_{t} \equiv \ln \widetilde{C}_{t}=\ln \frac{C_{t}}{\bar{C}_{t}}$, we further obtain

$$
(1+\epsilon \omega) p_{t}^{*}=\widehat{C}_{t}-\widehat{D}_{t},
$$

and using the steady state restriction

$$
\widetilde{C}_{t}=\frac{1}{1-\alpha \beta g^{\Lambda} g^{Y}} \frac{\epsilon}{\epsilon-1} \widetilde{M C}_{t}
$$

we arrive at

$$
\widehat{C}_{t}=\left(1-\alpha \beta g^{\Lambda} g^{Y}\right) \widehat{M C}_{t}+\alpha \beta g^{\Lambda} g^{Y} E_{t}\left[g_{t+1}^{\Lambda}+g_{t+1}^{Y}+(1+\omega) \epsilon\left(\widehat{\Pi}_{t+1}-\rho \widehat{\Pi}_{t}+\rho \widehat{g}_{t+1}^{\bar{\pi}}\right)+\widehat{C}_{t+1}\right] .
$$


Proceeding in an analogous way for $D$, we begin from the steady state restriction

$$
\widetilde{D}_{t}=\frac{1}{1-\alpha \beta g^{\Lambda} g^{Y}}
$$

and arrive at

$$
\widehat{D}_{t}=\alpha \beta g^{\Lambda} g^{Y} E_{t}\left[g_{t+1}^{\Lambda}+g_{t+1}^{Y}-(1-\epsilon)\left(\widehat{\Pi}_{t+1}-\rho \widehat{\Pi}_{t}+\rho \widehat{g}_{t+1}^{\bar{\pi}}\right)+\widehat{D}_{t+1}\right]
$$

Forming the difference,

$$
\widehat{C}_{t}-\widehat{D}_{t}=\left(1-\alpha \beta g^{\Lambda} g^{Y}\right) \widehat{M C}_{t}+\alpha \beta g^{\Lambda} g^{Y} E_{t}\left[(1+\omega \epsilon)\left(\widehat{\Pi}_{t+1}-\rho \widehat{\Pi}_{t}+\rho \widehat{g}_{t+1}^{\bar{\pi}}\right)+\widehat{C}_{t+1}-\widehat{D}_{t+1}\right]
$$

or equivalently

$$
\widehat{p}_{t}^{*}=\frac{1-\alpha \beta g^{\Lambda} g^{Y}}{1+\epsilon \omega} \widehat{M C}_{t}+\alpha \beta g^{\Lambda} g^{Y} E_{t}\left[\left(\widehat{\Pi}_{t+1}-\rho \widehat{\Pi}_{t}+\rho \widehat{g}_{t+1}^{\bar{\pi}}\right)+\widehat{p}_{t+1}^{*}\right] .
$$

\section{B.1.2 Log-Linear Aggregate Price Level and the TVT-NKPC}

Following Cogley and Sbordone (2008), we next appropriately transform the price level to represent it as a log-linear approximation around the drifting steady state. The aggregate price level obeys

$$
P_{t}^{1-\epsilon}=\int_{0}^{1} P_{t}(j)^{1-\epsilon} d j
$$

Using backward induction, one can obtain

$$
P_{t}^{1-\epsilon}=(1-\alpha)\left(P_{t}^{*}\right)^{1-\epsilon}+\alpha\left(\Pi_{t-1}^{\rho} \bar{\Pi}_{t}^{1-\rho} P_{t-1}\right)^{1-\epsilon} .
$$

Dividing by $P_{t}$,

$$
\begin{gathered}
1=(1-\alpha)\left(p_{t}^{*}\right)^{1-\epsilon}+\alpha\left(\frac{\Pi_{t-1}^{\rho} \bar{\Pi}_{t}^{1-\rho}}{\Pi_{t}}\right)^{1-\epsilon}, \\
1=(1-\alpha)\left(p_{t}^{*}\right)^{1-\epsilon}+\alpha\left(\frac{\Pi_{t-1}^{\rho}}{\bar{\Pi}_{t}^{\rho}} \frac{\bar{\Pi}_{t}}{\Pi_{t}}\right)^{1-\epsilon} .
\end{gathered}
$$

Using the gross steady-state growth rate,

$$
g_{t}^{\bar{\pi}} \equiv \frac{\bar{\Pi}_{t}}{\bar{\Pi}_{t-1}},
$$

we further obtain

$$
\begin{aligned}
& 1=(1-\alpha)\left(p_{t}^{*}\right)^{1-\epsilon}+\alpha\left(\frac{\Pi_{t-1}^{\rho}}{\left(g_{t}^{\bar{\pi}} \bar{\Pi}_{t-1}\right)^{\rho}} \frac{1}{\widetilde{\Pi}_{t}}\right)^{1-\epsilon}, \\
& 1=(1-\alpha)\left(p_{t}^{*}\right)^{1-\epsilon}+\alpha\left(\frac{1}{\left(g_{t}^{\bar{\pi}}\right)^{\rho}} \frac{\Pi_{t-1}^{\rho}}{\bar{\Pi}_{t-1}^{\rho}} \frac{1}{\widetilde{\Pi}_{t}}\right)^{1-\epsilon},
\end{aligned}
$$




$$
1=(1-\alpha)\left(p_{t}^{*}\right)^{1-\epsilon}+\alpha\left(\frac{\widetilde{\Pi}_{t-1}^{\rho}}{\widetilde{\Pi}_{t}}\left(g_{t}^{\bar{\pi}}\right)^{-\rho}\right)^{1-\epsilon} .
$$

Transforming (17) to express it in terms of the stationary variables, we get: ${ }^{2}$

$$
1=(1-\alpha)\left(\bar{p}_{t}^{*}\right)^{1-\epsilon}\left(\widetilde{p}_{t}^{*}\right)^{1-\epsilon}+\alpha\left(g_{t}^{\bar{\pi}}\right)^{-\rho(1-\epsilon)} \widetilde{\Pi}_{t-1}^{\rho(1-\epsilon)} \widetilde{\Pi}_{t}^{-(1-\epsilon)} .
$$

Thus, in a drifting steady state at $t$, equation (18) yields:

$$
\begin{gathered}
1=(1-\alpha) \bar{p}_{t}^{*}+\alpha\left(g_{t}^{\bar{\pi}}\right)^{-\rho(1-\epsilon)}\left(g_{t}^{\bar{\pi}}\right)^{\rho(1-\epsilon)}, \\
1=1-\alpha \bar{p}_{t}^{*}+\alpha \\
\Rightarrow \bar{p}_{t}^{*}=1 .
\end{gathered}
$$

Using the 'hat' variables,

$$
\begin{gathered}
\widehat{\Pi}_{t} \equiv \ln \widetilde{\Pi}_{t}=\ln \left(\Pi_{t} / \bar{\Pi}_{t}\right)=\ln \Pi_{t}-\ln \bar{\Pi}_{t}, \\
\widehat{g}_{t}^{\bar{\pi}} \equiv \ln g_{t}^{\bar{\pi}},
\end{gathered}
$$

the log-linear approximation of (18) around the (time-varying) steady state $\bar{p}_{t}^{*}=1$ is:

$$
\widehat{p}_{t}^{*}=\frac{\alpha}{1-\alpha}\left(\widehat{\Pi}_{t}-\rho \widehat{\Pi}_{t-1}+\rho \widehat{g}_{t}^{\bar{\pi}}\right) .
$$

Employing relation (16), we finally arrive at

$$
\widehat{\Pi}_{t}-\rho \widehat{\Pi}_{t-1}+\rho \widehat{g}_{t}^{\bar{\Pi}}=\frac{(1-\alpha)\left(1-\alpha \beta g^{\Lambda} g^{Y}\right)}{\alpha(1+\epsilon \omega)} \widehat{M C}_{t}+\beta g^{\Lambda} g^{Y} E_{t}\left[\widehat{\Pi}_{t+1}-\rho \widehat{\Pi}_{t}+\rho \widehat{g}_{t+1}^{\bar{\Pi}}\right],
$$

which is equation (2) in the main text.

\section{B.2 Log-Linear Marginal Cost Condition of McKnight and Mihailov (2015)}

Linearizing equation (8) of McKnight and Mihailov (2015):

$$
\begin{aligned}
m c_{t} & =w_{t}\left(\frac{P_{t}}{P_{H, t}}\right), \\
& \Rightarrow \widehat{m c}_{t}=\widehat{w}_{t}+\left(\widehat{P}_{t}-\widehat{P}_{H, t}\right) .
\end{aligned}
$$

\footnotetext{
${ }^{2}$ Which is a generalization of eq. (26) in Appendix A of Cogley and Sbordone (2008) arising from the indexation (a fraction $1-\rho$ ) to current trend inflation we consider here in addition to the indexation (a fraction $\rho$ ) to past actual inflation as assumed by the latter authors.
} 
Linearizing the first-order condition (13) of McKnight and Mihailov (2015) but using hereafter our notation for $\bar{\omega}$ and $\sigma$ in the present paper,

$$
\begin{aligned}
w_{t} & =\frac{v_{h}\left(h_{t}\right)}{u_{c}\left(C_{t}, m_{t}\right)}, \\
& \Rightarrow \widehat{w}_{t}=\bar{\omega} \widehat{h}_{t}+\sigma \widehat{C}_{t}-\chi \widehat{m}_{t},
\end{aligned}
$$

where the coefficients are defined as

$$
\bar{\omega} \equiv \frac{\bar{h}_{t} v_{h h}}{v_{h}}>0, \quad \chi \equiv \frac{\bar{m}_{t} u_{c m}}{u_{c}}, \quad \sigma \equiv-\frac{\bar{C}_{t} u_{c c}}{u_{c}}>0 .
$$

The aggregate version of the production function (7) of McKnight and Mihailov (2015) is

$$
d_{t} Y_{t}=h_{t}
$$

where $d_{t}$ is the measure of price dispersion:

$$
d_{t} \equiv \int_{0}^{1}\left(\frac{p_{H, t}(i)}{P_{H, t}}\right)^{-\varphi} d i
$$

Combining equations (21) and (22) above yields:

$$
\widehat{m c}_{t}=\bar{\omega} \widehat{d}_{t} Y_{t}+\sigma \widehat{C}_{t}-\chi \widehat{m}_{t}+\left(\widehat{P}_{t}-\widehat{P}_{H, t}\right)
$$

Rewriting the price index, equation (2) of McKnight and Mihailov (2015):

$$
\begin{aligned}
P_{t}^{1-\epsilon} & =a P_{H, t}^{1-\epsilon}+(1-a) P_{F, t}^{1-\epsilon}, \\
& \Rightarrow\left(\frac{P_{t}}{P_{H, t}}\right)^{1-\epsilon}=a+(1-a)\left(S_{t}\right)^{1-\epsilon},
\end{aligned}
$$

after using the definition of the terms of trade $S_{t}$. Linearizing the above yields:

$$
\left(\widehat{P}_{t}-\widehat{P}_{H, t}\right)=(1-a)\left[\frac{\left(\bar{S}_{t}\right)^{1-\epsilon}}{a+(1-a)\left(\bar{S}_{t}\right)^{1-\epsilon}}\right] \widehat{S}_{t}
$$

Combining equations (23) and (24) gives the definition of $\widehat{m c}_{t}$ in a monetary open economy:

$$
\widehat{m c}_{t}=\bar{\omega} \widehat{d}_{t} Y_{t}+\sigma \widehat{C}_{t}-\chi \widehat{m}_{t}+(1-a)\left[\frac{\left(\bar{S}_{t}\right)^{1-\epsilon}}{a+(1-a)\left(\bar{S}_{t}\right)^{1-\epsilon}}\right] \widehat{S}_{t}
$$

Assuming that in the steady state $\bar{S}_{t}=1$, equation (25) simplifies to:

$$
\widehat{m c}_{t}=\bar{\omega}{\widehat{d_{t} Y_{t}}}+\sigma \widehat{C}_{t}-\chi \widehat{m}_{t}+(1-a) \widehat{S}_{t}
$$


Ignoring $\widehat{d_{t} Y_{t}}$ for the moment, nothing has changed in terms of our definition of $\widehat{m c}_{t}$ provided the linearization is performed assuming $\bar{S}_{t}=1$, which we assume as most of the open economy literature does.

The relative price dispersion measure is:

$$
d_{t}=\int_{0}^{1}\left(\frac{P_{t}^{*}}{P_{t}}\right)^{-\epsilon} d i
$$

With Calvo (1983) price-setting, the above can be written as:

$$
\begin{aligned}
d_{t}= & (1-\alpha)\left(\frac{P_{t}^{*}}{P_{t}}\right)^{-\epsilon}+\alpha(1-\alpha)\left[\frac{P_{t-1}^{*} \Pi_{t-1}^{\rho} \bar{\Pi}_{t}^{1-\rho}}{P_{t}}\right]^{-\epsilon} \\
& +\alpha^{2}(1-\alpha)\left[\frac{P_{t-2}^{*}\left(\Pi_{t-1} \Pi_{t-2}\right)^{\rho}\left(\bar{\Pi}_{t} \bar{\Pi}_{t-1}\right)^{1-\rho}}{P_{t}}\right]^{-\epsilon}+\ldots
\end{aligned}
$$

Recalling that $\Pi_{t} \equiv P_{t} / P_{t-1}$, collecting terms gives:

$$
\begin{aligned}
d_{t}= & (1-\alpha)\left(\frac{P_{t}^{*}}{P_{t}}\right)^{-\epsilon}+\alpha\left(\Pi_{t-1}^{-\epsilon}\right)^{\rho}\left(\bar{\Pi}_{t}^{-\epsilon}\right)^{1-\rho} \\
& \times \Pi_{t}^{\epsilon} \underbrace{\left[(1-\alpha)\left(\frac{P_{t-1}^{*}}{P_{t-1}}\right)^{-\epsilon}+\alpha(1-\alpha)\left(\frac{P_{t-2}^{*} \Pi_{t-2}^{\rho} \bar{\Pi}_{t-1}^{1-\rho}}{P_{t-1}}\right)^{-\epsilon}+\ldots\right]}_{\equiv d_{t-1}} .
\end{aligned}
$$

Recalling that $p_{t}^{*} \equiv P_{t}^{*} / P_{t}$, the above expression simplifies to:

$$
d_{t}=(1-\alpha)\left(p_{t}^{*}\right)^{-\epsilon}+\alpha\left(\Pi_{t-1}^{-\epsilon}\right)^{\rho}\left(\bar{\Pi}_{t}^{-\epsilon}\right)^{1-\rho} \Pi_{t}^{\epsilon} d_{t-1} .
$$

Transforming (27) to express it in terms of stationary variables:

$$
\begin{gathered}
\bar{d}_{t} \cdot \widetilde{d}_{t}=(1-\alpha)\left(\bar{p}_{t}^{*}\right)^{-\epsilon} \cdot\left(\widetilde{p}_{t}^{*}\right)^{-\epsilon}+\alpha \bar{d}_{t-1} \cdot \widetilde{d}_{t-1}\left(\bar{\Pi}_{t-1}^{-\epsilon} \cdot \widetilde{\Pi}_{t-1}^{-\epsilon}\right)^{\rho}\left(\bar{\Pi}_{t}^{-\epsilon}\right)^{-\rho} \cdot \widetilde{\Pi}_{t}^{\epsilon}, \\
\bar{d}_{t} \cdot \widetilde{d}_{t}=(1-\alpha)\left(\bar{p}_{t}^{*}\right)^{-\epsilon} \cdot\left(\widetilde{p}_{t}^{*}\right)^{-\epsilon}+\alpha \bar{d}_{t-1} \cdot \widetilde{d}_{t-1}\left(\widetilde{\Pi}_{t-1}^{-\epsilon}\right)^{\rho}\left[\left(g_{t}^{\bar{\pi}}\right)^{-\epsilon}\right]^{-\rho} \cdot \widetilde{\Pi}_{t}^{\epsilon}
\end{gathered}
$$

since $g_{t}^{\bar{\pi}} \equiv \bar{\Pi}_{t} / \bar{\Pi}_{t-1}$.

Now recall that in a time-varying steady state at $t$,

$$
\begin{gathered}
\widetilde{\Pi}_{t} \equiv \Pi_{t} / \bar{\Pi}_{t}=\bar{\Pi}_{t} / \bar{\Pi}_{t}=1, \\
\widetilde{p}_{t}^{*} \equiv p_{t}^{*} / \bar{p}_{t}^{*}=\bar{p}_{t}^{*} / \bar{p}_{t}^{*}=1, \\
\widetilde{d}_{t} \equiv d_{t} / \bar{d}_{t}=\bar{d}_{t} / \bar{d}_{t}=1,
\end{gathered}
$$

Also recall that in the steady state $\bar{p}_{t}^{*}=1$. Thus, equation (28) becomes 


$$
\bar{d}_{t}=(1-\alpha)+\alpha \bar{d}_{t-1} g_{t}^{\bar{d}},
$$

and after substituting in (29) by the definition $\bar{d}_{t} \equiv \bar{d}_{t-1} g_{t}^{\bar{d}}$, we obtain $\bar{d}_{t}=1$. Linearizing (28) around the steady state yields:

$$
\widehat{d}_{t}=-\epsilon(1-\alpha) \widehat{p}_{t}^{*}+\alpha \widehat{d}_{t-1}+\alpha \epsilon\left(\widehat{\Pi}_{t}-\rho \widehat{\Pi}_{t-1}+\rho \widehat{g}_{t}^{\bar{\pi}}\right) .
$$

Recalling that

$$
\widehat{p}_{t}^{*}=\frac{\alpha}{1-\alpha}\left(\widehat{\Pi}_{t}-\rho \widehat{\Pi}_{t-1}+\rho \widehat{g}_{t}^{\bar{\pi}}\right),
$$

equations (30) and (31) imply that $\widehat{d}_{t}=\alpha \widehat{d}_{t-1}$. As discussed by Schmitt-Grohé and Uribe (2007), $d_{t}$ has no real consequences up to first order in the stationary distribution of other endogenous variables. This means that our linear approximations to the equilibrium conditions around the steady state are justified in ignoring the variable $d_{t}$. Consequently equation (26) above simplifies to

$$
\widehat{m c}_{t}=\bar{\omega} \widehat{Y}_{t}+\sigma \widehat{C}_{t}-\chi \widehat{m}_{t}+(1-a) \widehat{S}_{t}
$$

which is eq. (7) in the main text.

\section{B.3 Alternative Types of Multistep Univariate Forecasts}

Let $y_{t} \equiv \Delta^{d} X_{t}$ denote the stationary transformation of a time series in levels or log-levels $X_{t}$, where $\Delta^{d}$ is the $d$-th difference for $X_{t}$ being integrated of order $d$, that is, $X_{t}$ is $I(d)$ and $d=\{0,1,2\}$. To forecast $X_{t}$ at horizon $h$, one has to forecast first the appropriate stationary transformation of $X_{t}, y_{t}$, at the same horizon. When $h>1$, multistep univariate forecasts can be generated in two ways, namely, by iterated autoregressive (AR) forecasts or by direct AR forecasts.

\section{B.3.1 Iterated Multistep Univariate Forecasts}

The iterated forecast always begins with the 1-step-ahead forecasting AR model for $y_{t}$, which can be written as (see Marcellino et al., 2006, pp. 502-503, whose notation we follow here)

$$
y_{t+1}=\alpha+\sum_{i=1}^{p} \phi_{i} y_{t+1-i}+\varepsilon_{t+1} .
$$

After estimating recursively by OLS the parameters in (32), the multistep forecasts of $y_{t+h}$ are obtained iteratively from

$$
\widehat{y}_{t+h \mid t}^{I}=\widehat{\alpha}+\sum_{i=1}^{p} \widehat{\phi}_{i} \widehat{y}_{t+1-i \mid t}^{I} \text {, where } \widehat{y}_{j \mid t}^{I}=y_{j} \text { for } j \leq t .
$$

Iterated forecasts of $X_{t+h}$ are then computed by accumulating the values of $\widehat{y}_{t+h \mid t}^{I}$ depending on the order of integration of $X_{t}$, as follows: 


$$
\widehat{X}_{t+h \mid t}^{I}=\left\{\begin{array}{cl}
\widehat{y}_{t+h \mid t}^{I} & \text { if } X_{t} \text { is } I(0), \\
X_{t}+\sum_{i=1}^{h} \widehat{y}_{t+i \mid t}^{I} & \text { if } X_{t} \text { is } I(1), \\
X_{t}+h \Delta X_{t}+\sum_{i=1}^{h} \sum_{j=1}^{i} \widehat{y}_{t+j \mid t}^{I} & \text { if } X_{t} \text { is } I(2) .
\end{array}\right.
$$

\section{B.3.2 Direct Multistep Univariate Forecasts}

The direct estimates of the parameters are the recursive minimizers of the $h$-step-ahead criterion function. Accordingly, the parameters are estimated by OLS from the following direct forecasting AR model (again, we follow Marcellino et al., 2006, p. 503):

$$
y_{t+h}^{h}=\beta+\sum_{i=1}^{p} \rho_{i} y_{t+1-i}+\varepsilon_{t+h},
$$

where now

$$
y_{t+h}^{h}=\left\{\begin{array}{cc}
X_{t+h} & \text { if } X_{t} \text { is } I(0), \\
X_{t+h}-X_{t} & \text { if } X_{t} \text { is } I(1), \\
\sum_{i=1}^{h} \sum_{j=1}^{i} \Delta^{2} X_{t+j}=X_{t+h}-X_{t}-h \Delta X_{t} & \text { if } X_{t} \text { is } I(2) .
\end{array}\right.
$$

The direct estimator of the parameters is obtained by recursive OLS estimation of (35) where data through period $t$ are used, so that the last observation includes $y_{t}^{h}$ on the left-hand side of the regression. Then, the direct multistep forecasts of $y_{t+h}^{h}$ are given by

$$
\widehat{y}_{t+h \mid t}^{D, h}=\widehat{\beta}+\sum_{i=1}^{p} \widehat{\rho}_{i} y_{t+1-i} .
$$

By analogy with the iterated multistep AR forecasts in (34), direct multistep AR forecasts of $X_{t+h}$ are recovered from $\widehat{y}_{t+h \mid t}^{D, h}$ depending on the order of integration of $X_{t}$ :

$$
\widehat{X}_{t+h \mid t}^{D}=\left\{\begin{array}{cl}
\widehat{y}_{t+h \mid t}^{D, h} & \text { if } X_{t} \text { is } I(0), \\
X_{t}+\widehat{y}_{t+h \mid t}^{D, h} & \text { if } X_{t} \text { is } I(1) \\
X_{t}+h \Delta X_{t}+\widehat{y}_{t+h \mid t}^{D, h} & \text { if } X_{t} \text { is } I(2)
\end{array}\right.
$$




\section{Additional Results}

This Appendix C provides additional results in terms of stationarity tests (as discussed in the main text) and robustness checks (3 tables) and graphical illustrations (19 figures), some of which are referred to in the main text, with minimal comments that follow.

\section{C.1 Stationarity Tests and Further Robustness Checks}

Table 1 reports the detailed results from the stationarity tests on inflation and its components and drivers in the EA and the US, most of which were discussed in section 3 of the main text.

As a further check of robustness of our particular - but typical in macroeconomic theory and empirics - detrending choice, Figure 1 presents alternative estimates for US trend inflation in the recent literature, such as Stock and Watson (2007) and Chan et al. (2013, 2016, 2017), all based on the CPI measure of the aggregate price level (for the EA we have not found a similar comparison). While trend inflation measures differ in time patterns and - especially volatility and smoothness - we can infer from this figure that our inflation trend measure based on Hodrick-Prescott (1997) filtering falls quite in the middle of these alternative estimates, in terms of dynamics and fluctuations. This is reassuring in the sense that our 'first pass' here based on one-sided Hodrick-Prescott detrending (to resemble real-time prediction) may be robust to alternative extraction of trend inflation, although we leave this issue (that could potentially improve our forecasting procedure) for further investigation.

Tables 2 and 3 in the main text reported the key results in evaluating the predictive performance of our TVT-NKPC forecasting procedure, where the statistical significance in the Diebold-Mariano (1995) test based on the null of equal predictive accuracy was indicated by the modified DM statistic, to correct for small sample bias, as proposed by Harvey et al. (1997). Since our forecasting evaluation period is not that small, in effect, ranging from 44 (if the horizon is 20 quarters ahead) to 64 (if the horizon is 1 quarter ahead) quarters, we here also present a version of the same results in appendix tables 2 and 3 in this section, but now applying the original DM statistic in judging about statistical significance.

It can be seen, comparing the respective significance levels in tables 2 and 3 in this Appendix $\mathrm{C}$ with tables 2 and 3, respectively, already reported in the main text that such an alteration, which ignores the small sample size correction, results in even more favorable outcomes regarding the predictive accuracy of our method. More precisely, the final conclusions in the main text and its abstract can now be slightly modified, in the following sense. Based on the original DM statistic, we conclude that our TVT-NKPC forecasting procedure significantly outperforms the conventional random walk benchmark at all horizons (remaining statistically indistinguishable only at 4 quarters). Moreover, it also outperforms quantitatively, by about 10 percentage points beyond the short run of 1 and 4 quarters, the agnostic Atkeson-Ohanian (2001) benchmark, and in a statistically significant fashion in the US at the medium run of 8 and 12 quarters. 


\begin{tabular}{ccc}
\hline \hline Generalized NKPC component & ADF test t-stat pv & KPSS test LM stat \\
\hline & & $1 \% \mathrm{cv}=0.7390$ \\
& & $5 \% \mathrm{cv}=0.4630$ \\
& & $10 \% \mathrm{cv}=0.3470$ \\
\hline EA: full (adjusted) sample & 179 to 182 observations & 183 to 184 observations \\
\hline EA inflation & 0.6647 & 0.5998 \\
Cycle of EA inflation & 0.0000 & 0.0191 \\
Cycle of EA MOE real marginal cost & 0.0000 & 0.0201 \\
Cycle of EA real unit labor cost & 0.0000 & 0.0214 \\
EA (log-)trend inflation & 0.0558 & 1.4018 \\
Growth rate of EA trend inflation & 0.0402 & 0.2157 \\
\hline US: full (adjusted) sample & 181 to 182 observations & 183 to 184 observations \\
\hline US inflation & 0.1956 & 0.5639 \\
Cycle of US inflation & 0.0000 & 0.0181 \\
Cycle of US real marginal cost & 0.0001 & 0.0204 \\
Cycle of US real unit labor cost & 0.0000 & 0.0214 \\
US (log-)trend inflation & 0.2040 & 1.2243 \\
Growth rate of US trend inflation & 0.0474 & 0.1661 \\
\hline \hline
\end{tabular}

Table 1: Stationarity Tests for the Components and Drivers of the TVT-NKPC

Note: Separation of the cyclical component from the trend component of the respective time series has been obtained in the whole (adjusted) sample by applying a two-sided Hodrick-Prescott (1997) filter. The null of the Augmented Dickey-Fuller (ADF) test (Dickey and Fuller, 1979; Said and Dickey, 1984) is nonstationarity (unit root): MacKinnon (1996) one-sided probability values (pv) for the ADF test t-statistic are provided in the table; the lag length is automatic, based on the Schwarz (1978) Information Criterion (SIC), with a maximum lag set at 13 quarters; the reported results are for the ADF specification that includes a constant. The null of the Kwiatkowski-Phillips-Schmidt-Shin (KPSS, 1992) test is stationarity: asymptotic critical values (cv) for the KPSS test LM-statistic at conventional levels are provided in the table; the reported results are for the KPSS specification that includes a constant; the bandwidth is selected automatically, based on Andrews (1991) and using Bartlett (1950) kernel. 


\begin{tabular}{|c|c|c|c|c|c|c|}
\hline Forecasting evaluation period & \multicolumn{6}{|c|}{ 2000:1-2015:4 } \\
\hline Forecast horizon, quarters & 1 & 4 & 8 & 12 & 16 & 20 \\
\hline \multicolumn{7}{|c|}{ Panel A: Root MSFE } \\
\hline \multicolumn{7}{|c|}{ Theory-Based TVT-NKPC Procedures of Inflation Forecasting } \\
\hline MOE RMC (roll) & 0.729 & 0.884 & 0.912 & 0.829 & 0.745 & 0.883 \\
\hline MOE RMC $(1$ & 0.706 & 0.879 & 0.914 & 0.842 & 0.759 & 0.838 \\
\hline RULC RMC (r & 0.751 & 0.873 & 0.892 & 0.816 & 0.739 & 0.880 \\
\hline RULC & 0.765 & 0.879 & 0.892 & 0.822 & 0.747 & 0.833 \\
\hline \multicolumn{7}{|c|}{ Agnostic Univariate Benchmarks of Inflation Forecasting } \\
\hline RW Forecast & 0.909 & 0.885 & 1.038 & 1.001 & 0.996 & 1.092 \\
\hline AO (Pseudo-RW) Forecast & 0.669 & 0.815 & 0.913 & 0.894 & 0.819 & 0.921 \\
\hline \multicolumn{7}{|c|}{ Panel B: Theil U-stat to RW $\equiv$ root MSFE of TVT-NKPC w.r.t. RW forecast } \\
\hline MOE RMC (roll) & $0.802^{* * *}$ & 1.000 & 0.879 & 0.821 & $0.748^{* *}$ & 0.808 \\
\hline MOE RMC $(1$ & $0.776^{* * *}$ & 0.993 & 0.881 & 0.834 & $0.762^{* *}$ & $0.767^{* *}$ \\
\hline RULC RMC (1 & $0.826^{* *}$ & 0.987 & $0.859^{*}$ & $0.809^{*}$ & $0.742^{* *}$ & 0.805 \\
\hline RULC RMC (rec) & $0.842^{* *}$ & 0.993 & $0.860^{*}$ & $0.814^{*}$ & $0.751^{* *}$ & $0.763^{* *}$ \\
\hline \multicolumn{7}{|c|}{ Panel C: Theil U-stat to AO $\equiv$ root MSFE of TVT-NKPC w.r.t. AO forecast } \\
\hline MOE RMC (roll) & $1.090^{* *}$ & 1.086 & 0.999 & 0.927 & 0.909 & 0.958 \\
\hline MOE RMC ( & 1.053 & 1.078 & 1.001 & 0.942 & 0.926 & 0.910 \\
\hline RULC RMC & $1.121^{* * *}$ & $1.072^{*}$ & 0.977 & 0.914 & 0.901 & 0.955 \\
\hline RULC RMC (rec) & $1.143^{* * *}$ & $1.079 * *$ & 0.977 & 0.920 & 0.912 & 0.905 \\
\hline
\end{tabular}

Table 2: Predictive Performance of TVT-NKPC Forecasts in the EA Data - DM Test

Note: See the note below Table 2 in the main text. Differently here, the original Diebold-Mariano (1995) t-statistic is used, and is again computed with p-values using Newey-West (1987) HAC standard errors; it tests the null of no significant difference in the forecast accuracy of two compared (non-nested) models; statistical significance of the (one-sided) test is shown at conventional levels: ${ }^{* * *} 1 \%,{ }^{* *} 5 \%$, and $* 10 \%$. These results are robust to applying instead the modified DM t-statistic test of Harvey et al. (1997) that corrects for small-sample bias - see Table 2 in the main text. 


\begin{tabular}{|c|c|c|c|c|c|c|}
\hline Forecasting evaluation period & \multicolumn{6}{|c|}{ 2000:1-2015:4 } \\
\hline Forecast horizon, quarters & 1 & 4 & 8 & 12 & 16 & 20 \\
\hline \multicolumn{7}{|c|}{ Panel A: Root MSFE } \\
\hline \multicolumn{7}{|c|}{ Theory-Based TVT-NKPC Procedures of Inflation Forecasting } \\
\hline MOE RMC (roll) & 0.854 & 1.156 & 1.165 & 1.249 & 1.255 & 1.662 \\
\hline MOE RMC (r & 0.986 & 1.125 & 1.438 & 1.370 & 1.291 & 1.144 \\
\hline RULC RMC & 14 & 1.041 & 1.100 & 1.229 & 1.286 & 1.655 \\
\hline RULC & 0.922 & 1.036 & 1.127 & 1.227 & 1.261 & 1.179 \\
\hline \multicolumn{7}{|c|}{ Agnostic Univariate Benchmarks of Inflation Forecasting } \\
\hline RW Forecast & 0.970 & 1.127 & 1.333 & 1.360 & 1.470 & 1.447 \\
\hline AO (Pseudo-RW) Forecast & 0.868 & 1.027 & 1.222 & 1.344 & 1.376 & 1.251 \\
\hline \multicolumn{7}{|c|}{ Panel B: Theil U-stat to RW $\equiv$ root MSFE of TVT-NKPC w.r.t. RW forecast } \\
\hline MOE RMC (roll) & $0.881^{*}$ & 1.034 & 0.874 & 0.918 & 0.854 & 1.149 \\
\hline MOE RMC (rec) & 1.016 & 0.998 & 1.079 & 1.007 & 0.878 & $0.790^{\diamond}$ \\
\hline RULC I & 0.942 & 0.924 & $0.826^{*}$ & $0.903^{*}$ & 0.875 & 1.114 \\
\hline RULC RMC (rec) & 0.950 & 0.919 & $0.845^{*}$ & $0.902^{*}$ & $0.858^{*}$ & 0.812 \\
\hline \multicolumn{7}{|c|}{ Panel C: Theil U-stat to AO $\equiv$ root MSFE of TVT-NKPC w.r.t. AO forecast } \\
\hline MOE RMC (roll) & 0.984 & $1.134^{* *}$ & 0.953 & 0.929 & 0.912 & $1.329^{*}$ \\
\hline MOE RMC (rec) & $1.135^{* * *}$ & 1.096 & $1.177^{*}$ & 1.020 & 0.938 & 0.914 \\
\hline RULC RMC (roll) & 1.053 & 1.014 & $0.901^{* *}$ & $0.914^{* *}$ & 0.935 & $1.323^{*}$ \\
\hline RULC RMC (rec) & 1.061 & 1.001 & $0.922^{* *}$ & $0.913^{*}$ & 0.916 & 0.943 \\
\hline
\end{tabular}

Table 3: Predictive Performance of TVT-NKPC Forecasts in the US Data - DM Test

Note: See the note below Table 2 in this supplementary online appendix, as well as Table 3 in the main text for the corresponding US results when applying the modified DM t-statistic instead of the original DM t-statistic here. $\diamond$-superscript denotes that the p-value of the DM t-statisitic in the respective cell of the table above is just marginal at the $10 \%$ sigificance level here, 0.1092 . 


\section{C.2 Further Graphical Illustrations}

The following figures illustrate various aspects and properties of our data set, inflation forecasts (for the EA and the US at the examined horizons) or some of their central components, as implied by our TVT-NKPC inflation forecasting procedure.

\section{References}

[1] Andrews, D.W.K., 1991. Heteroskedasticity and autocorrelation consistent covariance matrix estimation. Econometrica 59, 817-858.

[2] Atkeson, A., Ohanian, L.E., 2001. Are Phillips curves useful for forecasting inflation? Quarterly Review, Federal Reserve Bank of Minneapolis 25, 2-11.

[3] Bartlett, M.S., 1950. Tests of significance in factor analysis. British Journal of Mathematical and Statistical Psychology 3, 77-85.

[4] Calvo, G., 1983. Staggered prices in a utility maximizing framework. Journal of Monetary Economics 12, 383-398.

[5] Chan, J.C.C., Clark, T.E., Koop, G., 2018. A new model of inflation, trend inflation, and long-run inflation expectations. Journal of Money, Credit and Banking 50, 5-53.

[6] Chan, J.C.C., Koop, G., Potter, S.M., 2013. A new model of trend inflation. Journal of Business and Economic Statistics 31, 94-106.

[7] Chan, Joshua C.C., Koop, G., Potter, S.M., 2016. A bounded model of time variation in trend inflation, NAIRU and the Phillips curve. Journal of Applied Econometrics 31, $551-565$.

[8] Cogley, T., Sbordone, A., 2008. Trend inflation, indexation, and inflation persistence in the new Keynesian Phillips curve. American Economic Review 98, 2101-2126.

[9] Dickey, D.A., Fuller, W.A., 1979. Distribution of the estimators for autoregressive time series with a unit root. Journal of the American Statistical Association 74, 427-431.

[10] Diebold, F.X., Mariano, R.S., 1995. Comparing predictive accuracy. Journal of Business and Economic Statistics 13, 253-263.

[11] Fagan, G., Henry, J., Mestre, R., 2005. An area-wide model for the euro area. Economic Modelling 22, 39-59.

[12] Harvey, D., Leybourne, S., Newbold, P., 1997. Testing the equality of prediction mean squared errors. International Journal of Forecasting 13, 281-291.

[13] Hodrick, R.J., Prescott, E.C., 1997. Postwar US business cycles: An empirical investigation. Journal of Money, Credit and Banking 29, 1-16. 
[14] Kwiatkowski, D., Phillips, P.C.B., Schmidt, P., Shin, Y., 1992. Testing the null hypothesis of stationarity against the alternative of a unit root. Journal of Econometrics 54, 159-178.

[15] MacKinnon, J., 1996. Numerical distribution functions for unit root and cointegration tests. Journal of Applied Econometrics 11, 601-618.

[16] Marcellino, M., Stock, J.H., Watson, M.W., 2006. A comparison of direct and iterated multistep AR methods for forecasting macroeconomic time series. Journal of Econometrics $135,499-526$.

[17] McKnight, S., Mihailov, A., 2015. Do real balance effects invalidate the Taylor principle in closed and open economies? Economica 82, 938-975.

[18] Newey, W.K., West, K.D., 1987. A simple, positive semi-definite, heteroskedasticity and autocorrelation consistent covariance matrix. Econometrica 55, 703-708.

[19] Said, S.E., Dickey, D.A., 1984. Testing for unit roots in autoregressive moving-average models with unknown order. Biometrika 71, 599-607.

[20] Schmitt-Grohé, S., Uribe, M., 2007. Optimal simple and implementable monetary and fiscal rules. Journal of Monetary Economics 54, 1702-1725.

[21] Schwarz, G.E., 1978. Estimating the dimension of a model. Annals of Statistics 6, 461-464.

[22] Stock, J.H., Watson, M.W., 2007. Why has US inflation become harder to forecast? Journal of Money, Credit and Banking 39, 3-34. 

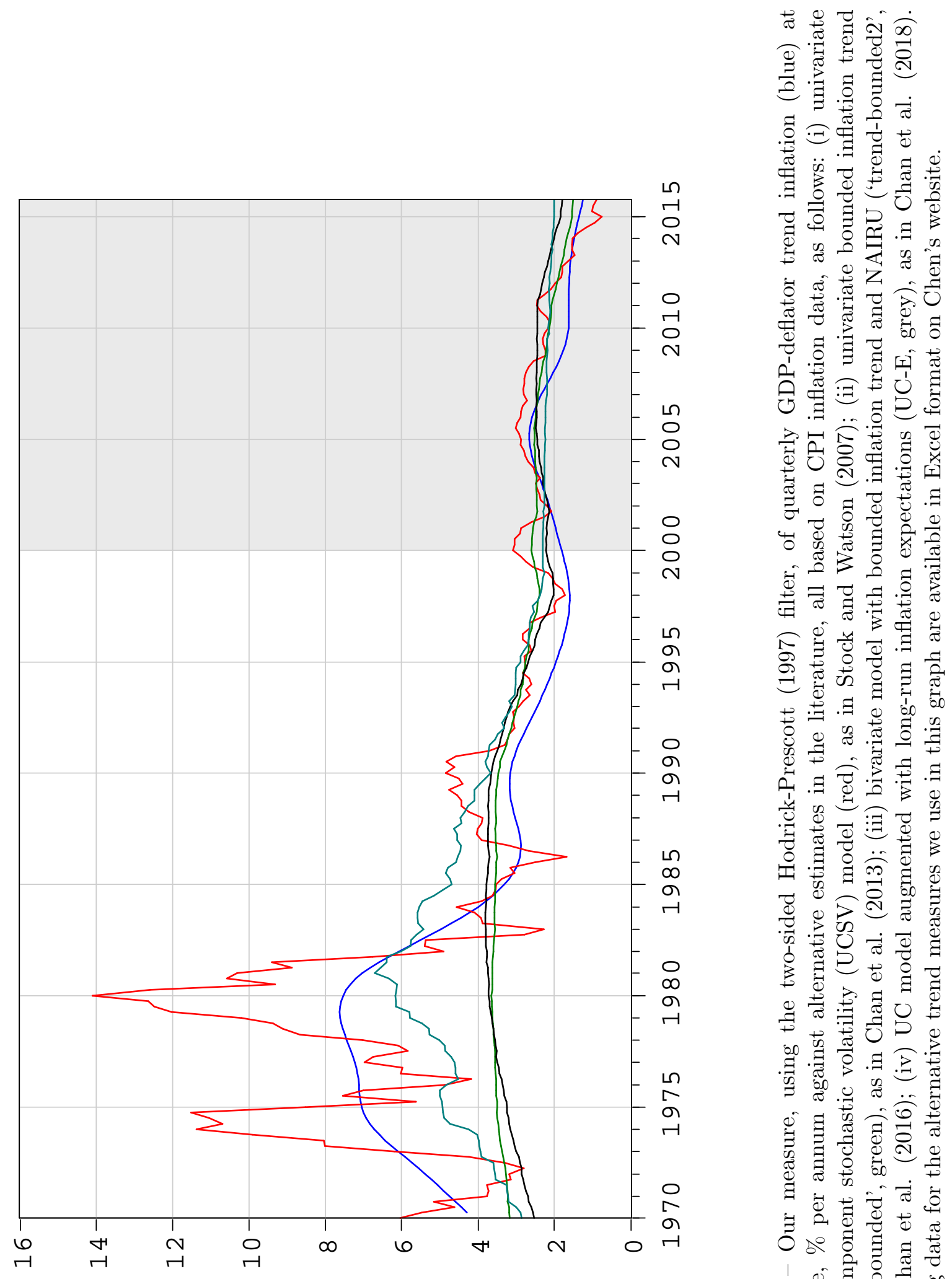

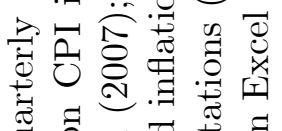

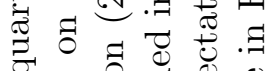

पे ठ্

ษ

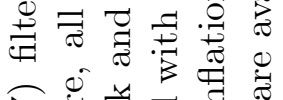

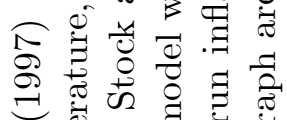

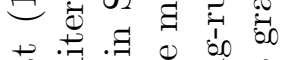

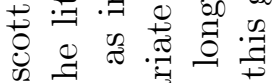

ค.

它

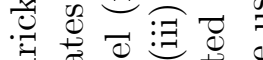

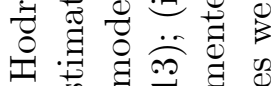

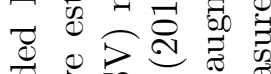

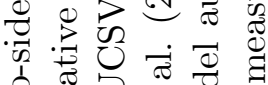

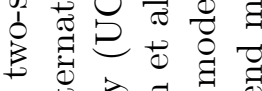

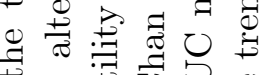

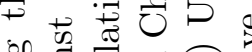

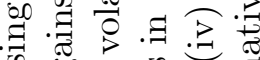

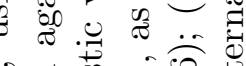

๑

ज़

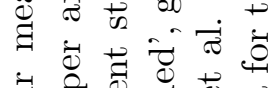

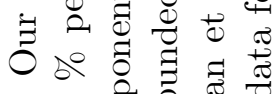

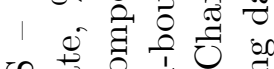

ص

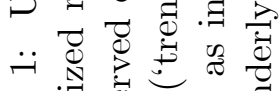

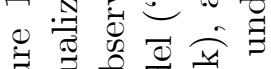

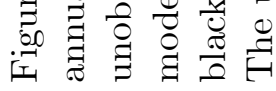




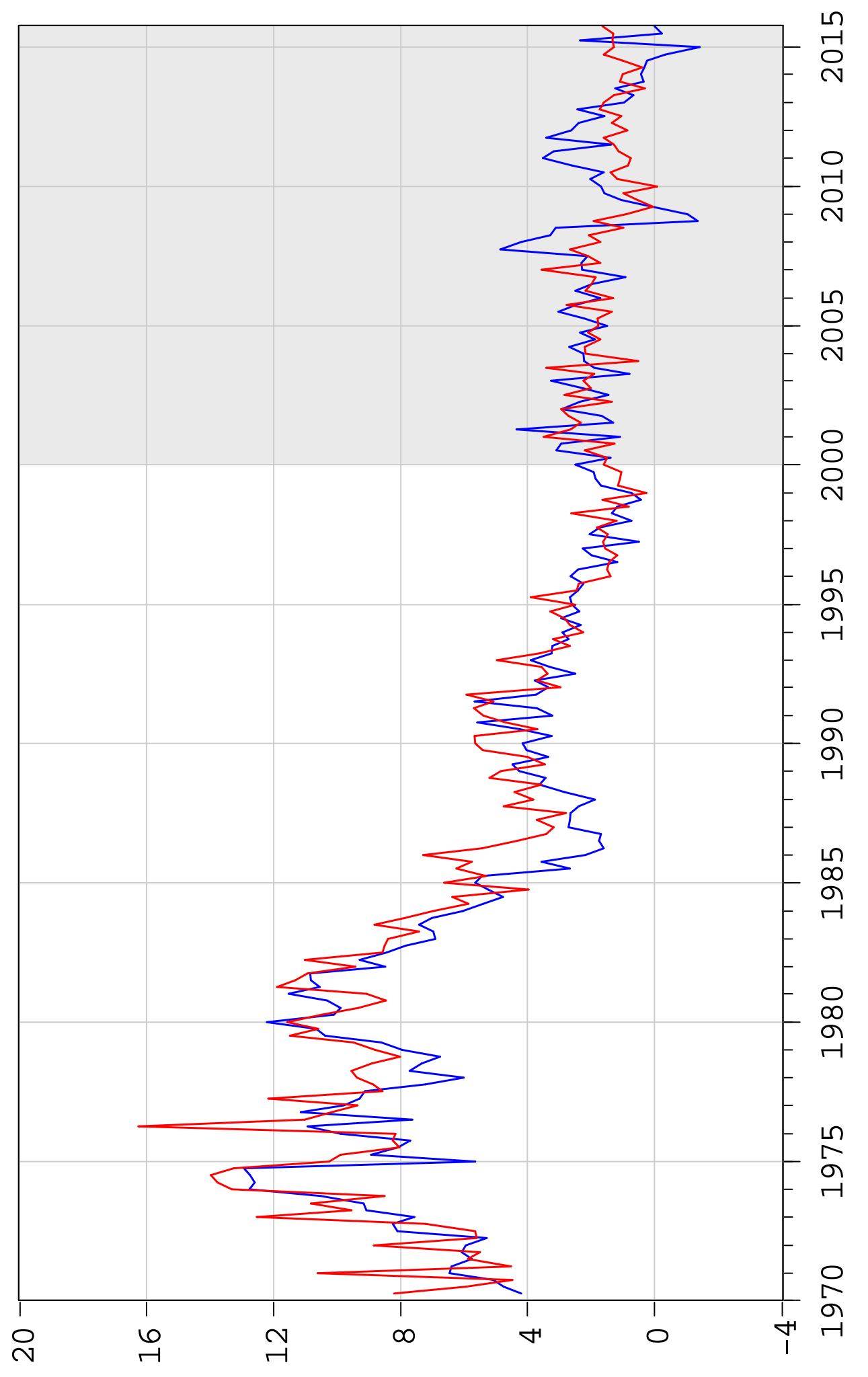




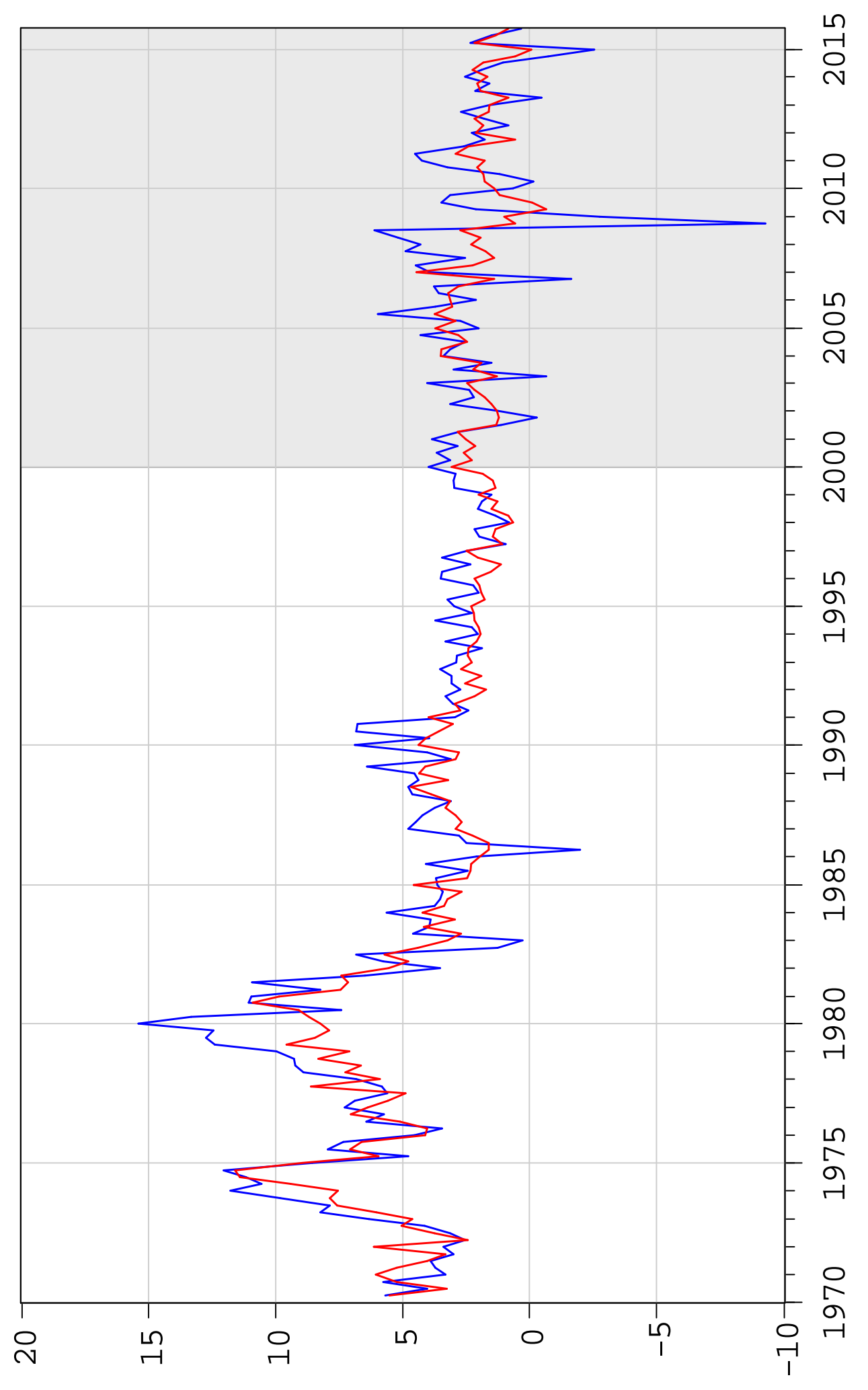




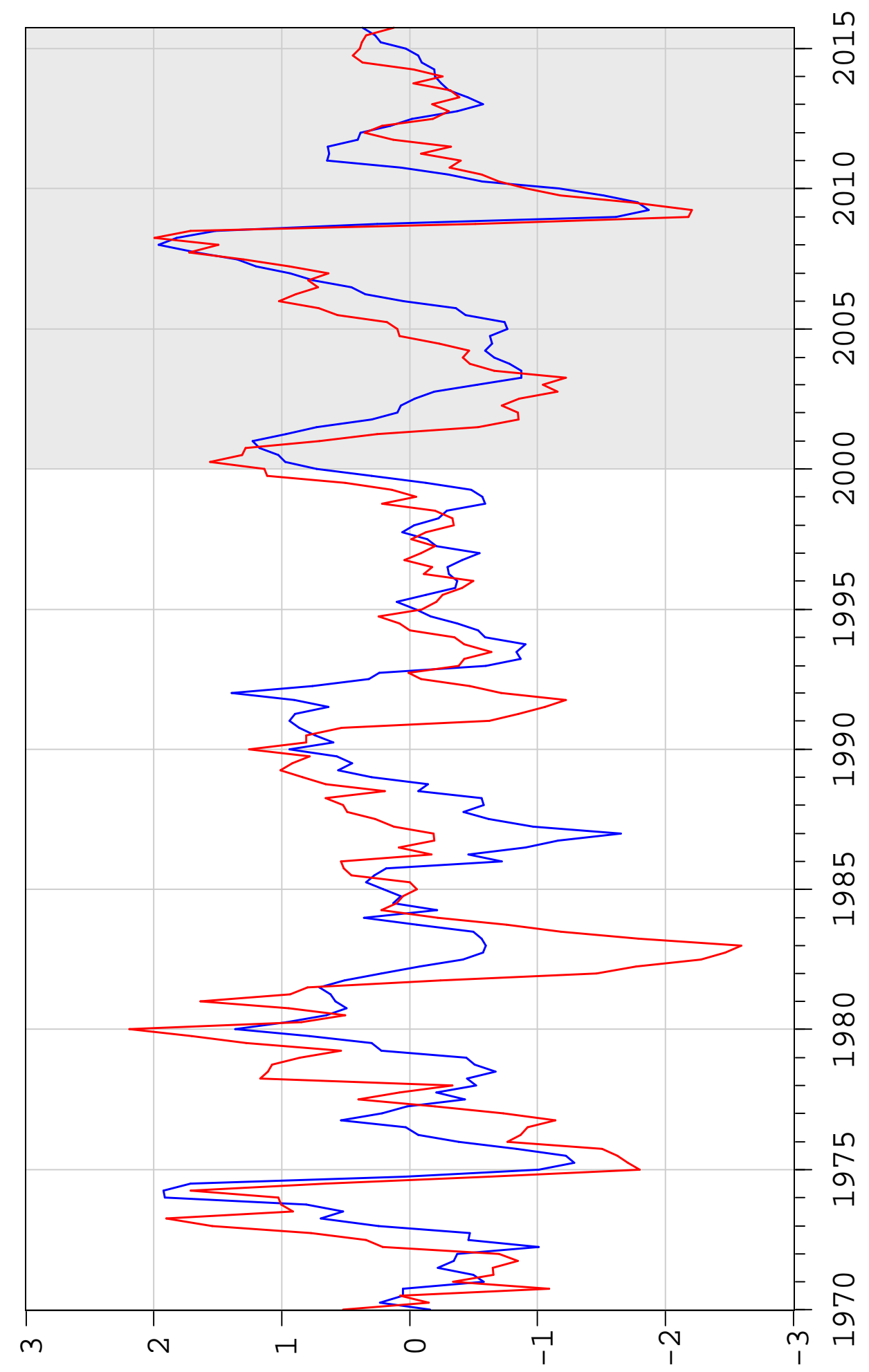




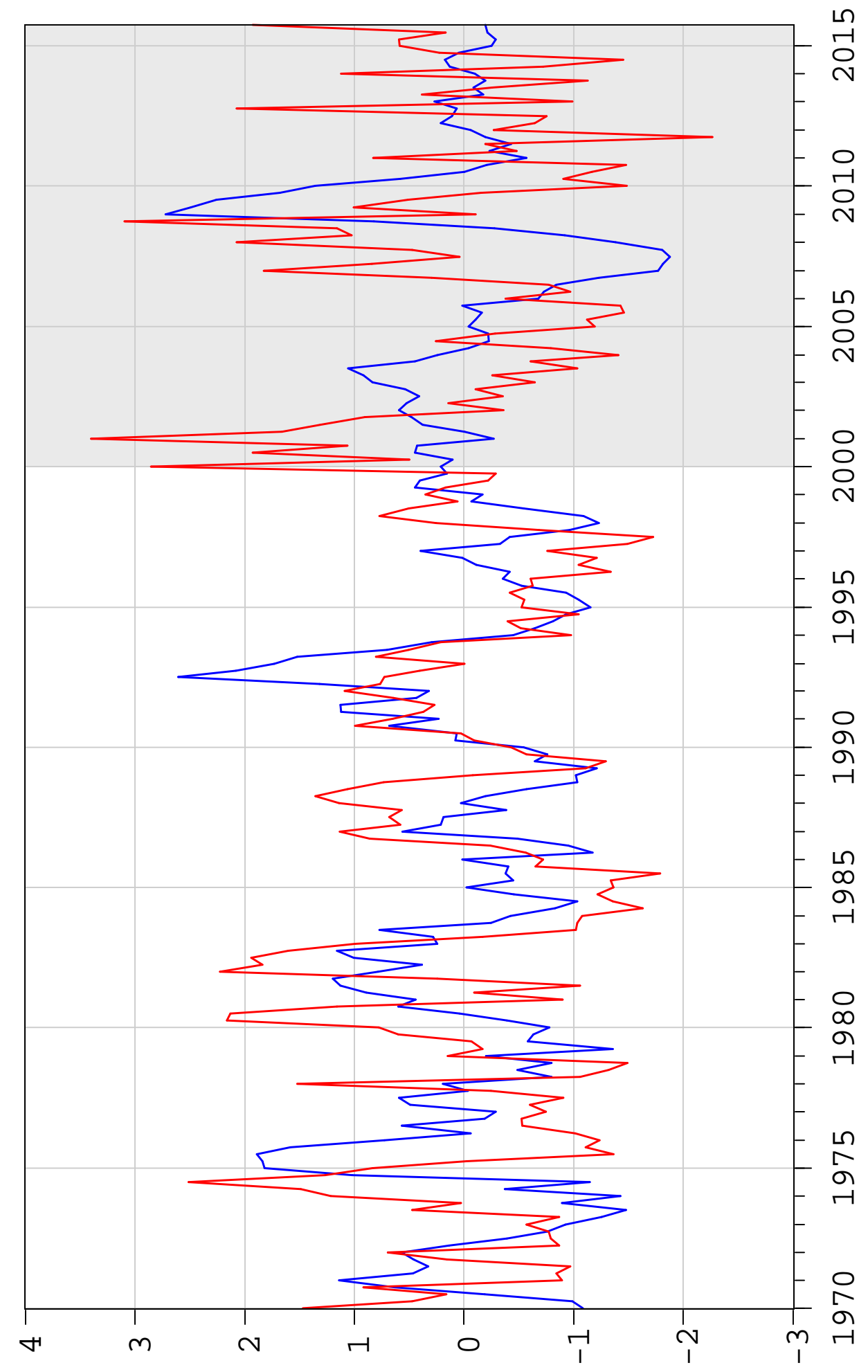



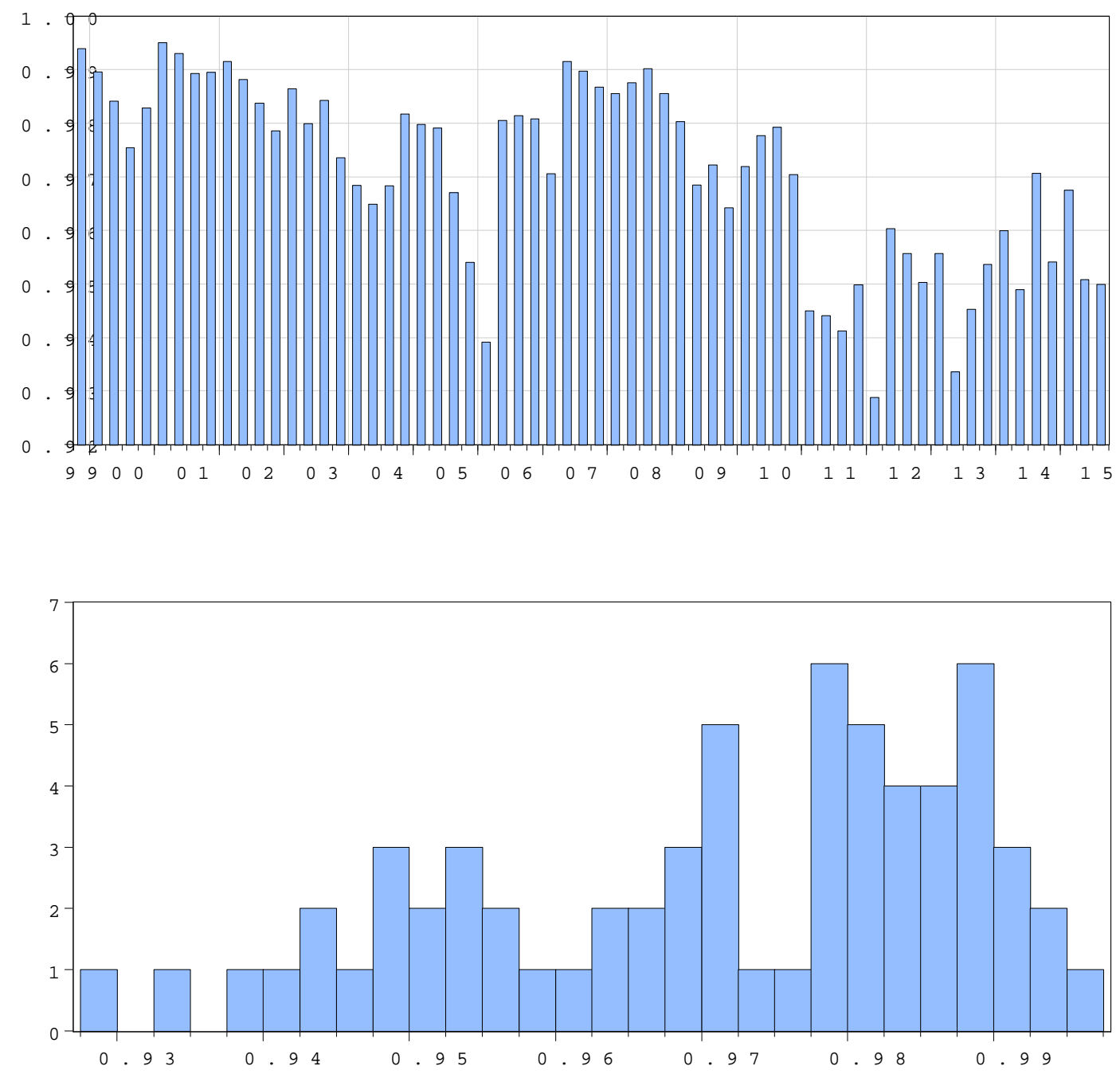

Figure 6: EA - Recursively Re-estimated Persistence $\left(g_{t}^{\bar{\Pi}}\right)$ of Trend Inflation (fixed rolling window of 113 quarters), plot (top graph) and histogram (bottom graph); 1999Q4-2015Q3, 64 observations; mean 0.971, median 0.975, max 0.995, min 0.929, SD 0.017 

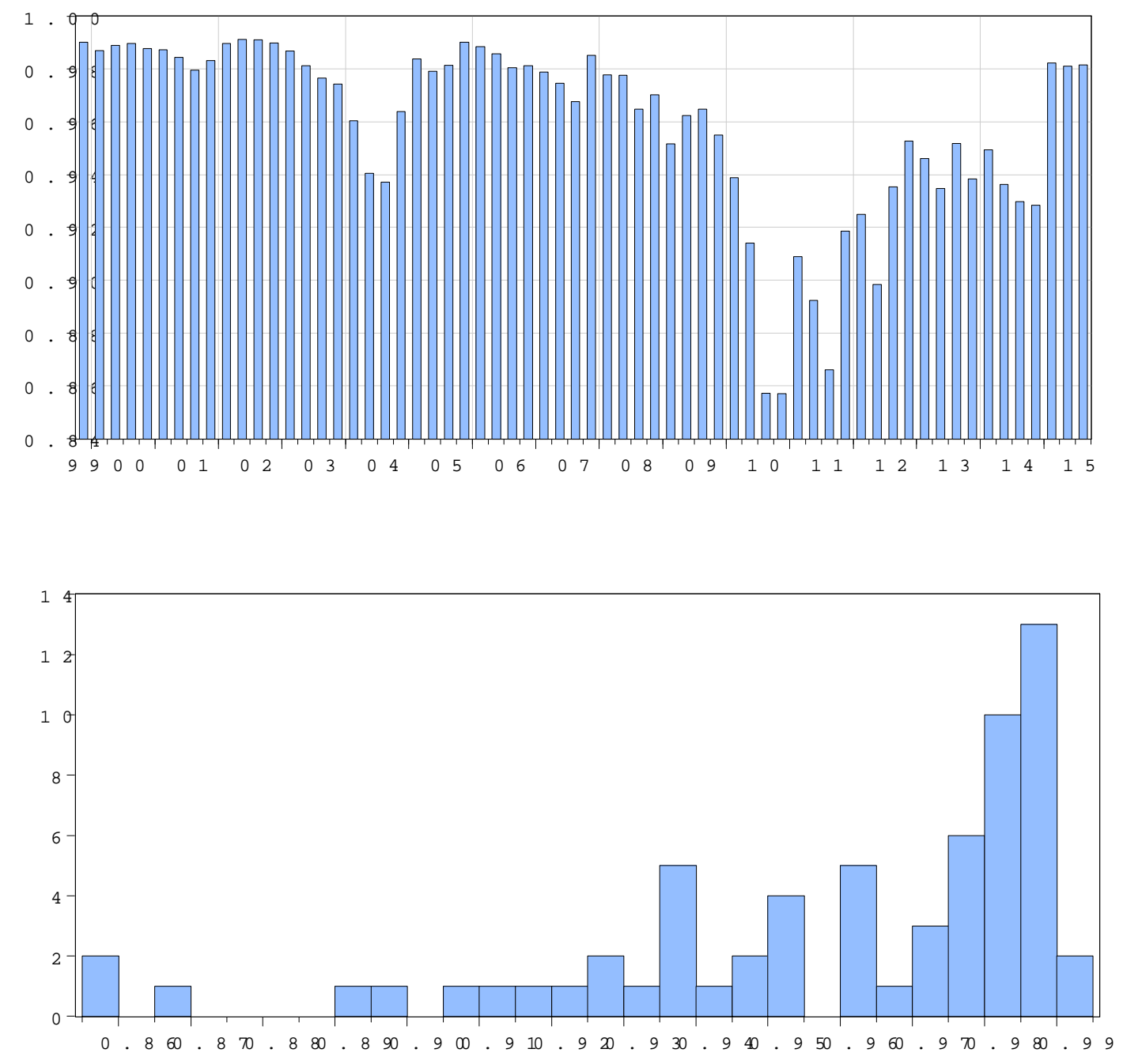

Figure 7: US - Recursively Re-estimated Persistence $\left(g_{t}^{\bar{\Pi}}\right)$ of Trend Inflation (fixed rolling window of 113 quarters), plot (top graph) and histogram (bottom graph); 1999Q4-2015Q3, 64 observations; mean 0.959, median 0.974, max 0.991, min 0.857, SD 0.034 

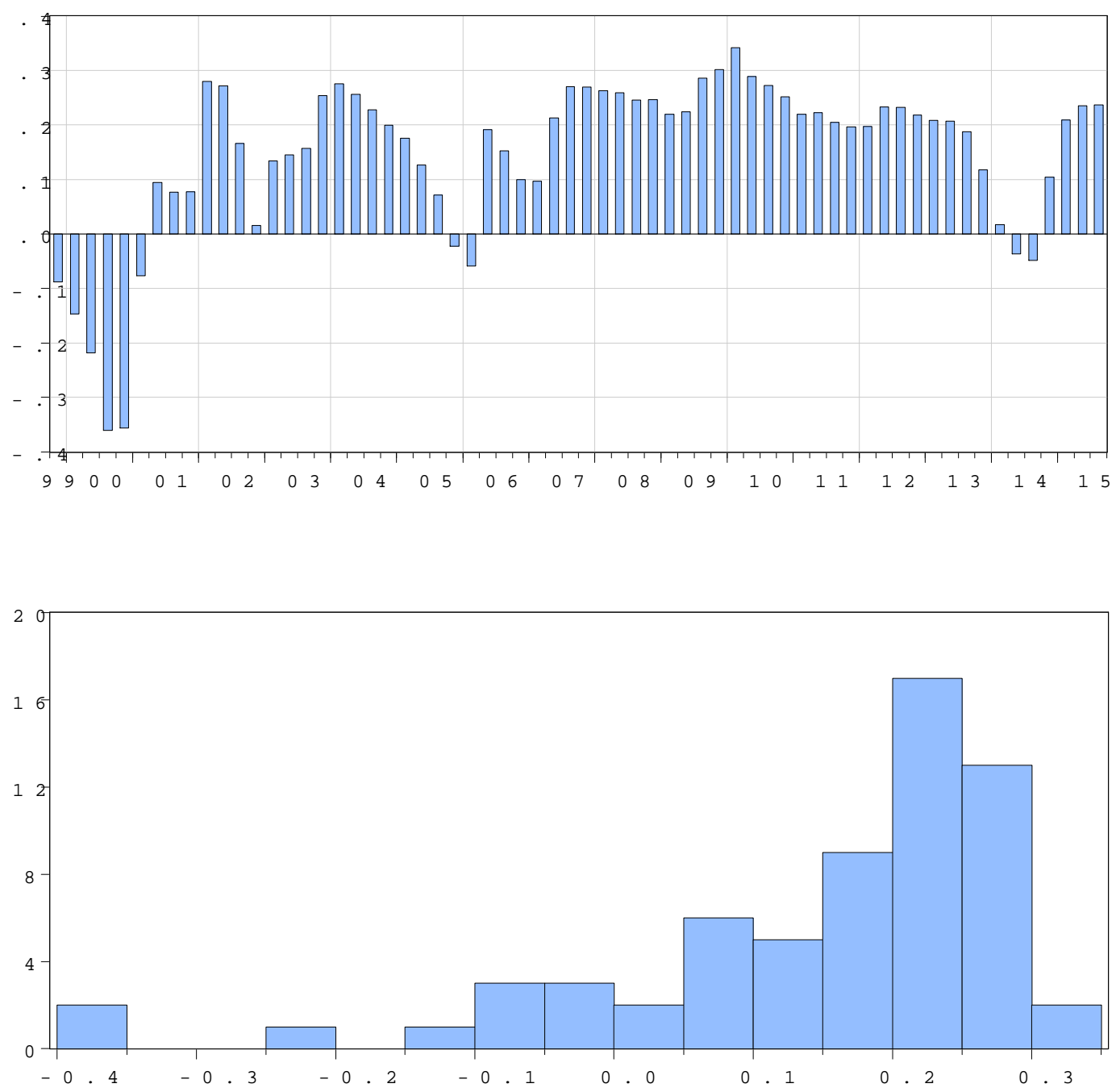

Figure 8: EA - Recursively Re-estimated Persistence $(\theta)$ of Trend Inflation Growth (fixed rolling window of 113 quarters), plot (top graph) and histogram (bottom graph); 1999Q4-2015Q3, 64 observations; mean 0.146, median 0.202, max 0.341, min -0.361, SD 0.150 

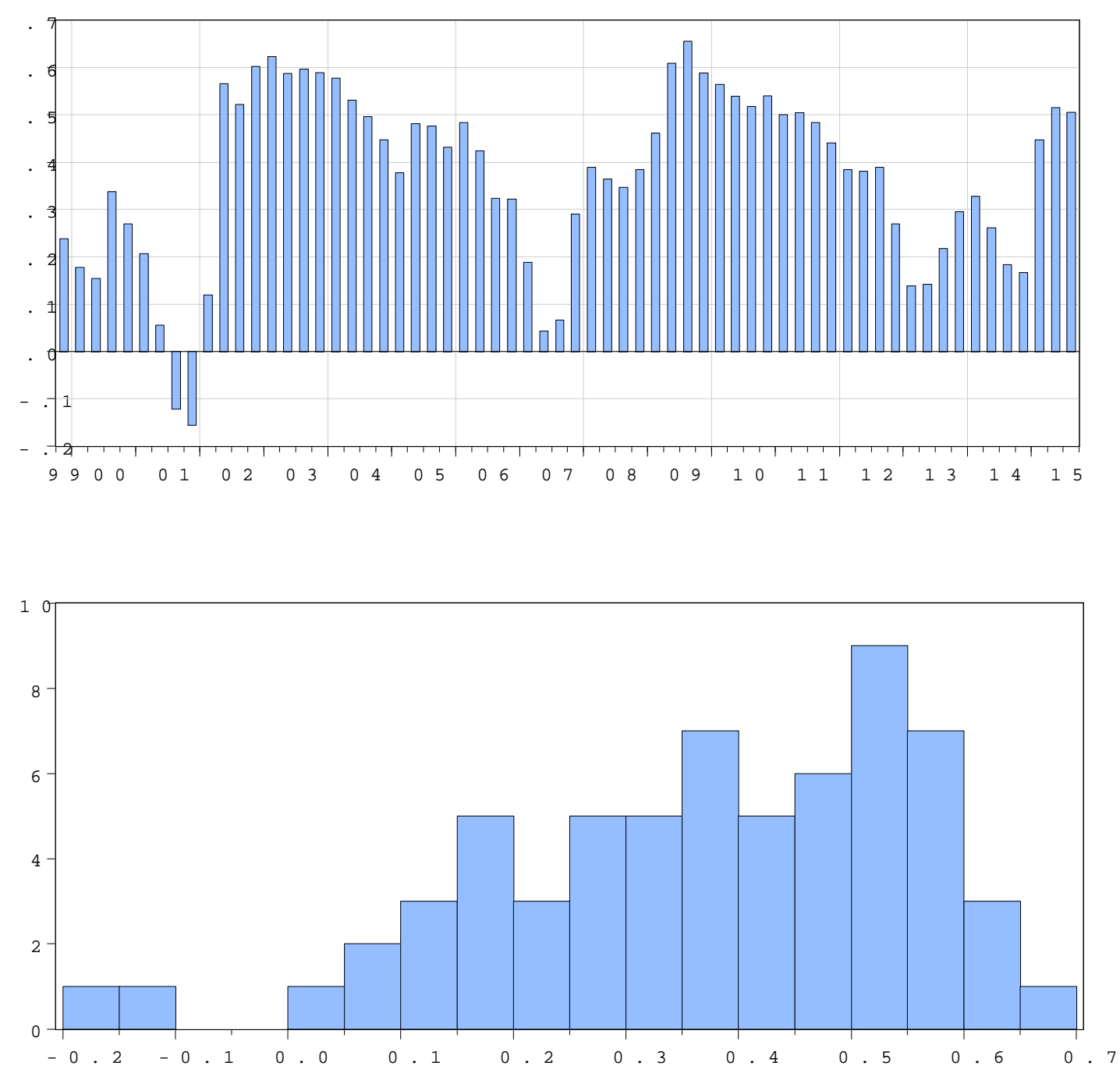

Figure 9: US - Recursively Re-estimated Persistence $(\theta)$ of Trend Inflation Growth (fixed rolling window of 113 quarters), plot (top graph) and histogram (bottom graph); 1999Q4-2015Q3, 64 observations; mean 0.373, median 0.389, max 0.655, min -0.156, SD 0.186 

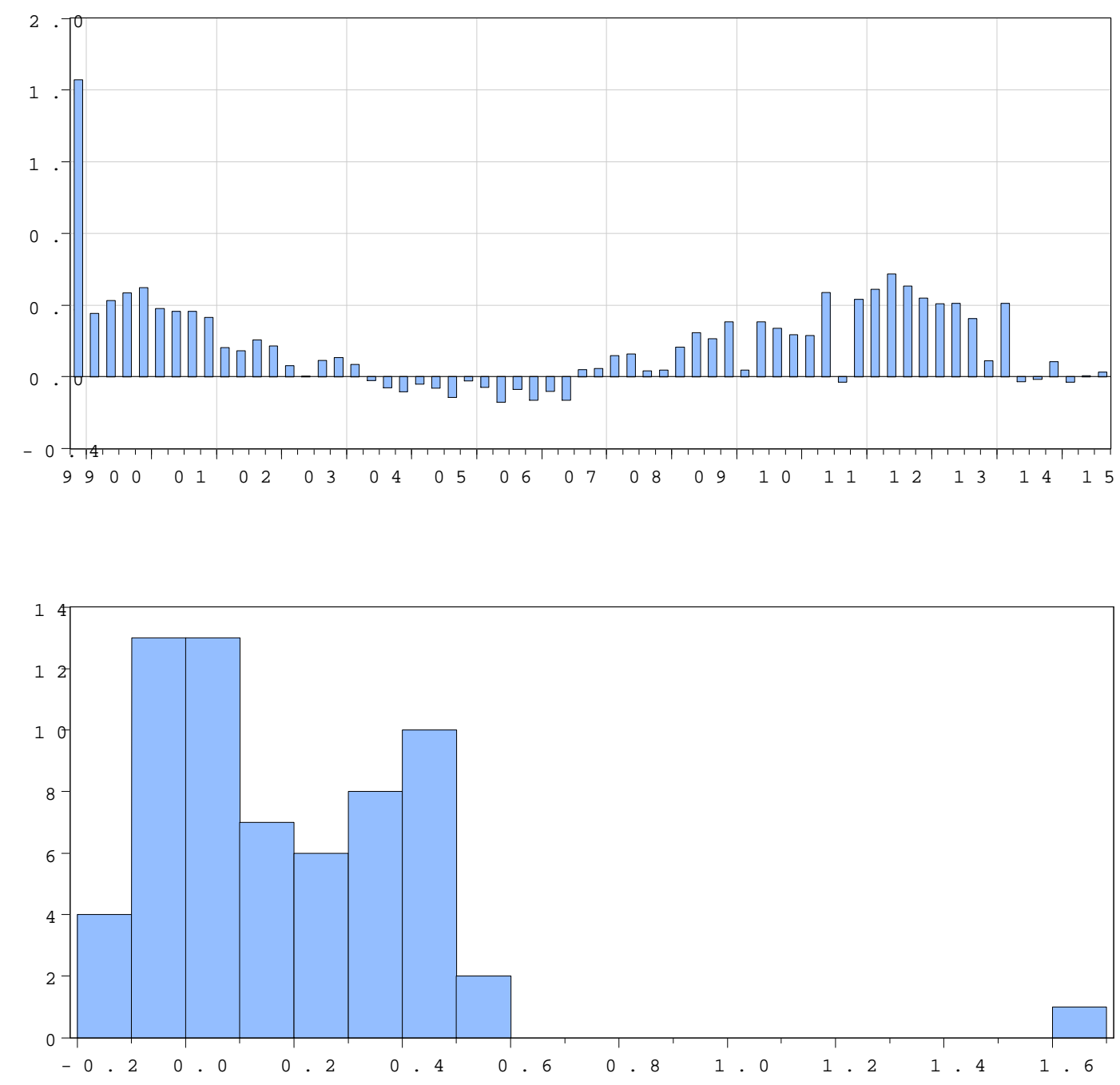

Figure 10: EA - Recursively Re-estimated Slope $(\kappa)$ of the TVT-NKPC at Horizon of 1 Quarter (MOE RMC proxy, fixed rolling window of 113 quarters), plot (top graph) and histogram (bottom graph); 1999Q4-2015Q3, 64 observations; mean 0.185, median 0.124, max 1.656, min -0.141, SD 0.273 

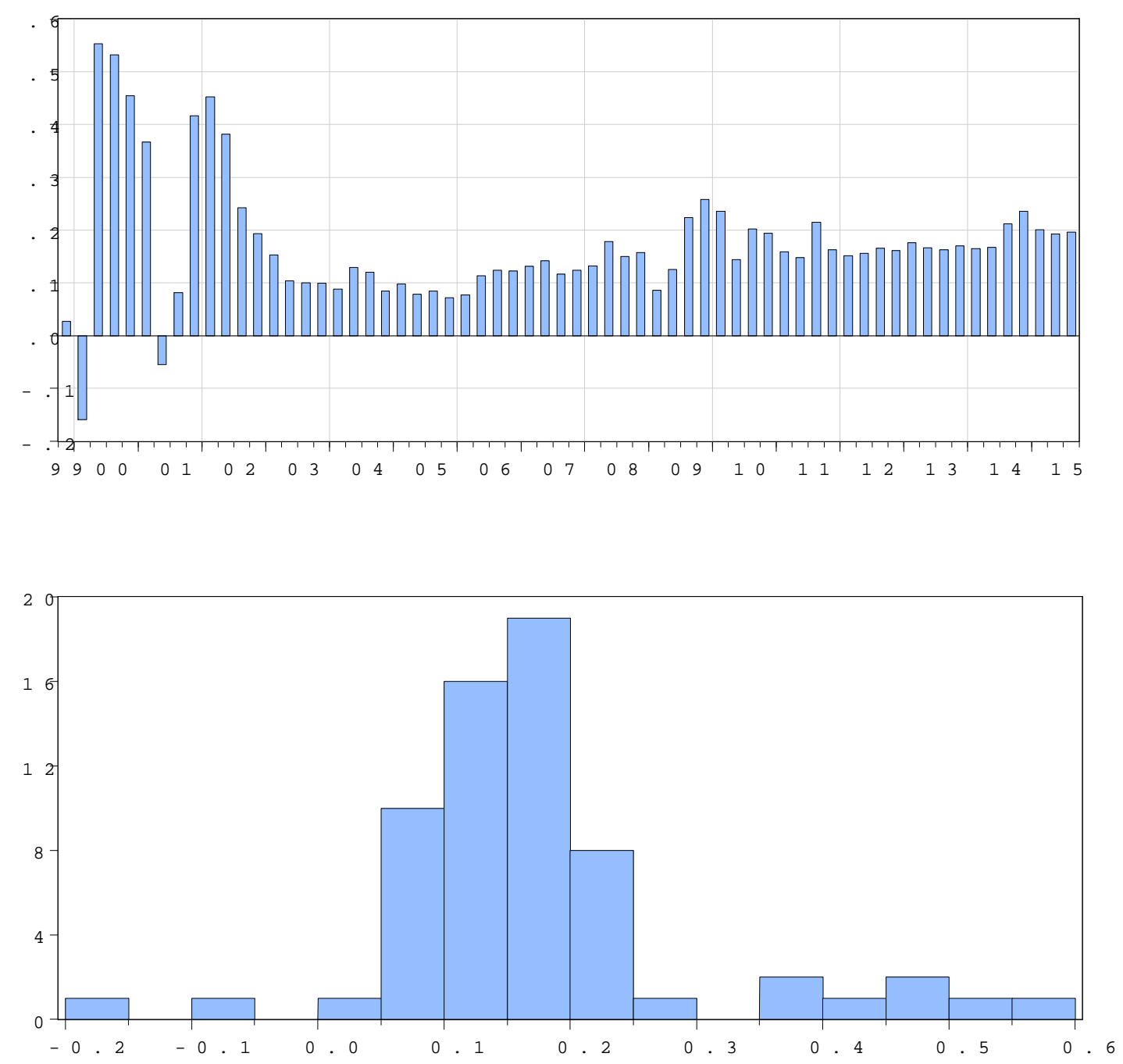

Figure 11: US - Recursively Re-estimated Slope $(\kappa)$ of the TVT-NKPC at Horizon of 1 Quarter (MOE RMC proxy, fixed rolling window of 113 quarters), plot (top graph) and histogram (bottom graph); 1999Q4-2015Q3, 64 observations; mean 0.173, median 0.157, max 0.553, min -0.159, SD 0.120 

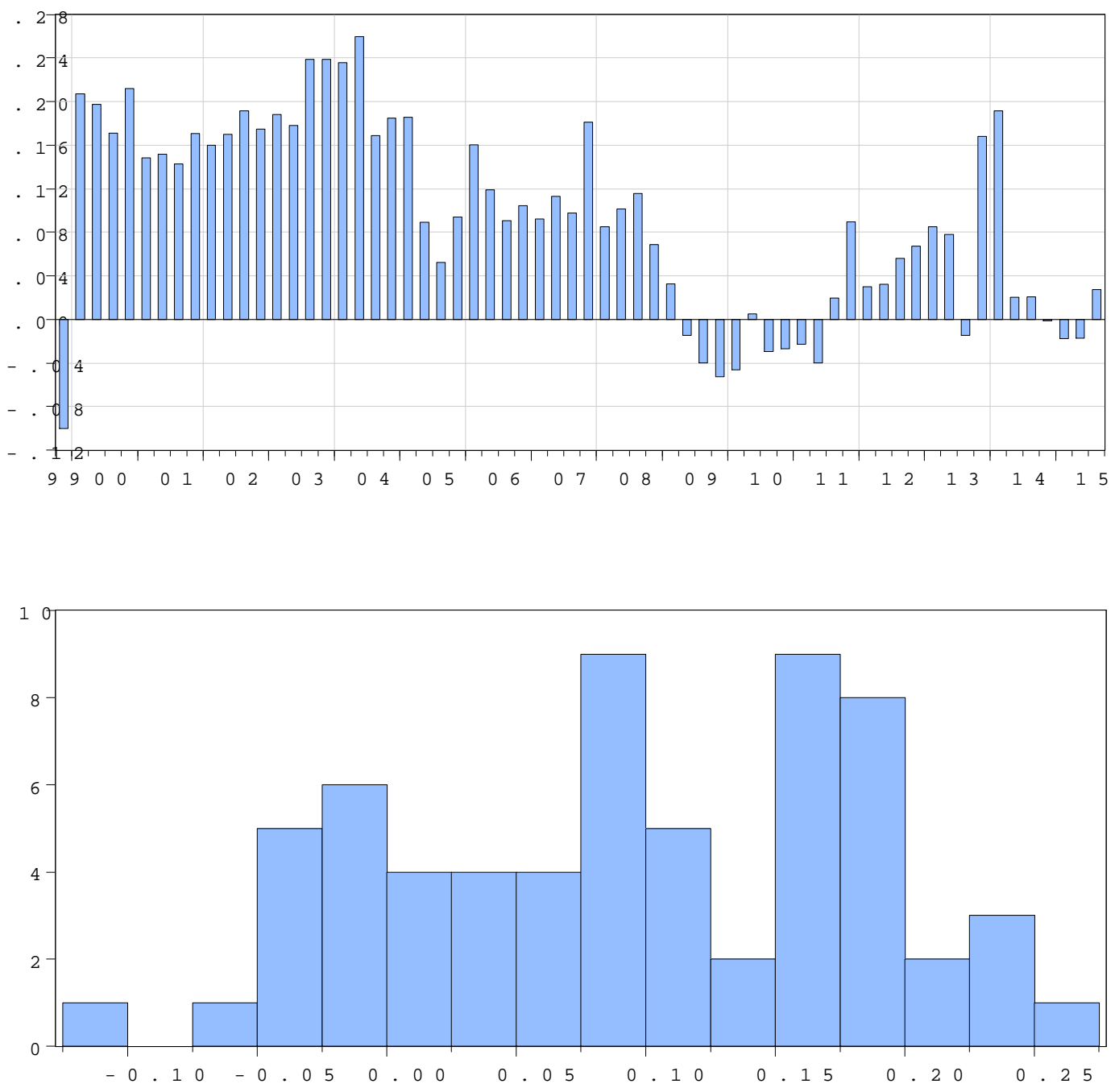

Figure 12: EA - Recursively Re-estimated Slope $(\kappa)$ of the TVT-NKPC at Horizon of 1 Quarter (RULC RMC proxy, fixed rolling window of 113 quarters), plot (top graph) and histogram (bottom graph); 1999Q4-2015Q3, 64 observations; mean 0.094, median 0.093, max 0.260, min -0.100, SD 0.089 

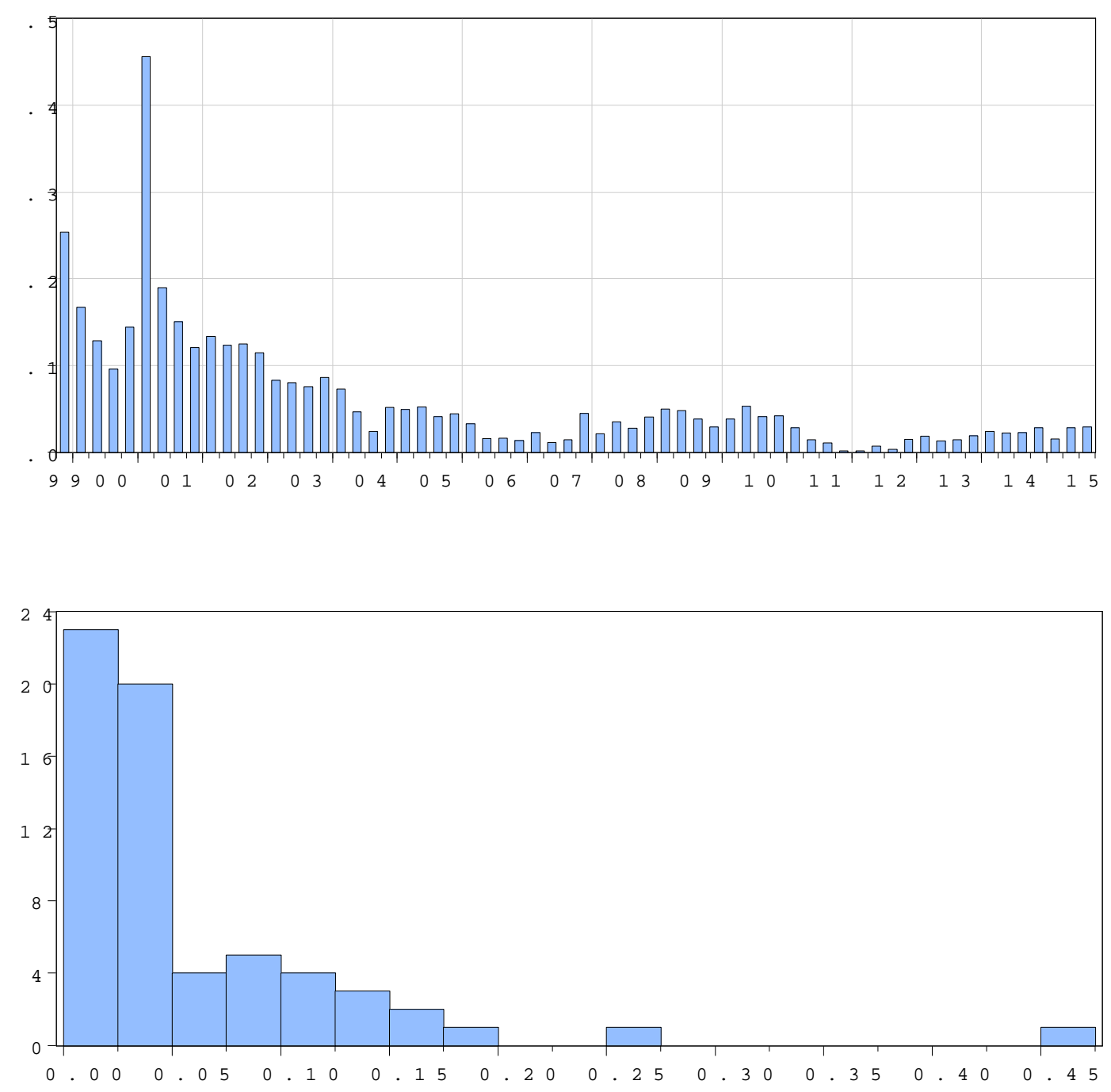

Figure 13: US - Recursively Re-estimated Slope $(\kappa)$ of the TVT-NKPC at Horizon of 1 Quarter (RULC RMC proxy, fixed rolling window of 113 quarters), plot (top graph) and histogram (bottom graph); 1999Q4-2015Q3, 64 observations; mean 0.060, median 0.038, max 0.456, min $0.001, \mathrm{SD} 0.072$ 

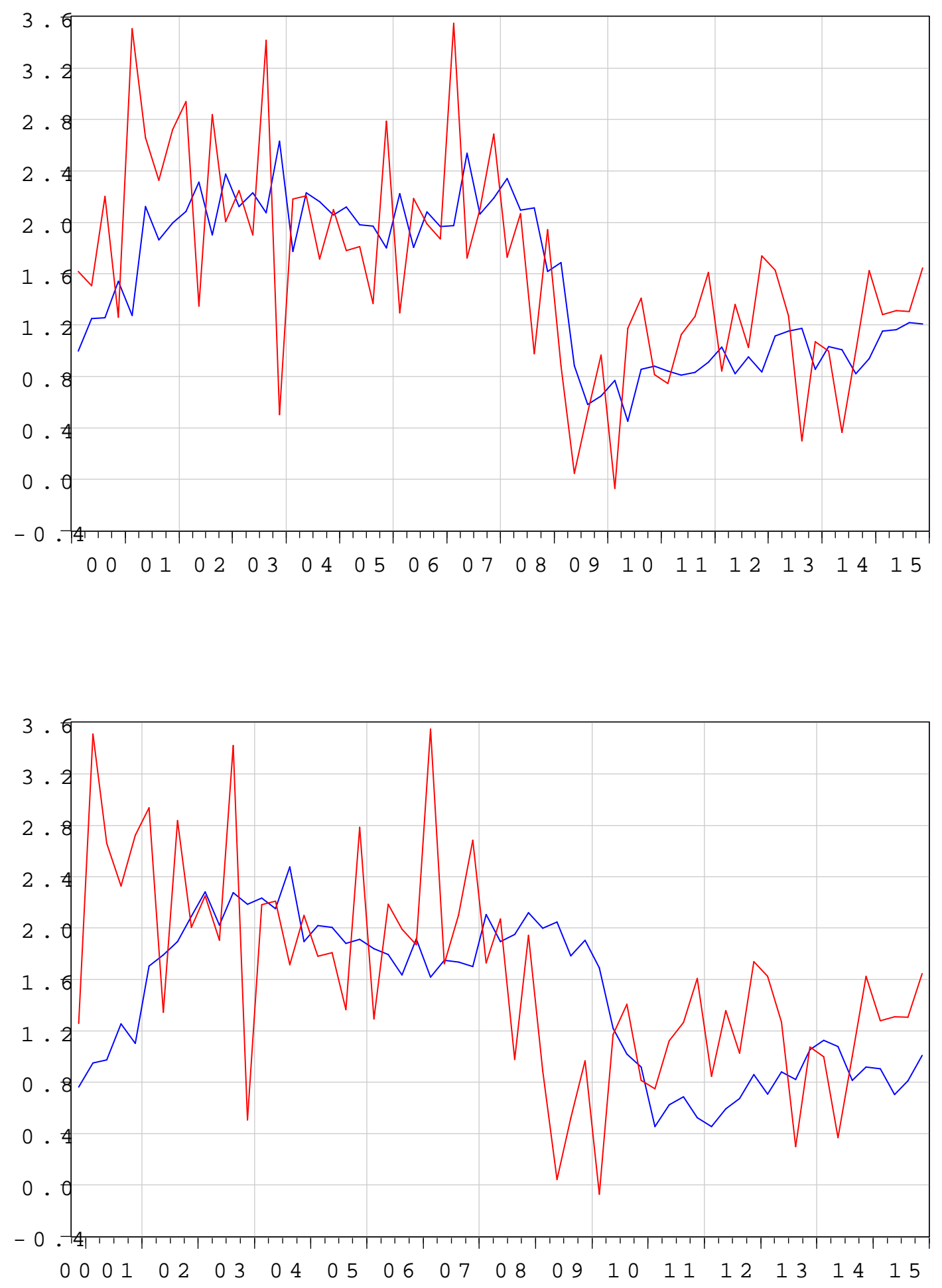

Figure 14: EA - Best TVT-NKPC forecasts (blue) against actual inflation (red) in the short run (as indicated by the bold fonts in Table 2 in the main text and Table 2 in this Appendix C): top graph -1 quarter ahead; bottom graph -4 quarters ahead; $\%$ per annum at annualized rate 

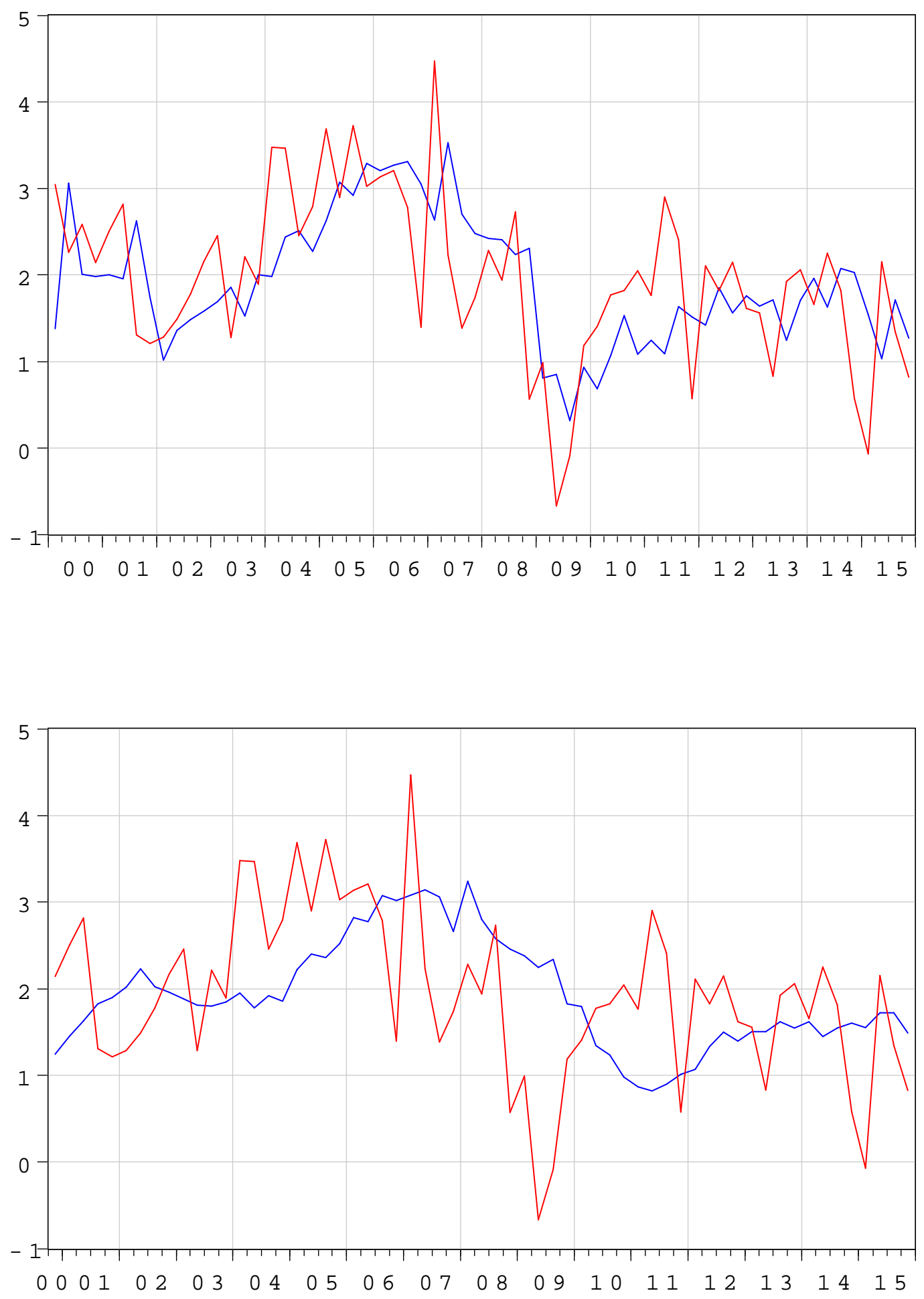

Figure 15: US - Best TVT-NKPC forecasts (blue) against actual inflation (red) in the short run (as indicated by the bold fonts in Table 3 in the main text and Table 3 in this Appendix C): top graph -1 quarter ahead; bottom graph -4 quarters ahead; $\%$ per annum at annualized rate 

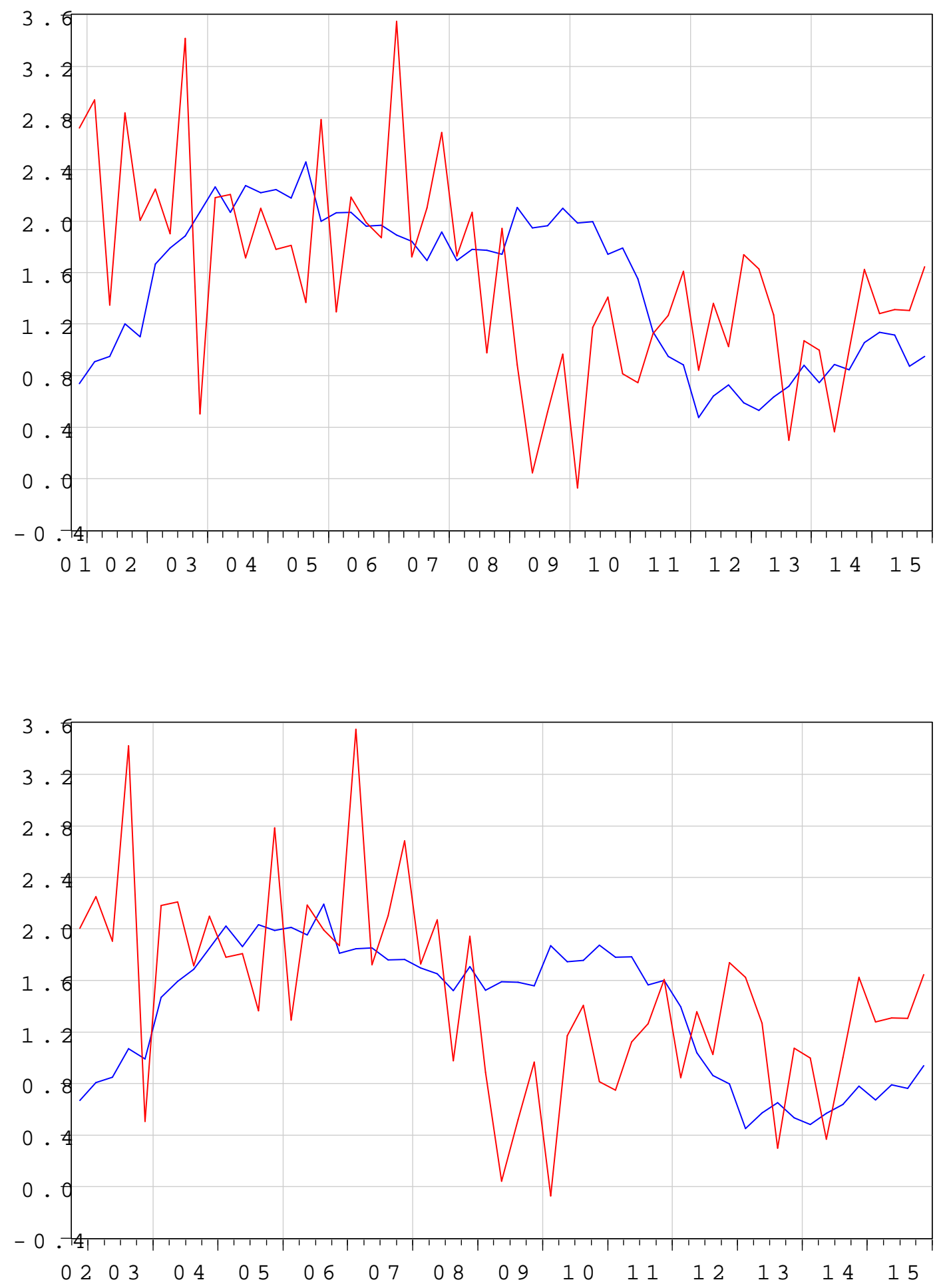

Figure 16: EA - Best TVT-NKPC forecasts (blue) against actual inflation (red) in the medium run (as indicated by the bold fonts in Table 2 in the main text and Table 2 in this Appendix C): top graph -8 quarters ahead; bottom graph - 12 quarters ahead; $\%$ per annum at annualized rate 

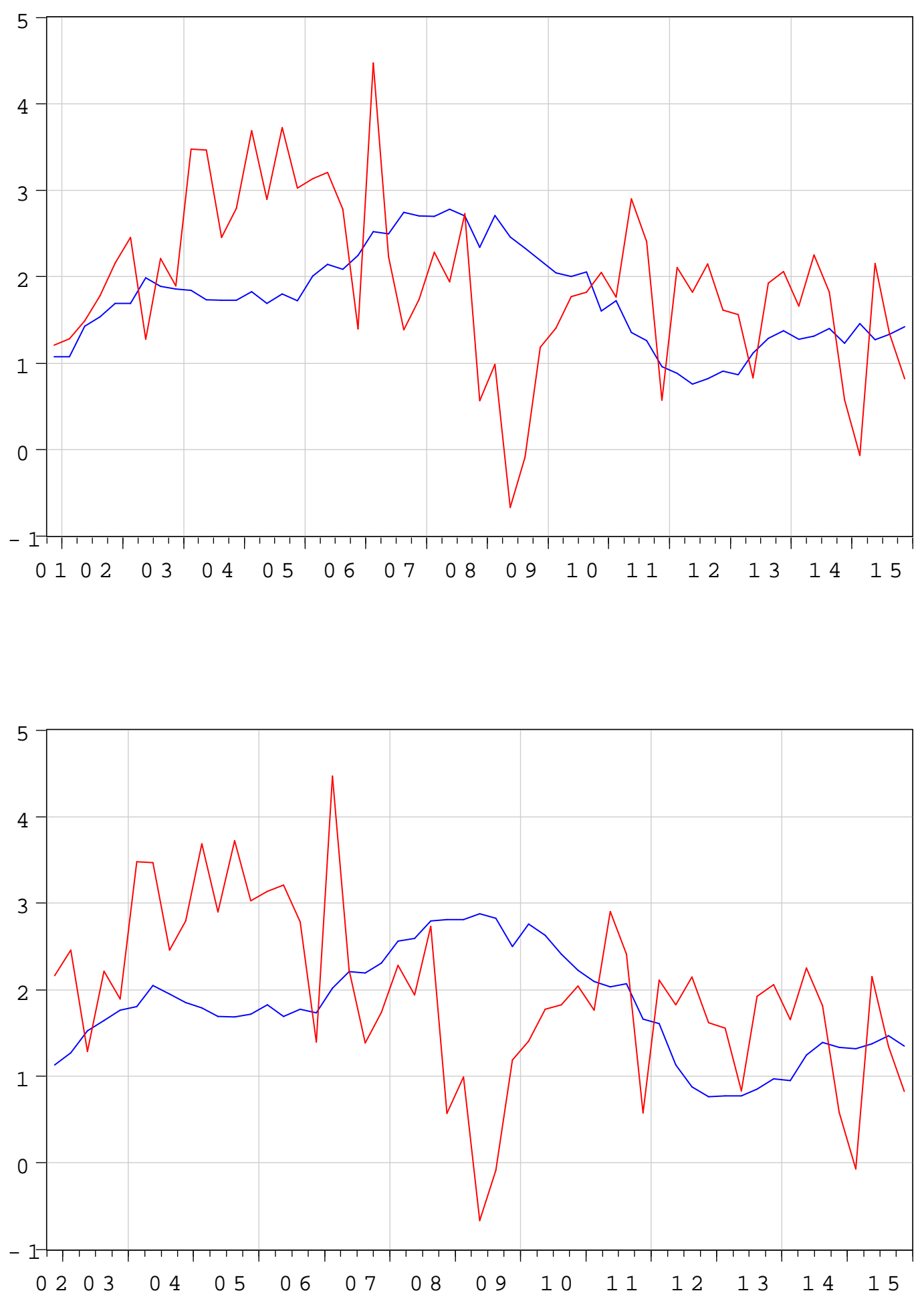

Figure 17: US - Best TVT-NKPC forecasts (blue) against actual inflation (red) in the medium run (as indicated by the bold fonts in Table 3 in the main text and Table 3 in this Appendix C): top graph -8 quarters ahead; bottom graph -12 quarters ahead; $\%$ per annum at annualized rate 

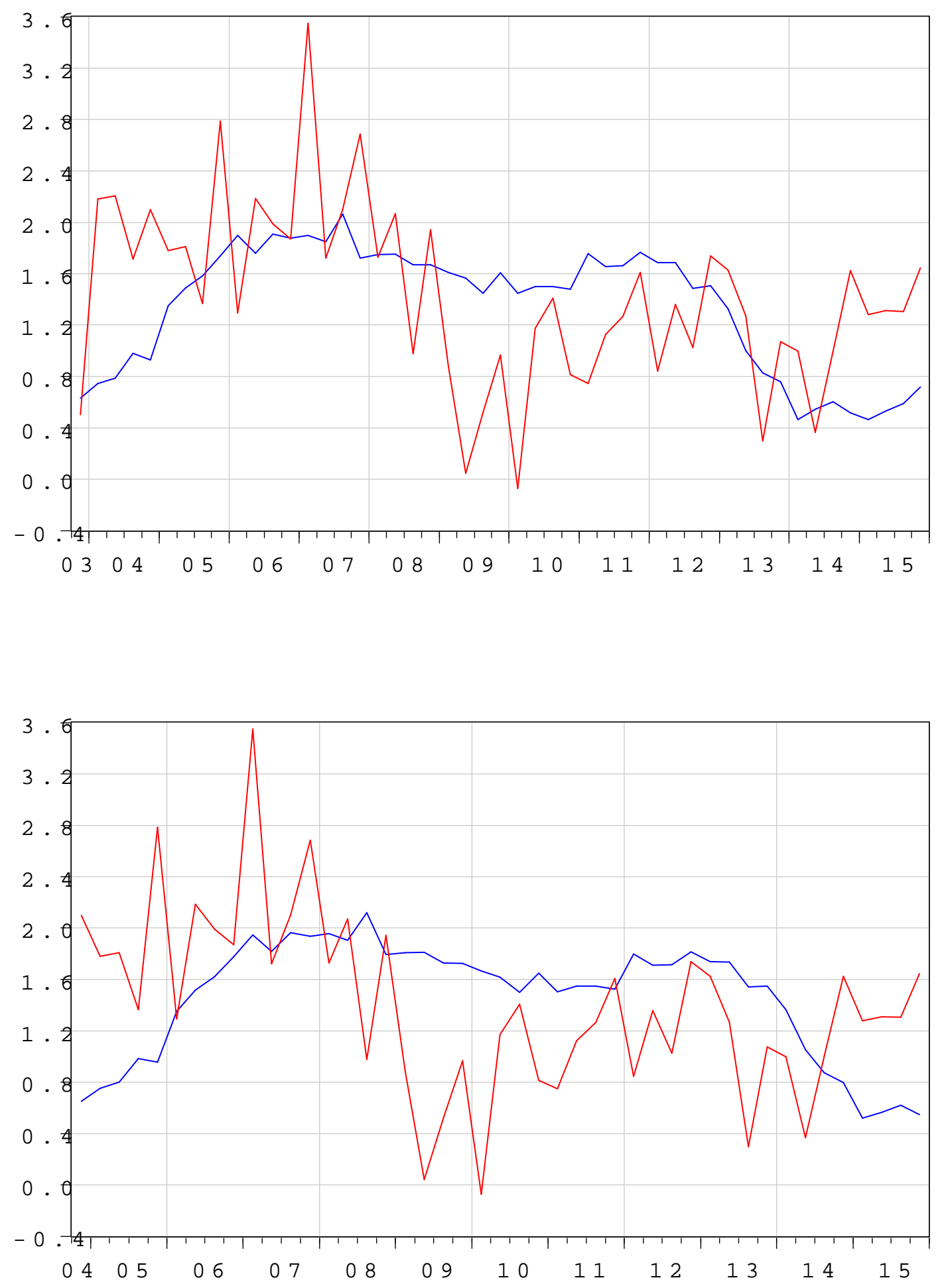

Figure 18: EA - Best TVT-NKPC forecasts (blue) against actual inflation (red) in the long run (as indicated by the bold fonts in Table 2 in the main text and Table 2 in this Appendix C): top graph - 16 quarters ahead; bottom graph - 20 quarters ahead; \% per annum at annualized rate 

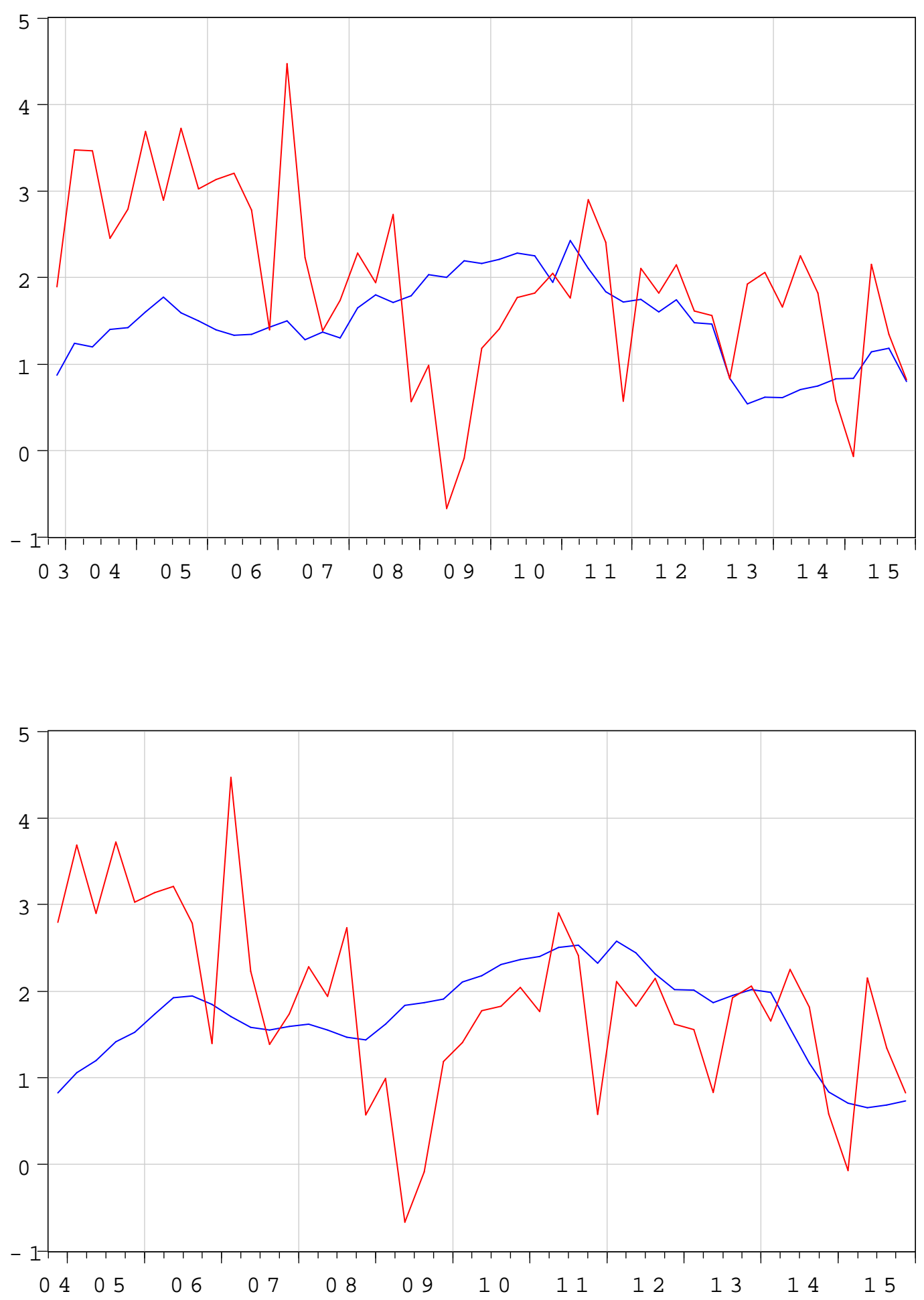

Figure 19: US - Best TVT-NKPC forecasts (blue) against actual inflation (red) in the long run (as indicated by the bold fonts in Table 3 in the main text and Table 3 in this Appendix C): top graph - 16 quarters ahead; bottom graph - 20 quarters ahead; $\%$ per annum at annualized rate 